\title{
Synthesis of Quinolinone Alkaloids via Aryne Insertions into Unsymmetric Imides in Flow
}

\author{
Johannes Schwan, Merlin Kleoff, Bence Hartmayer, Philipp Heretsch,* and Mathias Christmann*
}

Freie Universität Berlin, Institut für Chemie und Biochemie, Takustr. 3, 14195 Berlin, Germany

Supporting Information Placeholder

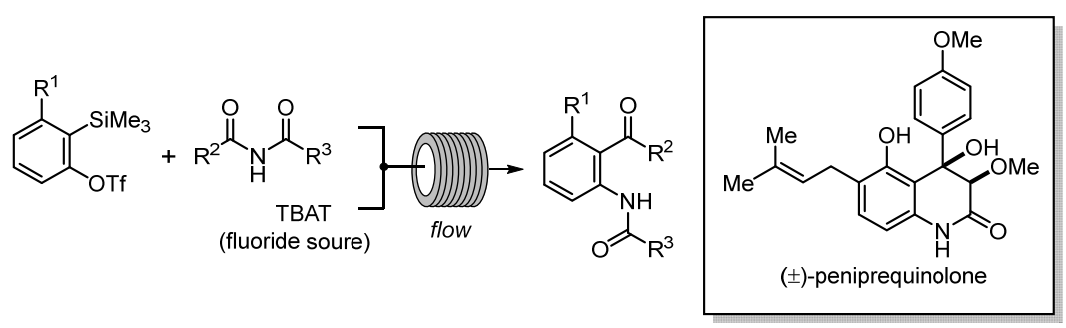

ABSTRACT: A general strategy for the synthesis of 3,4-dioxygenated quinolin-2-one natural products is reported. The key step is a regioselective insertion of arynes into unsymmetric imides. When performed in continuous flow, the reaction proceeded within minutes. The resulting $N$-acylated 2 -aminobenzophenones were transformed to ( \pm )-peniprequinolone, $( \pm)$-aflaquinolones E and F, ( \pm )-6-deoxyaflaquinolone E, $( \pm)$-quinolinones $A$ and $B$, and $( \pm)$-aniduquinolone $\mathrm{C}$ in $1-3$ steps.

The growing family of 3,4-dioxygenated quinolin-2-ones constitutes a valuable source of molecules with cytotoxic, nematicidal and antiviral activities. ${ }^{1,2}$ Biosynthetically, these fungal secondary metabolites are derived from the nonribosomal peptide synthetase (NRPS) product cyclopeptin (1), an adduct of anthranilic acid and either $O$-methyl-L-tyrosine ${ }^{3}$ or phenylalanine ${ }^{4}$ (Scheme 1a). Groll and Hintermann demonstrated that a single enzyme, AsqJ, catalyzes a multistep transformation of the benzodiazepine core of $\mathbf{1}$ into the parent quinolone $2 .{ }^{5}$ Downstream processing into the final natural products may involve prenylation $(\mathrm{n}=0)$, double prenylation $(\mathrm{n}=1)$ as well as additional oxidation steps. ${ }^{6}$ Following this synthetic blueprint, Nature has evolved a "library" of bioactive secondary metabolites ${ }^{3,7}$ from which only yaequinolone $\mathrm{J} 1, \mathrm{~J} 2$, and $\mathrm{A}_{2}$ have been synthesized to date (Scheme $1 \mathrm{~b}$ ). ${ }^{8}$ Since these natural products share a common heterocyclic core, we decided to develop a step-efficient, ${ }^{9}$ modular approach to access the entire group of these alkaloids (Scheme 1c). Synthetically, the 3,4-dioxygenated quinolin-2-one core (6) can be obtained by intramolecular glycolate aldolizations. ${ }^{10}$ We speculated that aryne chemistry ${ }^{11}$ could provide a straightforward access to $N$-glycolated 2-aminobenzophenones (5) and thus enable the synthesis of this natural product family. Based on the contributions of Greaney11a (acyclated carbamates), Stoltz ${ }^{11 \mathrm{~b}}$ (symmetric imides), and $\mathrm{Xu}^{11 \mathrm{~d}}$ (acrylamides), we speculated that a selective $\sigma-\mathrm{C}-\mathrm{N}$ insertion of in-situ generate arynes (3) into unsymmetric imides 4 could deliver $\mathbf{5}$ in a one-pot operation ${ }^{12}$ (Scheme 2). With these substrates, the challenge lies in the differentiation of two similar $N$-acyl groups.
Scheme 1. Outline of the Work
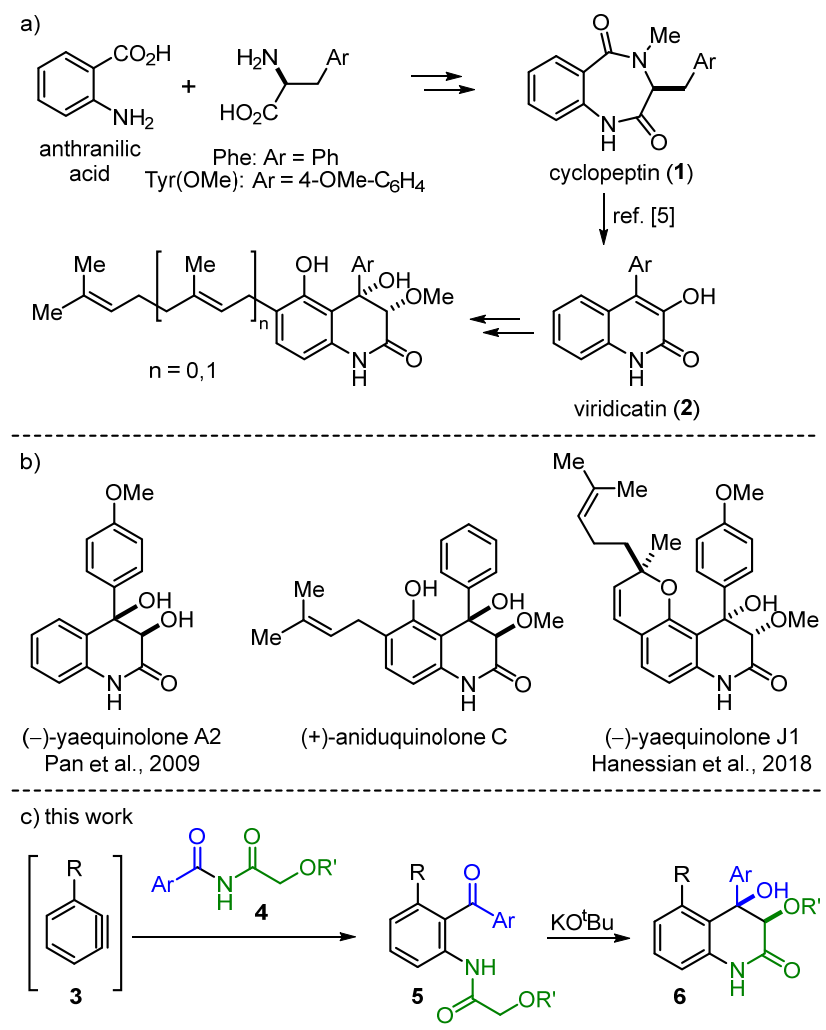
As a starting point, we investigated the insertion of benzyne precursor $\mathbf{3} \mathbf{a}^{13}$ into the unsymmetric imide $\mathbf{4 a}$. Under the conditions reported by Stoltz et al., ${ }^{11 \mathrm{~b}}$ benzophenone $\mathbf{5 a}$ was formed in $16 \%$ yield with only a slight preference for the $\mathbf{5 a}$ over its constitutional isomer $\mathbf{7}$ (Table 1, entry 1). A screening of solvents revealed that acetonitrile gave the best selectivity of 5.7:1 (entry 3). Among different fluoride sources, cesium fluoride gave the highest yield of $\mathbf{5 a}$ with $33 \%$ and an isomeric ration of 3.7:1 (entry 4). Upon extended reaction time, 7 undergoes slow thermal decomposition leading to a slightly increased isomer in favor of $\mathbf{5 a}$. Furthermore, it turned out that also imide 4a slowly decomposes to benzamide at elevated temperatures and under basic conditions.

Due to superior heat and mass transfer, reactions performed in flow can significantly be accelerated. Therefore, intermediates such as $\mathbf{8}$ often react with higher selectivity and yield, enabling reactions that are otherwise impossible. ${ }^{14}$ Surprisingly, only few examples are reported, where arynes are generated and used in a flow reactor. ${ }^{15}$

\section{Table 1. Reaction Development in Batch ${ }^{\mathrm{a}}$}

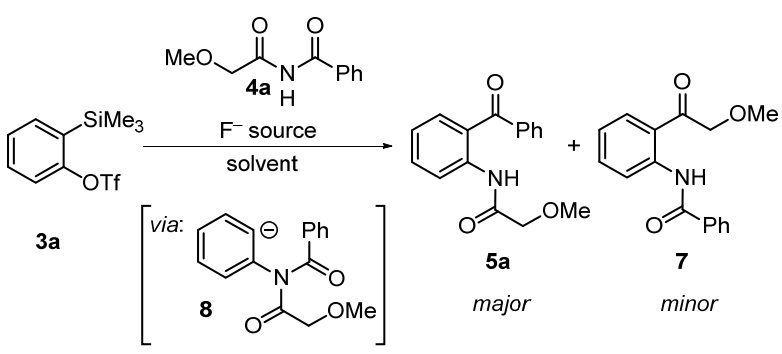

$\begin{array}{ccccc}\text { Entry } & \text { Solvent } & \mathbf{F}^{-} \text {Source } & \mathbf{5 a : 7 b} & \mathbf{5 a}(\mathbf{0}) \mathbf{c} \\ 1 & \text { PhMe } & \text { TBAT } & 1.3: 1 & 16 \\ 2 & \text { THF } & \text { TBAT } & 2.0: 1 & 18 \\ 3 & \text { MeCN } & \text { TBAT } & 5.7: 1 & 25 \\ 4^{\mathrm{d}} & \text { MeCN } & \text { CsF } & 3.7: 1 & 33 \\ 5^{\mathrm{d}, \mathrm{e}} & \mathrm{MeCN} & \mathrm{KF} & 4.0: 1 & 20\end{array}$

aReaction conditions: 4 a $(0.26 \mathrm{mmol}, 1$ equiv. $)$, 3a ( $0.39 \mathrm{mmol}, 1.5$ equiv.), $\mathrm{F}^{-}$source ( $0.52 \mathrm{mmol}, 2$ equiv.), solvent $(2.0 \mathrm{~mL}), 60^{\circ} \mathrm{C}, 16 \mathrm{~h}$; bratios were determined by ${ }^{1} \mathrm{H}$ NMR integration; cisolated yield; ${ }^{\mathrm{d}}$ conducted at $80^{\circ} \mathrm{C}$ for $4 \mathrm{~h}$; e18-crown6 (1 equiv.) and $4 \AA$ mol sieves were used as additives.

In order to suppress thermal imide decomposition by shortening the reaction time, a flow protocol seemed promising. Due to its high solubility, tetrabutylammonium difluorotriphenylsilicate (TBAT) is a better fluoride source for flow reactions than cesium fluoride. An optimization of reaction time, temperature and stoichiometry (see SI, for details), increased the yield of $\mathbf{5 a}$ from $25 \%$ in batch (Table 1 , entry 3), to $52 \%$ when conducted at $65^{\circ} \mathrm{C}$ with 1.5 equiv. of $3 \mathbf{a}$ at a flow rate of $1 \mathrm{~mL} / \mathrm{min}$ (Table 2, entry 1). Under these conditions, a regioselectivity of 2.9:1 (5a:7) was observed, while the residence time was reduced to 4 minutes.

Using the optimized conditions, a collection of substituted arynes and imides were reacted. According to the proposed mechanism, the aryne insertion proceeds via intermediacy of $8 .{ }^{11 a, b, 16}$ Greaney et al. ${ }^{11 a}$ showed that a major byproduct results from premature protonation of $\mathbf{8}$. Remarkably, under flow conditions even the use of wet acetonitrile did not increase the amount of byproduct, thus rendering the flow process more sustainable. As arynes bearing a 3-alkoxy substituent have been reported undergo regioselective nucleophilic attack in the meta-position, ${ }^{17}$ we expected the resulting benzophenones to provide us with an access to the 5hydroxyquinolinone cores present in aflaquinolone $\mathrm{E}(\mathbf{9})^{18}$ and quinolinone B (10). ${ }^{19 \mathrm{c}}$ In agreement with the literature reports, ${ }^{11 \mathrm{a}, \mathrm{b}, 20}$ we too observed significantly lower yields with those substrates. Thus, when using 3-O-benzyl and 3$O$-allyl substituted aryne precursors $\mathbf{3 b}$ and $\mathbf{3 c}$, the yield dropped to $18 \%$ to $30 \%$ (Table 2, entry 3-6). The insertion was also performed with 4-bromo substituted imide $4 \mathbf{c}$ to access an unnatural quinolinone derivative with a handle for cross coupling chemistry as well as $O$-benzyl substituted imide $\mathbf{4 d}$ to access aflaquinolone $\mathrm{F}(\mathbf{1 1}) \cdot{ }^{18}$ In all cases, benzophenones $\mathbf{5 a - h}$ were obtained as the major product along with unreacted imide 4a-d.

Table 2. Aryne Insertion in Flow

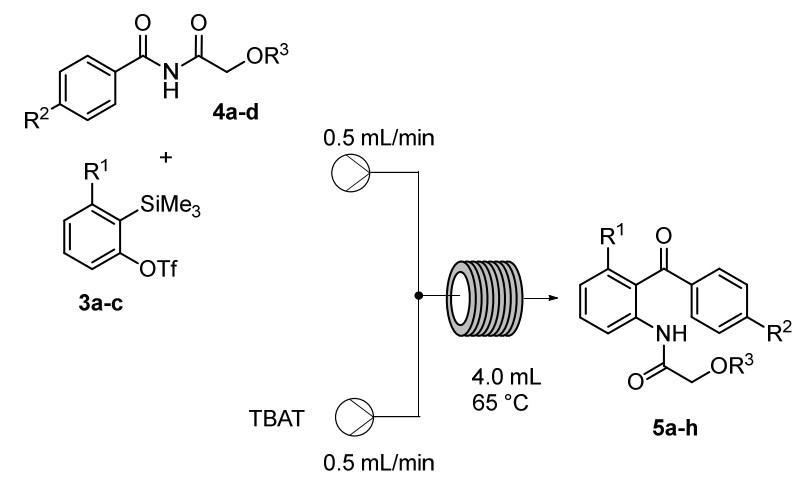

\begin{tabular}{cccccc} 
Entry & $\begin{array}{c}\text { Compound } \\
\mathbf{5}\end{array}$ & $\mathbf{R}^{\mathbf{1}}$ & $\mathbf{R}^{\mathbf{2}}$ & $\mathbf{R}^{\mathbf{3}}$ & $\mathbf{5}(\mathbf{\%})^{\mathbf{b}}$ \\
\hline 1 & $\mathbf{a}$ & $\mathrm{H}$ & $\mathrm{H}$ & $\mathrm{Me}$ & 52 \\
$2^{\mathrm{c}}$ & $\mathbf{b}$ & $\mathrm{H}$ & $\mathrm{OMe}$ & $\mathrm{Me}$ & 60 \\
3 & $\mathbf{c}$ & $\mathrm{H}$ & $\mathrm{Br}$ & $\mathrm{Me}$ & 23 \\
4 & $\mathbf{d}$ & $\mathrm{OBn}$ & $\mathrm{H}$ & $\mathrm{Me}$ & 35 \\
$5^{\mathrm{c}}$ & $\mathbf{e}$ & $\mathrm{OBn}$ & $\mathrm{OMe}$ & $\mathrm{Me}$ & 24 \\
6 & $\mathbf{f}$ & $\mathrm{H}$ & $\mathrm{H}$ & $\mathrm{Bn}$ & 40 \\
7 & $\mathbf{g}$ & OAllyl & $\mathrm{H}$ & $\mathrm{Me}$ & 18 \\
$8^{\mathrm{c}}$ & $\mathbf{h}$ & OAllyl & OMe & $\mathrm{Me}$ & 30
\end{tabular}

aReaction conditions: 4 ( $0.20 \mathrm{mmol}, 1$ equiv., $0.1 \mathrm{M}$ in $\mathrm{MeCN}$ ), 3 ( $0.30 \mathrm{mmol}, 1.5$ equiv., $0.15 \mathrm{~m}$ in $\mathrm{MeCN}$ ), TBAT ( $0.36 \mathrm{mmol}$, 1.8 equiv., $0.18 \mathrm{M}$ in $\mathrm{MeCN}), 4 \mathrm{~mL}$ reactor volume, $65^{\circ} \mathrm{C}$; bisolated yield; $\mathrm{c} \mathbf{4 b}(\mathrm{c}=0.01 \mathrm{M}$ in $\mathrm{MeCN}), \mathbf{3 a}-\mathbf{c}(\mathrm{c}=0.015 \mathrm{M}$ in $\mathrm{MeCN})$, TBAT ( $\mathrm{c}=0.018 \mathrm{M}$ in $\mathrm{MeCN})$.

With the benzophenones $\mathbf{5 a - h}$ in hand, we next investigated an intramolecular reaction ${ }^{10}$ to forge the 3,4-dioxygenated quinoline-2-one core. Using an excess of potassium tert-butoxide in tetrahydrofuran at $0{ }^{\circ} \mathrm{C}$, the quinolinones 6a-g were obtained as single diastereomers (Scheme 2). 
Following this procedure, the natural products $( \pm)$-6-deoxyaflaquinolone E (6a) ${ }^{21}$ and $( \pm)$-quinolinone $A(\mathbf{6 b})^{20 c}$ were prepared in $91 \%$ and $84 \%$ yield, respectively.

\section{Scheme 2. Intramolecular Aldolization}<smiles>[R16]c1cccc(Cc2c([R])ccc(CCO)c2NC(=O)CO)c1</smiles><smiles></smiles><smiles>CC(O)C1(c2ccccc2)C(=O)Nc2ccccc21</smiles>

6-deoxyaflaquinolone (6a, 91\%)<smiles>COc1ccc(C2(O)c3ccccc3NC(=O)C2OC)cc1</smiles><smiles>CCC1(c2ccc(Br)cc2)C(=O)Nc2ccccc21</smiles>

quinolinone A 4'-bromoquinolinone A (6c, 90\%)<smiles>[R]c1cccc2c1C(O)(c1ccccc1)C(OC)C(=O)N2</smiles><smiles>[R]c1cccc2c1C(O)(c1ccc(OC)cc1)C(OC)C(=O)N2</smiles><smiles>[R10]C1C(=O)Nc2ccccc2C1(O)c1ccccc1</smiles>

6d: $\mathrm{R}^{1}=\mathrm{OBn}$ 9: $\mathrm{R}^{1}=\mathrm{OH} \& \mathrm{H}_{2}, \mathrm{Pd} / \mathrm{C}$ aflaquinolone $\mathrm{E}$ ( $71 \%, 2$ steps)<smiles>C=CCOc1cccc2c1C(O)(c1ccccc1)C(OC)C(=O)N2</smiles>

$6 \mathbf{g}(67 \%)$<smiles>[R]CC(C)(O)c1ccc([R])cc1</smiles>

6g: $\mathrm{R}^{2}=\mathrm{H}$ 6h: $\mathrm{R}^{2}=\mathrm{OMe}$<smiles>[R2]c1ccc(C2(O)c3c(ccc(CC=C)c3O)NC(=O)C2OC)cc1</smiles>

12a: $R^{2}=H(71 \%)$

12b: $R^{2}=$ OMe $(63 \%)$

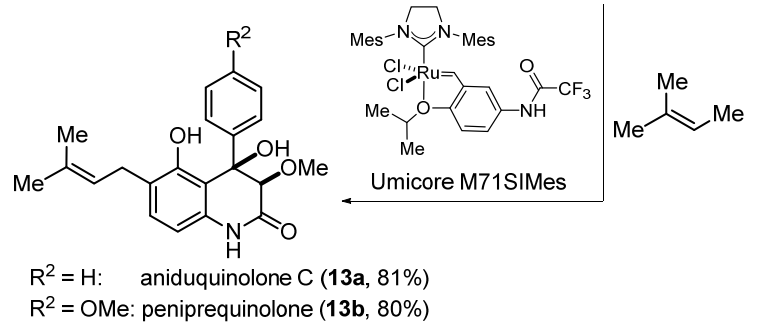

In conclusion, we have devised a general approach for the synthesis of 3,4-dioxygenated quinolin-2-one natural products. The sequence proceeds through an insertion of arynes into unsymmetric imides followed by a diastereoselective intramolecular aldolization. A flow protocol for the aryne insertion was developed giving access to $\mathrm{N}$-glycolated 2aminobenzophenones within minutes. By this not immediately evident, yet powerful disconnection, the quinolinone natural products peniprequinolone, aflaquinolone $\mathrm{E}, \mathrm{F}$, quinolone $\mathrm{A}, \mathrm{B}$, and aniduquinolone $\mathrm{C}$ were synthesized in three to six steps. ${ }^{23}$ Future work will focus on the synthesis of quinolinone natural products with other side chains and higher oxidation levels.

\section{ASSOCIATED CONTENT}

\section{Supporting Information}

The Supporting Information is available free of charge on the ACS Publications website.

\section{AUTHOR INFORMATION}

\section{Corresponding Authors}

*E-mail: mathias.christmann@fu-berlin.de

* E-mail: philipp.heretsch@fu-berlin.de

\section{Notes}

The authors declare no competing financial interest.

\section{ACKNOWLEDGMENT}

We thank Florian Bartels and Reinhold Zimmer (FU Berlin) for helpful discussions. We also thank Umicore for the generous donation of metathesis catalysts.

\section{REFERENCES}


(1) For a review of the natural product class, see: Simonetti, S. O.; Larghi, E. L.; Kaufman, T. S. Nat. Prod. Rep. 2016, 33, 14251446.

(2) For recent examples, see: (a) Shao, C.; Wang, C.; Xu, R.; Guan, F.; Wei, M. U.S. Pat. Appl. Publ. 2018, US20180028524A1. (b) Shao, C.; Wang, C.; Mu, X.; Xu, R. U.S. Pat. Appl. Publ. 2018, US20180028523A1. (c) Kempter, C.; Roos, U.; Schorderet Weber, S.; Ebinger, Y.; Glaser, S. U.S. Pat. Appl. Publ. 2014, US8648091B2. (d) Gauvry, N.; Kempter, C.; Pautrat, F.; Roos, U. PCT Int. Appl. 2014, W02014044615A1. (e) Meiring, L.; Petzer, J. P.; Petzer, A. Mini-Reviews in Medicinal Chemistry 2018, 18, 828836. (f) Sridharan, V.; Suryavanshi, P. A.; Menéndez, J. C. Chem. Rev. 2011, 111, 7157-7259. (g) Khan, K. Molecules 2016, 21, 268.

(3) Scherlach, K.; Hertweck, C. Org. Biomol. Chem. 2006, 4, 35173520.

(4) (a) Walsh, C. T.; Haynes, S. W.; Ames, B. D.; Gao, X.; Tang, Y. ACS Chem. Biol. 2013, 8, 1366-1382. (b) Luckner, M.; Winter, K.; Reisch, J. Eur. J. Biochem. 1969, 7, 380-384.

(5) (a) Bräuer, A.; Beck, P.; Hintermann, L.; Groll, M. Angew. Chem. Int. Ed. 2016, 55, 422-426. (b) Mader, S. L.; Bräuer, A.; Groll, M.; Kaila, V. R. I. Nat. Chem. 2018, 9, 1168.

(6) Zou, Y.; Zhan, Z.; Li, D.; Tang, M.; Cacho, R. A.; Watanabe, K.; Tang, Y. J. Am. Chem. Soc. 2015, 137, 4980-4983.

(7) Hertweck, C. Nat. Chem. Biol. 2009, 5, 450-452.

(8) (a) Li, X.; Huo, X.; Li, J.; She, X.; Pan, X. Chin. J. Chem. 2009, 27, 1379-1381. (b) Shashidhar, V. V.; Jakkepally, S.; Hanessian, S. Org. Lett. 2018, 20, 4277-4280.

(9) Schwan, J.; Christmann, M. Chem. Soc. Rev. 2018, doi: 10.1039/C8CS00399H.

(10) Ueki, H.; Ellis, T. K.; Khan, M. A.; Soloshonok, V. A. Tetrahedron 2003, 59, 7301-7306.

(11) (a) Pintori, D. G.; Greaney, M. F. Org. Lett. 2010, 12, 168171. (b) Wright, A. C.; Haley, C. K.; Lapointe, G.; Stoltz, B. M. Org. Lett. 2016, 18, 2793-2795. (c) Dong, Y.; Liu, B.; Chen, P.; Liu, Q.; Wang, M. Angew. Chem. Int. Ed. 2014, 53, 3442-3446. (d) Wang, W.; Peng, X.; Qin, X.; Zhao, X.; Ma, C.; Tung, C.-H.; Xu, Z. J. Org. Chem. 2015, 80, 2835-2841. (e) Peng, X.; Jiang, C.; Sun, D.; Xu, Z.; Tung, C.-H. Org. Lett. 2014, 16, 5354-5357. (f) Reddy, R. S.; Lagishetti, C.; Kiran, I. N.; You, H,; He, Y. Org. Lett. 2016, 18, 3818-3821. (g) Tores-Ochoa, R. O.; Buyck, T.; Wang, Q.; Zhu, J. Angew. Chem. Int. Ed. 2018, 57, 5679-5683. (h) Gampe, C. M.; Carreira, E. M. Angew. Chem. Int. Ed. 2012, 51, 3766-3778. (i) Pintori, D. G.; Greaney, M. F. Org. Lett. 2010, 12, 168-171.

(12) (a) Vaxelaire, C.; Winter, P.; Christmann, M. Angew. Chem. Int. Ed. 2011, 50, 3605-3607. (b) Hayashi, Y. Chem. Sci. 2016, 7, 866-880.

(13) Himeshima, Y.; Sonoda, T.; Kobayashi, H. Chem. Lett. 1983, 12, 1211-1214.

(14) for reviews, see: (a) Movsisyan, M.; Delbeke, E. I. P.; Berton, J. K. E. T.; Battilocchio, C.; Ley, S. V.; Stevens, C. V. Chem. Soc. Rev. 2016, 45, $4892-4928$ (b) Wegner, J.; Ceylan, S.; Kirschning, A. Adv. Synth. Catal. 2012, 354, 17-57. c) Yoshida, J.-i.; Kim, H.; Nagaki, A. J. Flow Chem. 2017, 7, 60-64. d) Plutschack, M. B.; Pieber, B.; Gilmore, K.; Seeberger, P. H. Chem. Rev. 2017, 117, 1179611893.

(15) (a) Khadra, A.; Organ, M. G. J. Flow Chem. 2016, 6, 293-296 (b) Nagaki, A.; Ichinari, D.; Yoshida, J.-i. J. Am. Chem. Soc. 2014, 136, 12245-12248. c) He, Z.; Jamison, T. F. Angew. Chem. Int. Ed. 2014, 53, 3353-3357. d) Browne, D. L.; Wright, S.; Deadman, B. J.; Dunnage, S.; Baxendale, I. R.; Turner, R. M.; Ley, S. V. Rapid Commun. Mass Spectrom. 2012, 26, 1999-2010.

(16) Wang, Y.; Yu, Z.-X. J. Org. Chem. 2018, 83, 5384-5391.

(17) (a) Cheong, P. H. Y.; Paton, R. S.; Bronner, S. M.; Im, G. Y. J.; Garg, N. K.; Houk, K. N. J. Am. Chem. Soc. 2010, 132, 1267-1269. (b) Liu, Z.; Larock, R. C. J. Org. Chem, 2006, 71, 3198-3209. (c)
Matsumoto, T.; Hosoya, T.; Katsuki, M.; Suzuki, K. Tetrahedron Lett. 1991, 32, 6735-6736.

(18) Neff, S. A.; Lee, S. U.; Asami, Y.; Ahn, J. S.; Oh, H.; Baltrusaitis, J.; Gloer, J. B.; Wicklow, D. T. J. Nat. Prod. 2012, 464-472.

(19) (a) Kusano, M.; Koshino, H.; Uzawa, J.; Fujioka, S.; Kawano, T.; Kimura, Y. Biosci. Biotechnol. Biochem. 2014, 64, 2559-2568. (b) He, J.; Lion, U.; Sattler, I.; Gollmick, F. A.; Grabley, S.; Cai, J.; Meiner, M.; Schünke, H.; Schaumann, K.; Dechert, U.; Krohn, M. J. Nat. Prod. 2005, 68, 1397-1399. (c) Hayashi, H.; Nakatani, T.; Inoue, Y.; Nkayama, M.; Nozaki, H. Biosci. Biotechnol. Biochem. 1997, 61, 914-916.

(20) (a) Yoshida, H.; Watanabe, M.; Morishita, T.; Ohshita, J.; Kunai, A. Chem. Commun. 2007, 1505-1507. (b) Yoshida, H.; Kishida, T.; Watanabe, M.; Ohshita, J. Chem. Commun. 2008, 59635965. (c) Kou, K. G. M.; Pflueger, J. J.; Kiho, T.; Morrill, L. C.; Fisher, E. L.; Clagg, K.; Lebold, T. P.; Kisunzu, J. K.; Sarpong, R. J. Am. Chem. Soc. 2018, 140, 8105.

(21) An, C.-Y.; Li, X.-M.; Luo, H.; Li, C.-S.; Wang, M.-H.; Xu, G.-M.; Wang, B.-G. J. Nat. Prod. 2013, 76, 1896-1901.

(22) Simonetti, S. 0.; Larghi, E. L.; Kaufman, T. S. Org. Biomol. Chem. 2016, 14, 2625-2636.

(23) All spectra of prepared natural products are in agreement with those reported in ref. (18), (19) and (21). 


\section{Synthesis of Quinolinone Alkaloids via Aryne Insertions into Unsymmetric Imides in Flow}

Johannes Schwan, Merlin Kleoff, Bence Hartmayer, Philipp Heretsch* and Mathias Christmann*

Freie Universität Berlin, Institut für Chemie und Biochemie, Takustr. 3, 14195 Berlin, Germany

\section{Supporting Information}

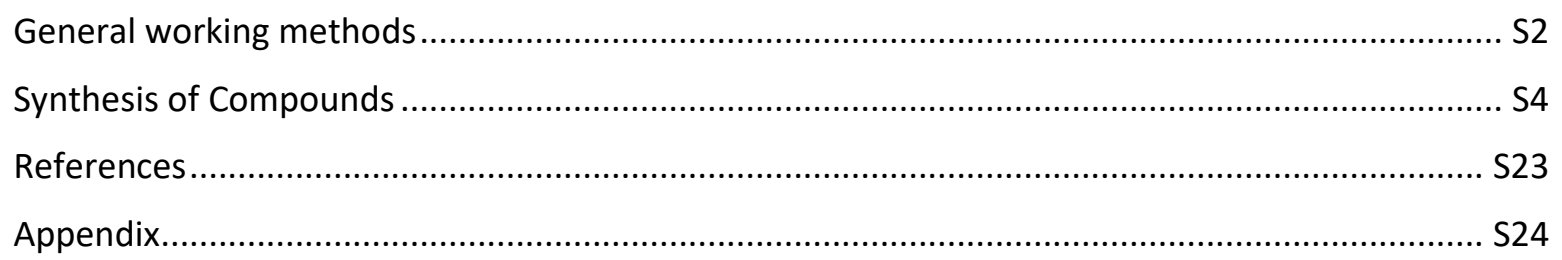




\section{General working methods}

The analytical data was obtained with the help of the following equipment.

\section{NMR spectroscopy}

${ }^{1} \mathrm{H}$ and ${ }^{13} \mathrm{C}$ NMR spectra were acquired on a JEOL ECX $400(400 \mathrm{MHz})$, JEOL ECP $500(500 \mathrm{MHz})$ and a Bruker Avance 700 (700 MHZ) in $\mathrm{CDCl}_{3}$ as a solvent. The chemical shifts were reported relative to $\mathrm{CDCl}_{3}\left(\delta={ }^{1} \mathrm{H}: 7.26 \mathrm{ppm},{ }^{13} \mathrm{C}: 77.16 \mathrm{ppm}\right)$. The multiplicities of the signals are described using the following abbreviations: $s=$ singlet, $d=$ doublet, $t=$ triplet, $q=$ quartet, $p=$ quintuplet, $b r=$ broad and combinations thereof.

The spectra were evaluated with the software MestRec.

Mass spectra were obtained on a ESI-FTICR-MS: Ionspec QFT-7 (Agilent/Varian), or a HR-EI-MS: Autospec Premier (Waters).

GC-MS: were recorded on a GC system Agilent Technologies 7890-A series/Mass selective detector, Agilent Technologies 5975 C (column: HP-5MS (J\&W Scientific, Agilent); 30 m, 0.250 mm i.D., Film 0.25 $\mu \mathrm{m})$.

IR: spectra were measured on a JASCO FT/IR-4100 Spectrometer. Characteristic absorption bands are displayed in wavelengths $\tilde{\mathrm{v}}$ in $\mathrm{cm}^{-1}$ and were analyzed with the software Spectral Manager from JASCO.

Melting points were measured on a Thermovar from the company Reichert and are not corrected.

Chromatography Reaction progress was monitored by thin layer chromatography on aluminum backed silica gel plates (silica gel $60 \mathrm{~F} 254$ from E. Merck), visualizing with UV light $(\lambda=254 \mathrm{~nm}$ ). The plates were developed using vanillin dip solution $(170 \mathrm{~mL}$ methanol, $20.0 \mathrm{ml}$ conc. acetic acid, $10.0 \mathrm{~mL}$ conc. sulfuric acid with $1.0 \mathrm{~g}$ vanillin), $\mathrm{KMnO}_{4}$ dip solution ( $3.0 \mathrm{~g}$ potassium permanganate, $5.0 \mathrm{~mL} \mathrm{NaOH}$ solution $(5 \mathrm{w} / \mathrm{w}), 300 \mathrm{~mL}$ dest. water) or an anisaldehyde solution $(450 \mathrm{~mL}$ ethanol, $25.0 \mathrm{~mL}$ anisaldehyde, $25.0 \mathrm{~mL}$ conc. sulfuric acid, $8.0 \mathrm{~mL}$ acetic acid).

Flash chromatography was performed using silica gel M60 from Macherey \& Nagel (particle size: 40-63 $\mu \mathrm{m})$.

HPLC: were conducted on a modular Knauer HPLC system with a UV detector at $254 \mathrm{~nm}$ and differential refractometer on a 4 × $250 \mathrm{~mm}$ column packed with Nucleosil 50-5 from Machery-Nagel.

Flow Reactions: Flow reactions were performed in 1/16 inch PTFE tubing with an inner diameter of 1.0 $\mathrm{mm}$. The tubing was embedded in an aluminum block from ThalesNano and heated with an Ika stirring plate. A stainless-steel T-piece from Vici or static mixer (Upchurch Scientific) was used to mix the reagents. Fittings were either coned 10/32 stainless steel fittings from Upchurch scientific or flat bottom 1/4-28 gripper fittings from Dibafit. A kdScientific syringe pump (model no. KDS 200CE) was used to pump the reagents through the reactor.

Microwave Reactions: Reactions in a microwave were performed in a sealed tube using a Biotage Initiator Microwave with a maximum power of $400 \mathrm{~W}$. 
Reagents and Solvents Reactions with air or moisture-sensitive substances were, if not otherwise indicated, carried out under an argon atmosphere with the help of the Schlenk technique. All other reagents and solvents were used as purchased from commercial suppliers unless otherwise noted. Anhydrous solvents were purified with the solvent purification system MB-SPS-800 (Braun). Water free Acetonitrile was purchased from Acros Organics in AcroSeal@-bottles under Argon atmosphere with molecular sieves $(3 \AA)$ or HPLC-grade acetonitrile from Fischer Scientific was used. The solvents (ethyl acetate, pentane) used for column chromatography and work up were purified from commercially available technical grade solvents by distillation under reduced pressure with the help of rotatory evaporators (Heidolph or IKA) at $40^{\circ} \mathrm{C}$ water bath temperature.

Benzamides, ${ }^{1}$ benzyloxy acetic acid, ${ }^{2}$ benzyloxyacetyl chloride ${ }^{3}$ and 3-hydroxy-2-(trimethylsilyl)phenyl triflate $^{4}$ were prepared according to literature procedures.

Compound names are derived from Chemdraw and are not necessarily identical with the IUPAC nomenclature. 


\section{Synthesis of Compounds}

\section{$N-(2-M e t h o x y a c e t y l)-$ benzamide (4a)}

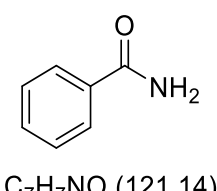

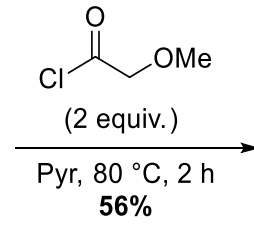

$56 \%$<smiles>COCC(=O)NC(=O)c1ccccc1</smiles>

$\mathrm{C}_{10} \mathrm{H}_{11} \mathrm{NO}_{3}(193.20)$

Benzamide (2.00 g, $16.5 \mathrm{mmol}, 1$ equiv.) was dissolved in anhydrous pyridine (26 mL) and 2methoxyacetyl chloride $(3.01 \mathrm{~mL}, 33.0 \mathrm{mmol}, 2$ equiv.) was added in one portion at room temperature. The yellow mixture was stirred in a sealed tube at $80^{\circ} \mathrm{C}$ for 2 hours. After cooling to room temperature, the mixture was concentrated under reduced pressure to $1 / 4$ of its volume and diluted with $\mathrm{NH}_{4} \mathrm{Cl}$ (sat. aq., $50 \mathrm{~mL}$ ) and EtOAc $(50 \mathrm{~mL})$. The organic layer was separated and the aqueous layer was extracted with EtOAc $(3 \times 50 \mathrm{~mL})$. The combined organic layers were washed with $\mathrm{NH}_{4} \mathrm{Cl}$ (sat. aq., $3 \times 150 \mathrm{~mL}$ ) and $\mathrm{NaCl}$ (sat. aq., $100 \mathrm{~mL}$ ), dried $\left(\mathrm{MgSO}_{4}\right)$, filtered and concentrated under reduced pressure. The crude product was purified by column chromatography (silica gel, $n$-pentane/EtOAc $=1: 1$ ) affording the title compound (la, $1.77 \mathrm{~g}, 9.17 \mathrm{mmol}, 56 \%$ ) as a colorless solid.

$\mathbf{R}_{\mathbf{f}}=0.41$ (n-pentane/EtOAc = 1:1); m.p.: $105{ }^{\circ} \mathrm{C} ;{ }^{1} \mathbf{H} \mathbf{N M R}\left(500 \mathrm{MHz}, \mathrm{CDCl}_{3}\right): \delta=9.44(\mathrm{~s}, 1 \mathrm{H}), 7.86(\mathrm{~d}$, $J=7.4 \mathrm{~Hz}, 2 \mathrm{H}$ ), 7.58 (t, $J=7.4 \mathrm{~Hz}, 1 \mathrm{H}), 7.48(\mathrm{t}, J=7.7 \mathrm{~Hz}, 2 \mathrm{H}), 4.38(\mathrm{~s}, 2 \mathrm{H}), 3.49$ (s, 3H) ppm; ${ }^{13} \mathrm{C}$ NMR $\left(126 \mathrm{MHz}, \mathrm{CDCl}_{3}\right): \delta=171.1,165.2,133.4,132.5,129.0,127.9,73.1,59.5$ ppm; IR (neat): $\tilde{v}=$ 3378, 3280, 3168, 3071, 2990, 2963, 2938, 2920, 2825, 2748, 2600, 1909, 1771, 1709, 1685, 1636, 1602, 1584, 1555, 1508, 1465, 1448, 1396, 1384, 1346, 1327, 1310, 1287, 1250, 1239, 1196, 1169 , 1120, 1090, 1069, 1031, 1012, 1002, 973, 933, 907, 842, 818, 805, 751, $701 \mathrm{~cm}^{-1} ;$ HRMS (ESI): m/z calculated for $\mathrm{C}_{10} \mathrm{H}_{11} \mathrm{NNaO}_{3}{ }^{+}\left([\mathrm{M}+\mathrm{Na}]^{+}\right): 216.0631$; found: 216.0641 .

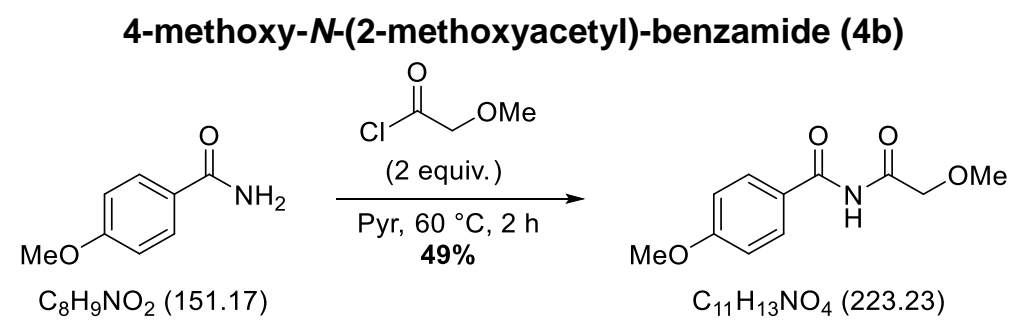

4-Methoxybenzamide ( $2.00 \mathrm{~g}, 13.2 \mathrm{mmol}, 1$ equiv.) was dissolved in anhydrous pyridine (20 mL) and 2methoxyacetyl chloride $(1.50 \mathrm{~mL}, 16.5 \mathrm{mmol}, 2$ equiv.) was added at room temperature. The orange mixture was stirred in a sealed tube at $60^{\circ} \mathrm{C}$ for 2 hours. After cooling to room temperature, the solvent was removed under reduced pressure. The residue was suspended in $\mathrm{NaHCO}_{3}$ (sat. aq., $250 \mathrm{~mL}$ ) and EtOAc $(250 \mathrm{~mL})$. The organic layer was separated and the aqueous layer was extracted with EtOAc $(3 \times 100 \mathrm{~mL})$. The combined organic layers were washed with $\mathrm{NaCl}$ (sat. aq., $2 \times 400 \mathrm{~mL}$ ), dried $\left(\mathrm{Na}_{2} \mathrm{SO}_{4}\right)$, filtered and concentrated under reduced pressure. The crude product was recrystallized from hot EtOAc affording the title compound (Ib, $1.45 \mathrm{~g}, 6.48 \mathrm{mmol}, 49 \%$ ) as a colorless solid.

$\mathbf{R}_{\mathbf{f}}=0.42$ (n-pentane/EtOAc = 1:2); m.p.: $156{ }^{\circ} \mathrm{C} ;{ }^{1} \mathbf{H}$ NMR $\left(400 \mathrm{MHz}\right.$, DMSO- $\left.d_{6}\right): \delta=11.00(\mathrm{~s}, 1 \mathrm{H}), 7.94$ (d, $J=8.8 \mathrm{~Hz}, 2 \mathrm{H}), 7.03(\mathrm{~d}, J=8.9 \mathrm{~Hz}, 2 \mathrm{H}), 4.44(\mathrm{~s}, 2 \mathrm{H}), 3.83(\mathrm{~s}, 3 \mathrm{H}), 3.34$ (s, 3H) ppm; ${ }^{13} \mathrm{C} \mathrm{NMR}(101$ 
MHz, DMSO- $\left.d_{6}\right): \delta=172.9,165.7,163.0,130.7,124.6,113.8,72.8,58.5,55.6$ ppm; IR (neat): $\tilde{v}=$ 3390, 3291, 3169, 3094, 3080, 3014, 2970, 2942, 2843, 1769, 1710, 1685, 1645, 1618, 1607, 1574, 1517, 1458, 1422, 1394, 1311, 1254, 1182, 1146, 1124, 1025, 849, 809, $764 \mathrm{~cm}^{-1} ;$ HRMS (ESI): m/z calculated for $\mathrm{C}_{11} \mathrm{H}_{13} \mathrm{NNaO}_{4}+\left([\mathrm{M}+\mathrm{Na}]^{+}\right)$: 246.0737; found 246.0738 .

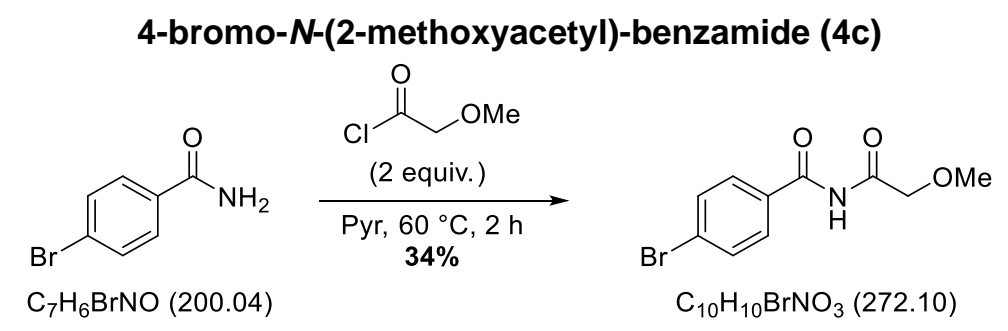

4-Bromobenzamide (150 mg, $0.992 \mathrm{mmol}, 1$ equiv.) was dissolved in anhydrous pyridine (1.6 mL) and 2-methoxyacetyl chloride $(0.181 \mathrm{~mL}, 1.99 \mathrm{mmol}, 2$ equiv.) was added at room temperature. The orange mixture was stirred in a sealed tube at $60^{\circ} \mathrm{C}$ for 2 hours. After cooling to room temperature, the solvent was removed under reduced pressure. The residue was suspended in $\mathrm{NaHCO}_{3}$ (sat. aq., $20 \mathrm{~mL}$ ) and $\mathrm{EtOAc}(20 \mathrm{~mL})$. The organic layer was separated and the aqueous layer was extracted with EtOAc $(3 \times 20 \mathrm{~mL})$. The combined organic layers were washed with $\mathrm{NaCl}$ (sat. aq., $2 \times 50 \mathrm{~mL})$, dried $\left(\mathrm{Na}_{2} \mathrm{SO}_{4}\right)$, filtered and concentrated under reduced pressure. The crude product was purified by column chromatography (silica gel, $n$-pentane/EtOAc 2:1) to afford the title compound (Ic, $74.0 \mathrm{mg}, 0.332 \mathrm{mmol}$, $34 \%)$ as a colorless solid.

$\mathbf{R}_{\mathbf{f}}=0.30$ (n-pentane/EtOAc = 1:2); m.p.: $141^{\circ} \mathrm{C} ;{ }^{1} \mathbf{H}$ NMR $\left(500 \mathrm{MHz}, \mathrm{CDCl}_{3}\right) \delta=9.37(\mathrm{~s}, 1 \mathrm{H}), 7.73(\mathrm{~d}$, $J=8.1 \mathrm{~Hz}, 2 \mathrm{H}), 7.64(\mathrm{~d}, J=8.2 \mathrm{~Hz}, 2 \mathrm{H}), 4.35(\mathrm{~s}, 2 \mathrm{H}), 3.51$ (s, 3H) ppm; ${ }^{13} \mathrm{C} \mathrm{NMR}\left(126 \mathrm{MHz}, \mathrm{CDCl}_{3}\right) \delta=$ 170.7, 164.5, 132.4, 131.5, 129.5, 128.6, 73.0, 59.6 ppm; IR (neat): $\tilde{v}=3240,3196,3160,2970,2952$, 2926, 2823, 1731, 16696, 1591, 1524, 1502, 1479, 1400, 1386, 1257, 1232, 1202, 1136, 1110, 1069, 1012, 939, 907, 840, 818, 772, 763, 744, 717, 684, $661 \mathrm{~cm}^{-1}$; HRMS (ESI): m/z calculated for $\mathrm{C}_{10} \mathrm{H}_{10} \mathrm{BrNO}_{3} \mathrm{Na}^{+}\left([\mathrm{M}+\mathrm{Na}]^{+}\right): 293.9736$; found: 293.9745.

\section{$N$-(2-(benzyloxy)acetyl)benzamide (4d)}
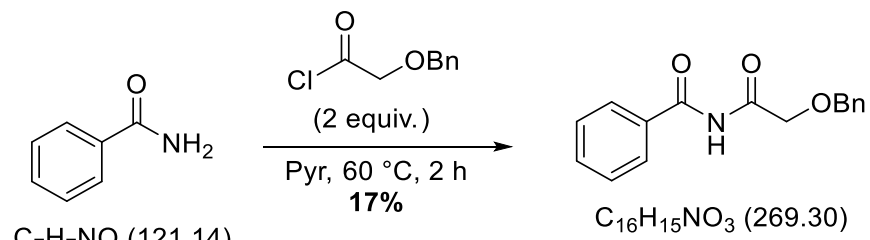

Benzamide (800 mg, $6.60 \mathrm{mmol}, 1$ equiv.) was dissolved in anhydrous pyridine $(11 \mathrm{~mL})$. Benzyloxyacetyl chloride $(2.44 \mathrm{~g}, 13.2 \mathrm{mmol}, 2$ equiv.) was added and the mixture was stirred in a sealed tube at $60^{\circ} \mathrm{C}$ for $2 \mathrm{~h}$. After cooling to room temperature, the solvent was removed under reduced pressure. The residue was dissolved in $\mathrm{EtOAc}(10 \mathrm{~mL})$ and $\mathrm{NaHCO}_{3}$ (sat. aq., $10 \mathrm{~mL}$ ), the layers were separated and the aqueous layer was extracted with EtOAc $(3 \times 10 \mathrm{~mL})$. The combined organic layers were washed with $\mathrm{NaCl}$ (sat. aq., $2 \times 10 \mathrm{~mL}$ ) and dried $\left(\mathrm{MgSO}_{4}\right)$. The solvents were removed under reduced pressure. The crude product was recrystallized from hot EtOAc affording the title compound (Id, $300 \mathrm{mg}, 1.10 \mathrm{mmol}, 17 \%$ ) as a colorless solid. 
m.p.: $113^{\circ} \mathrm{C} ;{ }^{1} \mathrm{H}$ NMR $\left(700 \mathrm{MHz}, \mathrm{CDCl}_{3}\right) \delta=9.32(\mathrm{~s}, 1 \mathrm{H}), 7.82-7.80(\mathrm{~m}, 2 \mathrm{H}), 7.60(\mathrm{td}, J=7.3,1.3 \mathrm{~Hz}$, $1 \mathrm{H}), 7.50-7.47(\mathrm{~m}, 2 \mathrm{H}), 7.39(\mathrm{~s}, 2 \mathrm{H}), 7.38(\mathrm{~d}, J=1.6 \mathrm{~Hz}, 2 \mathrm{H}), 7.36-7.33(\mathrm{~m}, 1 \mathrm{H}), 4.70(\mathrm{~s}, 2 \mathrm{H}), 4.43$ (s, $2 \mathrm{H})$ ppm; ${ }^{13} \mathrm{C}$ NMR $\left(176 \mathrm{MHz}, \mathrm{CDCl}_{3}\right) \delta=170.5,165.0,136.8,133.5,132.6,129.2,128.8,128.5$, 128.2, 127.8, 73.9, 70.5 ppm; IR (neat): $\tilde{v}=3284,2951,2922,2868,1712,1688,1599,1582,1500$, 1469, 1406, 1390, 1373, 1324, 1304, 1244, 1216, 1115, 1102, 1072, 1028, 1001, 975, 949, 933, 908, 872, 862, 841, 822, 800, 785, 757, 702, $657 \mathrm{~cm}^{-1}$; HRMS (ESI): $\mathrm{m} / z$ calculated for $\mathrm{C}_{16} \mathrm{H}_{15} \mathrm{NNaO}_{3}{ }^{+}$ $\left([\mathrm{M}+\mathrm{Na}]^{+}\right): 292.0944 ;$ found: 292.0951 .

\section{3-(benzyloxy)-2-(trimethylsilyl)phenyl trifluoromethanesulfonate (3b)}

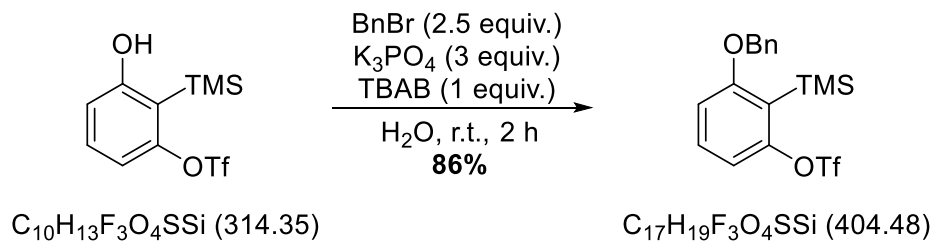

3-Hydroxy-2-trimethylsilyl-phenyl trifluoromethanesulfonate $(500 \mathrm{mg}, 1.59 \mathrm{mmol}, 1$ equiv.) was suspended in water $(15 \mathrm{~mL})$ and $\mathrm{BnBr}(0.472 \mathrm{~mL}, 3.98 \mathrm{mmol}, 2.5$ equiv.), TBAB (513 mg, $1.59 \mathrm{mmol}$, 1 equiv.) and $\mathrm{K}_{3} \mathrm{PO}_{4}(1.01 \mathrm{~g}, 4.77 \mathrm{mmol}, 3$ equiv.) were added at room temperature. The suspension was stirred vigorously at room temperature for two hours, diluted with water and extracted with EtOAc $(3 \times 50 \mathrm{~mL})$. The combined organic layers were dried $\left(\mathrm{Na}_{2} \mathrm{SO}_{4}\right)$, filtered and concentrated under reduced pressure. The crude product was purified by column chromatography (silica gel, $n$-pentane/EtOAc $=$ 20:1) to afford the title compound (IVb, $555 \mathrm{mg}, 1.37 \mathrm{mmol}, 86 \%$ ) as a colorless oil.

$\mathbf{R}_{\mathbf{f}}=0.83\left(n\right.$-pentane/EtOAc = 10:1); ${ }^{1} \mathbf{H}$ NMR $\left(500 \mathrm{MHz}, \mathrm{CDCl}_{3}\right) \delta=7.41(\mathrm{~s}, 4 \mathrm{H}), 7.39-7.32(\mathrm{~m}, 2 \mathrm{H})$, $6.97(\mathrm{~d}, J=8.4 \mathrm{~Hz}, 1 \mathrm{H}), 6.89(\mathrm{~d}, J=8.4 \mathrm{~Hz}, 1 \mathrm{H}), 5.09(\mathrm{~s}, 2 \mathrm{H}), 0.34(\mathrm{~s}, 9 \mathrm{H}) \mathrm{ppm} ;{ }^{13} \mathrm{C} \mathrm{NMR}(126 \mathrm{MHz}$, $\left.\mathrm{CDCl}_{3}\right) \delta=164.8,154.9,136.2,131.7,128.8,128.4,127.9,121.2,120.1,117.5,113.1,110.6,71.1,1.1$ ppm; ${ }^{19} \mathrm{~F}$ NMR (376 MHz, $\left.\mathrm{CDCl}_{3}\right) \delta=-73.98$ ppm; IR (neat): $\tilde{v}=3067,3036,2954,2901,2876,1594$, 1565, 1434, 1417, 1247, 1207, 1160, 1137, 1116, 1024, 934, 842, 826, 785, $735 \mathrm{~cm}^{-1}$; HRMS (ESI): m/z calculated for $\mathrm{C}_{17} \mathrm{H}_{19} \mathrm{~F}_{3} \mathrm{SSiNa}\left([\mathrm{M}+\mathrm{Na}]^{+}\right): 427.0617$, found 427.0667 .

\section{3-(allyloxy)-2-(trimethylsilyl)phenyl trifluoromethanesulfonate (3c)}

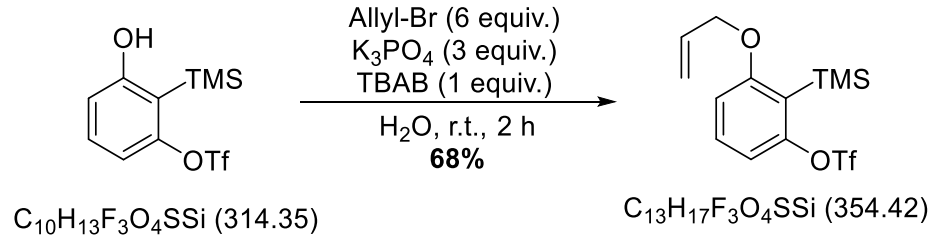

3-Hydroxy-2-trimethylsilyl-phenyl trifluoromethanesulfonate $(1.47 \mathrm{~g}, 4.66 \mathrm{mmol}, 1$ equiv.) was suspended in water (150 mL). Allyl bromide ( $2.42 \mathrm{~mL}, 28.0 \mathrm{mmol}, 6.0$ equiv.), TBAB (1.50 g, $4.66 \mathrm{mmol}$, 1.0 equiv.) and $\mathrm{K}_{3} \mathrm{PO}_{4}(2.97 \mathrm{~g}, 14.0 \mathrm{mmol}, 3$ equiv.) were added at room temperature. The suspension was stirred vigorously at room temperature for one hour, diluted with water $(50 \mathrm{~mL})$ and extracted with EtOAc $(3 \times 50 \mathrm{~mL})$. The combined organic layers were dried $\left(\mathrm{Na}_{2} \mathrm{SO}_{4}\right)$, filtered and concentrated under reduced pressure. The crude product was purified by column chromatography (silica gel, $n$-pentane) to afford the title compound (IVc, $1.12 \mathrm{~g}, 3.16 \mathrm{mmol}, 68 \%$ ) as a colorless oil. 
$\mathbf{R}_{\mathbf{f}}=0.65$ (n-pentane); ${ }^{1} \mathrm{H}$ NMR $\left(500 \mathrm{MHz}, \mathrm{CDCl}_{3}\right) \delta=7.34(\mathrm{t}, J=8.4 \mathrm{~Hz}, 1 \mathrm{H}), 6.94(\mathrm{~d}, J=8.4 \mathrm{~Hz}, 1 \mathrm{H})$, $6.81(\mathrm{~d}, J=8.3 \mathrm{~Hz}, 1 \mathrm{H}), 6.09-5.98(\mathrm{~m}, 1 \mathrm{H}), 5.45-5.35(\mathrm{~m}, 1 \mathrm{H}), 5.34-5.30(\mathrm{~m}, 1 \mathrm{H}), 4.56(\mathrm{~d}, J=5.4$ $\mathrm{Hz}, 2 \mathrm{H}), 0.38$ (s, 9H) ppm; ${ }^{13} \mathrm{C}$ NMR $\left(126 \mathrm{MHz}, \mathrm{CDCl}_{3}\right) \delta=164.6,154.8,132.7,131.7,121.2,118.5$, 117.5, 113.0, 110.6, 69.7, $1.1 \mathrm{ppm} ;{ }^{19} \mathrm{~F}$ NMR $\left(376 \mathrm{MHz}, \mathrm{CDCl}_{3}\right) \delta=-72.77 \mathrm{ppm}$; IR (neat): $\tilde{v}=3089$, 2988, 2955, 2901, 2865, 1595, 1565, 1436, 1420, 1362, 1247, 1206, 1160, 1140, 1117, 1057, 1034, 996, 939, 924, 894, 837, 787, 769, 738, 712, 693, $668 \mathrm{~cm}^{-1}$; HRMS (ESI): m/z calculated for $\mathrm{C}_{13} \mathrm{H}_{17} \mathrm{~F}_{3} \mathrm{O}_{4} \mathrm{SSiNa}^{+}\left([\mathrm{M}+\mathrm{Na}]^{+}\right): 377.0461$, found: 377.0461 .

Table S1. Analysis of main parameters for the synthesis of benzophenone $\mathbf{5 a}$ and acetophenone $\mathbf{7}$

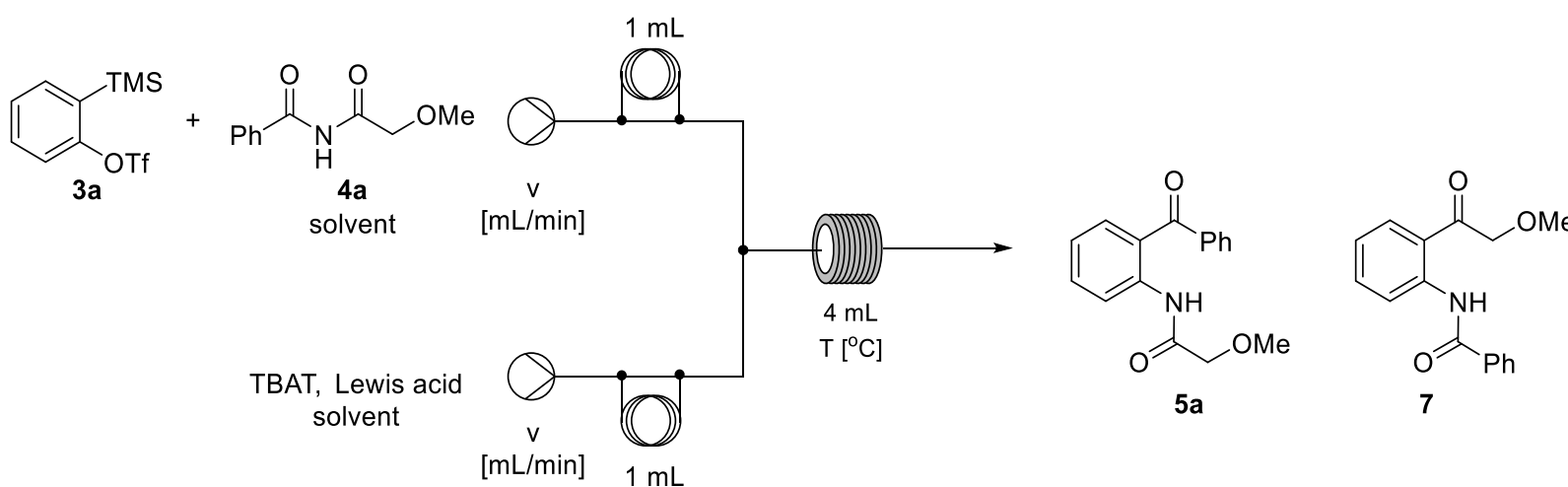

\begin{tabular}{|c|c|c|c|c|c|c|c|c|c|c|}
\hline Entry & $\begin{array}{c}v \\
{[\mathrm{ml} / \mathrm{min}]}\end{array}$ & $\begin{array}{l}\text { Residence } \\
\text { time } \\
\text { [min] }\end{array}$ & $\begin{array}{l}\text { Lewis } \\
\text { acid }\end{array}$ & $\begin{array}{c}C_{\text {Imid }} \\
{[\mathrm{mol} / \mathrm{L}]}\end{array}$ & $\begin{array}{c}C_{\text {Arin }} \\
{[\mathrm{mol} / \mathrm{L}]}\end{array}$ & $\begin{array}{c}\mathrm{C}_{\text {ТвAT }} \\
{[\mathrm{mol} / \mathrm{L}]}\end{array}$ & $\mathrm{T}\left[{ }^{\circ} \mathrm{C}\right]$ & Ila : IIIa & Solvent/cond. & $\begin{array}{l}5 \mathrm{a}^{\mathrm{a}} \\
{[\%]}\end{array}$ \\
\hline 1 & 0.5 & 4 & none & 0.05 & 0.06 & 0.10 & 55 & - & no workup & 32 \\
\hline 2 & 0.5 & 4 & none & 0.05 & 0.06 & 0.10 & 55 & - & $\begin{array}{l}\text { aqueous } \\
\text { workup }\end{array}$ & 42 \\
\hline 3 & 0.5 & 4 & none & 0.05 & 0.06 & 0.10 & 55 & - & $\begin{array}{c}\text { wet MeCN, no } \\
\text { workup, static } \\
\text { mixer }\end{array}$ & 31 \\
\hline 4 & 0.5 & 4 & none & 0.05 & 0.06 & 0.10 & 55 & - & $\begin{array}{c}\text { static mixer, dry } \\
\text { MeCN }\end{array}$ & 36 \\
\hline 5 & 0.2 & 10 & none & 0.05 & 0.06 & 0.10 & 55 & - & $\begin{array}{c}\text { wet MeCN, no } \\
\text { workup, static } \\
\text { mixer }\end{array}$ & 32 \\
\hline 6 & 0.2 & 10 & none & 0.05 & 0.06 & 0.10 & 65 & - & $\begin{array}{c}\text { werkup, static } \\
\text { mixer }\end{array}$ & 32 \\
\hline 7 & 0.2 & 10 & none & 0.05 & 0.08 & 0.10 & 55 & - & $\begin{array}{c}\text { wet MeCN, no } \\
\text { workup, static } \\
\text { mixer }\end{array}$ & 26 \\
\hline 8 & 0.2 & 10 & none & 0.09 & 0.05 & 0.10 & 55 & - & $\begin{array}{c}\text { wet MeCN, no } \\
\text { workup, static } \\
\text { mixer }\end{array}$ & 24 \\
\hline 9 & 0.5 & 4 & none & 0.05 & 0.08 & 0.10 & 80 & - & $\begin{array}{c}\text { wet MeCN, no } \\
\text { workup, static } \\
\text { mixer }\end{array}$ & 42 \\
\hline 10 & 0.5 & 4 & none & 0.025 & 0.04 & 0.05 & 55 & $2.7: 1$ & $\begin{array}{c}\mathrm{PhMe} / \mathrm{MeCN} \\
(1: 1)\end{array}$ & 31 \\
\hline 11 & 0.5 & 4 & none & 0.025 & 0.04 & 0.05 & 80 & $4.2: 1$ & $\begin{array}{c}\text { PhMe/MeCN } \\
(1: 1) ; 40 \text { psi BPR }\end{array}$ & 42 \\
\hline
\end{tabular}




\begin{tabular}{|c|c|c|c|c|c|c|c|c|c|c|}
\hline 12 & 0.2 & 10 & none & 0.025 & 0.04 & 0.05 & 65 & $3.1: 1$ & $\begin{array}{c}\mathrm{PhMe} / \mathrm{MeCN} \\
(1: 1), \text { static } \\
\text { mixer }\end{array}$ & 32 \\
\hline 13 & 0.5 & 4 & none & 0.05 & 0.08 & 0.1 & 55 & $2.5: 1$ & THF & 20 \\
\hline 14 & 0.5 & 4 & none & 0.04 & 0.08 & 0.1 & 80 & $4.1: 1$ & $\begin{array}{c}\text { wet MeCN; } 40 \\
\text { psi BPR }\end{array}$ & 36 \\
\hline 15 & 0.5 & 4 & none & 0.04 & 0.08 & 0.1 & 90 & $4.2: 1$ & $\begin{array}{c}\text { wet MeCN; } 40 \\
\text { psi BPR }\end{array}$ & 35 \\
\hline 16 & 0.5 & 4 & none & 0.04 & 0.08 & 0.1 & 100 & $4.0: 1$ & $\begin{array}{c}\text { wet MeCN; } 40 \\
\text { psi BPR }\end{array}$ & 40 \\
\hline 17 & 0.5 & 4 & $\mathrm{Sc}(\mathrm{OTf})_{3}$ & 0.1 & 0.15 & 0.18 & 65 & - & dry MeCN & $<5^{b}$ \\
\hline 18 & 0.5 & 4 & $\mathrm{MgCl}_{2}$ & 0.1 & 0.15 & 0.18 & 65 & - & dry MeCN & $<5^{b}$ \\
\hline 19 & 0.5 & 4 & $\mathrm{BF}_{3} \cdot \mathrm{OEt}_{2}$ & 0.1 & 0.15 & 0.18 & 65 & - & dry MeCN & $<5^{b}$ \\
\hline 20 & 0.5 & 4 & $\mathrm{AlCl}_{3}$ & 0.1 & 0.15 & 0.18 & 65 & - & dry MeCN & $<5^{b}$ \\
\hline 21 & 0.5 & 4 & $\mathrm{Ti}(\mathrm{OiPr})_{4}$ & 0.1 & 0.15 & 0.18 & 65 & - & dry MeCN & $<5^{b}$ \\
\hline 22 & 0.5 & 4 & $\mathrm{Ti}(\mathrm{OiPr})_{4}$ & 0.1 & 0.15 & 0.18 & 65 & - & dry MeCN & $<5^{b}$ \\
\hline 23 & 0.5 & 4 & none & 0.1 & 0.15 & 0.18 & 65 & $2.9: 1^{c}$ & wet $\mathrm{MeCN}$ & $52^{c}$ \\
\hline 24 & 0.2 & 10 & none & 0.05 & 0.06 & 0.075 & 65 & $3.3: 1^{b}$ & dry MeCn & $42^{c}$ \\
\hline 25 & 0.2 & 10 & none & 0.05 & 0.06 & 0.075 & 80 & $3.8: 1^{b}$ & dry MeCN & $39^{c}$ \\
\hline
\end{tabular}

aThe ratio of $5 \mathbf{a}: 7$ as well as the yield of lla were determined via GC-MS acetanilid as standard. An aliquot of the reactor output ( $100 \mu \mathrm{L}$ or $500 \mu \mathrm{L}$ ) was taken, diluted to yield a volume of $900 \mu \mathrm{L}$ and treated with a solution of acetanilide $(100 \mu \mathrm{L}, 0.05 \mathrm{M})$. ${ }^{\mathrm{b}}$ Determined by ${ }^{1} \mathrm{H}$ NMR integration. cisolated yield.

\section{General Procedure for the Aryne Insertion in Flow (GP1)}

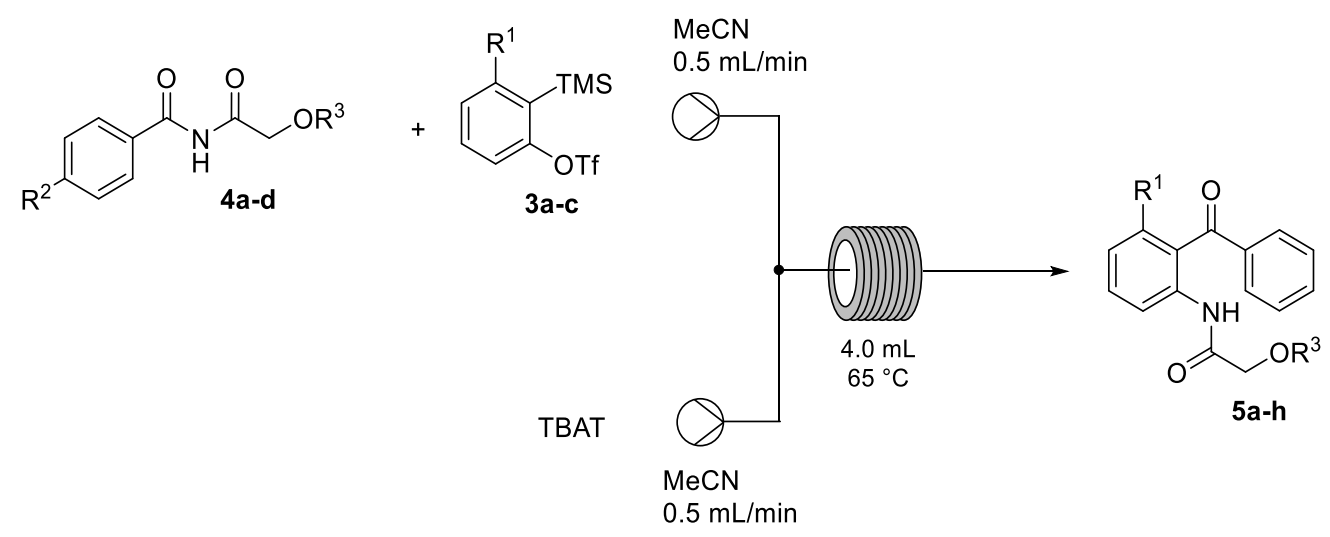

Prior use, the flow reactor was washed with three reactor volumes of acetonitrile using a syringe pumps. A stock solution of aryne precursor (3a-c, $0.150 \mathrm{M}$ in MeCN, 1.5 equiv.) and imide (4a-d, $0.100 \mathrm{M}$ in 
MeCN, 1.0 equiv.) was loaded on a sample loop with a volume of $1 \mathrm{~mL}$. Then, a stock solution of TBAT $(0.180 \mathrm{M}$ in acetonitrile, 1.8 equiv.) was loaded on a sample loop with a volume of $1 \mathrm{~mL}$. Both solutions were pumped simultaneously at a rate of $0.5 \mathrm{~mL} / \mathrm{min}$, driven by $\mathrm{MeCN}$. The stock solutions were combined at a T-piece to react in a $4.0 \mathrm{~mL}$ PTFE coil, preheated to $65^{\circ} \mathrm{C}$ using an aluminum block. The reaction mixture was collected, concentrated under reduced pressure and purified by column chromatography to afford the ortho-aminobenzophenones $5 \mathrm{a}-\mathrm{h}$.

\section{$N$-(2-benzoylphenyl)-2-methoxyacetamide (5a)}
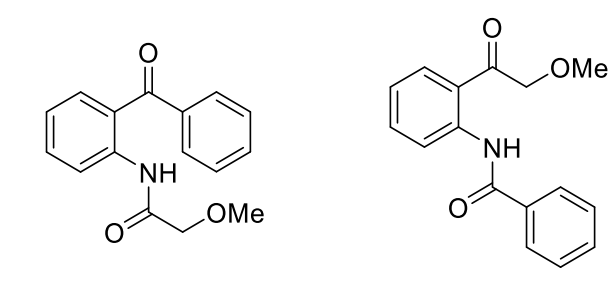

5a $\mathrm{C}_{16} \mathrm{H}_{15} \mathrm{NO}_{3}(269.30)$

$7 \mathrm{C}_{16} \mathrm{H}_{15} \mathrm{NO}_{3}(269.30)$

Compound 5a was prepared according to GP1 starting from $3 \mathbf{a}$ (127 mg, $0.427 \mathrm{mmol}, 1.5$ equiv.) and 4a $(55.0 \mathrm{mg}, 0.285 \mathrm{mmol}, 1$ equiv.). Column chromatography (silica gel, $n$-pentane/EtOAc $=4: 1$ ) afforded the title compound ( $5 \mathrm{a}, 40.0 \mathrm{mg}, 0.148 \mathrm{mmol}, 52 \%$ ) as a colorless oil that solidified in the fridge and compound 7 (14.5 mg, $0.054 \mathrm{mmol}, 19 \%$ ) as a colorless solid.

5a:

$\mathbf{R}_{\mathbf{f}}=0.40\left(n\right.$-pentane/EtOAc = 3:1); ${ }^{1} \mathbf{H}$ NMR $\left(700 \mathrm{MHz}, \mathrm{CDCl}_{3}\right): \delta=11.39(\mathrm{~s}, 1 \mathrm{H}), 8.69(\mathrm{~d}, J=8.3 \mathrm{~Hz}$, $1 \mathrm{H}), 7.73-7.70(\mathrm{~m}, 2 \mathrm{H}), 7.60-7.54(\mathrm{~m}, 3 \mathrm{H}), 7.47$ (t, $J=7.8 \mathrm{~Hz}, 2 \mathrm{H}), 7.11$ (td, $J=7.6,1.2 \mathrm{~Hz}, 1 \mathrm{H}$ ), 4.04 (s, 2H), 3.55 (s, 3H) ppm; ${ }^{13} \mathrm{C}$ NMR (176 MHz, $\left.\mathrm{CDCl}_{3}\right): \delta$ 199.1, 169.2, 139.4, 138.7, 134.1, 133.5, 132.5, 130.0, 128.4, 124.3, 122.6, 121.7, 72.7, 59.8 ppm; IR (neat): $\tilde{v}=3286,3060,3033,2996,2934$, 2828, 2756, 2249, 1832, 1692, 1639, 1598, 1577, 1515, 1446, 1432, 1362, 1317, 1293, 1263, 1196, 1180, 1158, 1114, 1076, 1048, 1028, 987, 959, 935, 917, 880, 852, 805, 752, $728 \mathrm{~cm}^{-1}$; HRMS (ESI): $\mathrm{m} / \mathrm{z}$ calculated for $\mathrm{C}_{16} \mathrm{H}_{15} \mathrm{NNaO}_{3}{ }^{+}\left([\mathrm{M}+\mathrm{Na}]^{+}\right)$: 292.0944; found: 292.0959 .

\section{7:}

$\mathbf{R}_{\mathbf{f}}=0.35$ (n-pentane/EtOAc = 3:1); m.p.: $138-139{ }^{\circ} \mathrm{C} ;{ }^{1} \mathbf{H} \mathbf{N M R}\left(500 \mathrm{MHz}, \mathrm{CDCl}_{3}\right): \delta=12.57(\mathrm{~s}, 1 \mathrm{H})$, $9.02(\mathrm{dd}, \mathrm{J}=8.6,1.1 \mathrm{~Hz}, 1 \mathrm{H}), 8.12-8.08(\mathrm{~m}, 2 \mathrm{H}), 7.82(\mathrm{dd}, \mathrm{J}=8.0,1.4 \mathrm{~Hz}, 1 \mathrm{H}), 7.64$ (ddd, J = 8.7, $7.3,1.5 \mathrm{~Hz}, 1 \mathrm{H}), 7.58-7.54(\mathrm{~m}, 1 \mathrm{H}), 7.53-7.49(\mathrm{~m}, 2 \mathrm{H}), 7.14(\mathrm{t}, \mathrm{J}=8.2 \mathrm{~Hz}, 1 \mathrm{H}), 4.81(\mathrm{~s}, 2 \mathrm{H}), 3.55(\mathrm{~s}$, 3H) ppm; ${ }^{13} \mathrm{C}$ NMR $\left(126 \mathrm{MHz}, \mathrm{CDCl}_{3}\right): \delta=200.2,166.2,141.8,135.9,134.6,132.2,129.6,129.0,127.7$, 122.6, 121.3, 119.7, 75.5, 59.7 ppm; IR (neat): $\tilde{v}=3268,3236,3130,3062,2995,2942,2829,1672$, $1653,1610,1585,1559,1537,1507,1496,1448,1368,1322,1311,1258,1216,1196,1139,1120$, 1029, 978, 925, 699, $661 \mathrm{~cm}^{-1}$; HRMS (ESI): $\mathrm{m} / \mathrm{z}$ calculated for $\mathrm{C}_{16} \mathrm{H}_{15} \mathrm{NNaO}_{3}{ }^{+}\left([\mathrm{M}+\mathrm{Na}]^{+}\right): 292.0944$; found: 292.0944 . 


\section{2-methoxy-N-(2-(4-methoxybenzoyl)phenyl)acetamide (5b)}

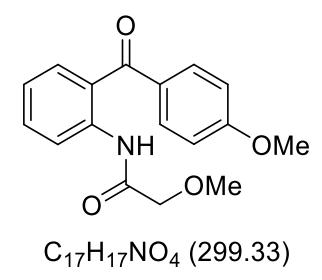

Compound 5b was prepared according to GP1 starting from 3a $(90.4 \mathrm{mg}, 0.303 \mathrm{mmol}, 1.5$ equiv., $0.015 \mathrm{M})$ and and $\mathbf{4 b}(45.0 \mathrm{mg}, 0.202 \mathrm{mmol}, 1$ equiv., $0.010 \mathrm{M})$. Column chromatography (silica gel, $n$ pentane/EtOAc $=3: 1)$ afforded the title compound $(5 \mathrm{~b}, 32.8 \mathrm{mg}, 0.122 \mathrm{mmol}, 60 \%)$ as a slightly yellow oil.

$\mathbf{R}_{\mathbf{f}}=0.49(n$-pentane/EtOAc $=2: 1) ;{ }^{1} \mathbf{H}$ NMR $\left(500 \mathrm{MHz}, \mathrm{CDCl}_{3}\right): \delta=11.09(\mathrm{~s}, 1 \mathrm{H}), 8.62(\mathrm{dt}, J=8.1$, $1.0 \mathrm{~Hz}, 1 \mathrm{H}), 7.78-7.72(\mathrm{~m}, 2 \mathrm{H}), 7.58-7.52(\mathrm{~m}, 2 \mathrm{H}), 7.16-7.09(\mathrm{~m}, 1 \mathrm{H}), 6.99-6.93(\mathrm{~m}, 2 \mathrm{H}), 4.03(\mathrm{~s}$, 2H), 3.89 (s, 3H), 3.53 (s, 3H) ppm; ${ }^{13} \mathrm{C} \mathrm{NMR}\left(126 \mathrm{MHz}, \mathrm{CDCl}_{3}\right): \delta=197.3,169.1,163.5,138.8,133.4$, 132.8, 132.7, 131.1, 125.3, 122.7, 121.9, 113.7, 72.7, 59.8, 55.7 ppm; IR (neat): $\tilde{v}=3310,3006,2931$, 2840, 1687, 1633, 1597, 1578, 1510, 1447, 1419, 1315, 1306, 1293, 1253, 1196, 1172, 1154, 1110, 1026, 986, 925, 844, 788, 760, 739, $696 \mathrm{~cm}^{-1}$; HRMS (ESI): $\mathrm{m} / \mathrm{z}$ calculated for $\mathrm{C}_{16} \mathrm{H}_{15} \mathrm{NNaO}_{3}{ }^{+}\left([\mathrm{M}+\mathrm{Na}]^{+}\right)$: 322.1050; found: 322.1062 .

\section{$N$-(2-(4-bromobenzoyl)phenyl)-2-methoxyacetamide (5c)}

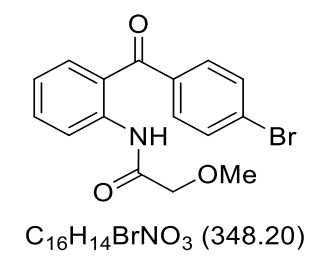

Compound 5c was prepared according to GP1 starting from $3 \mathbf{3 a}(44.8 \mathrm{mg}, 0.150 \mathrm{mmol}, 1.5$ equiv.) 4c (27.2 mg, $0.100 \mathrm{mmol}, 1$ equiv.). Column chromatography (silica gel, $n$-pentane/EtOAc 3:1) afforded the title compound (5c, $8.10 \mathrm{mg}, 0.023 \mathrm{mmol}, 23 \%$ ) as a slightly yellow oil.

$\mathbf{R}_{\mathbf{f}}=0.33\left(n\right.$-pentane/EtOAc = 3:1); ${ }^{1} \mathrm{H}$ NMR $\left(500 \mathrm{MHz}, \mathrm{CDCl}_{3}\right): \delta=11.31(\mathrm{~s}, 1 \mathrm{H}), 8.68(\mathrm{dd}, J=8.4,1.0$ $\mathrm{Hz}, 1 \mathrm{H}), 7.67-7.54(\mathrm{~m}, 5 \mathrm{H}), 7.52(\mathrm{dd}, J=7.9,1.6 \mathrm{~Hz}, 1 \mathrm{H}), 7.15-7.11(\mathrm{~m}, 1 \mathrm{H}), 4.05(\mathrm{~s}, 2 \mathrm{H}), 3.56(\mathrm{~s}$, 3H) ppm; ${ }^{13} \mathrm{C}$ NMR $\left(126 \mathrm{MHz}, \mathrm{CDCl}_{3}\right): \delta=197.9,169.2,139.5,137.5,134.4,133.2,131.8,131.6,127.7$, 124.0, 122.8, 121.9, 72.7, 59.9 ppm; IR (neat): $\tilde{v}=3298,3081,3033,2995,2932,2827,1692,1641$, $1601,1577,1515,1483,1446,1432,1394,1361,1314,1293,1262,1196,1178,1167,1157,1114$, 1068, 1051, 1010, 987, 959, 921, 880, 841, 783, 758, 725, 675, $654 \mathrm{~cm}^{-1} ;$ HRMS (ESI): m/z calculated for $\mathrm{C}_{16} \mathrm{H}_{14} \mathrm{BrNNaO}_{3}+\left([\mathrm{M}+\mathrm{Na}]^{+}\right)$: 370.0049 ; found: 370.0063 . 


\section{$N$-(2-benzoyl-3-(benzyloxy)phenyl)-2-methoxyacetamide (5d)}

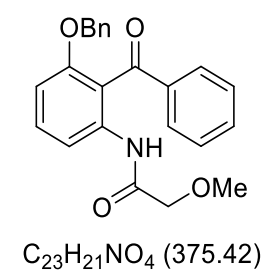

Compound $\mathbf{5 d}$ was prepared according to GP1 starting from $\mathbf{3 b}(62.0 \mathrm{mg}, 0.153 \mathrm{mmol}, 1.5$ equiv.) and 4a (44.6 mg, $0.230 \mathrm{mmol}, 1$ equiv.). Column chromatography (silica gel, $n$-pentane/EtOAc = 3:1) afforded the title compound ( $\mathbf{5 d}, 30.6 \mathrm{mg}, 0.082 \mathrm{mmol}, 35 \%$ ) as a slightly yellow oil.

$\mathbf{R}_{\mathbf{f}}=0.25(n$-pentane/EtOAc $=4: 1) ;{ }^{1} \mathbf{H}$ NMR $\left(700 \mathrm{MHz}, \mathrm{CDCl}_{3}\right): \delta=9.51(\mathrm{~s}, 1 \mathrm{H}), 8.03(\mathrm{dd}, J=8.4,0.8$ $\mathrm{Hz}, 1 \mathrm{H}), 7.80-7.77(\mathrm{~m}, 2 \mathrm{H}), 7.55(\mathrm{tt}, J=8.7,1.3 \mathrm{~Hz}, 1 \mathrm{H}), 7.47-7.41(\mathrm{~m}, 3 \mathrm{H}), 7.21-7.17(\mathrm{~m}, 1 \mathrm{H})$, $7.16-7.13(\mathrm{~m}, 2 \mathrm{H}), 6.81(\mathrm{dd}, J=8.4,0.8 \mathrm{~Hz}, 1 \mathrm{H}), 6.77(\mathrm{~d}, J=7.5 \mathrm{~Hz}, 2 \mathrm{H}), 4.90(\mathrm{~s}, 2 \mathrm{H}), 3.93(\mathrm{~s}, 2 \mathrm{H})$, 3.41 (s, 3H) ppm; ${ }^{13} \mathrm{C}$ NMR $\left(176 \mathrm{MHz}, \mathrm{CDCl}_{3}\right): \delta=197.1,168.6,157.4,139.4,137.1,136.1,133.1$, $132.5,129.4,128.5,128.4,127.8,126.8,118.8,115.2,108.5,72.4,70.4,59.6$ ppm; IR (neat): $\tilde{v}=3359$, $3061,3031,3003,2929,2829,1802,1693,1646,1597,1581,1523,1497,1466,1460,1450,1428$, $1381,1313,1275,1256,1196,1178,1146,1112,1087,1071,1028,986,925,875,865,846,807,782$, $739 \mathrm{~cm}^{-1} ;$ HRMS (ESI): $\mathrm{m} / \mathrm{z}$ calculated for $\mathrm{C}_{23} \mathrm{H}_{21} \mathrm{NNaO}^{+}\left([\mathrm{M}+\mathrm{Na}]^{+}\right): 398.1363$; found: 398.1367 .

\section{N-(3-(benzyloxy)-2-(4-methoxybenzoyl)phenyl)-2-methoxyacetamide (5e)}

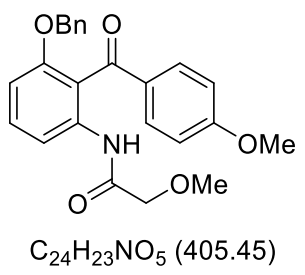

Compound 5e was prepared according to GP1 starting from $\mathbf{3 b}(571 \mathrm{mg}, 1.41 \mathrm{mmol}, 1.5$ equiv., 0.015 M) and 4b (210 mg, $0.941 \mathrm{mmol}, 1$ equiv., $0.010 \mathrm{M})$. Column chromatography (silica gel, $n$-pentane/EtOAc $=3: 1)$ afforded the title compound $(5 e, 90.6 \mathrm{mg}, 0.122 \mathrm{mmol}, 24 \%)$ as a slightly yellow oil.

$\mathbf{R}_{\mathbf{f}}=0.18(n$-pentane/EtOAc $=3: 1) ;{ }^{1} \mathrm{H}$ NMR $\left(700 \mathrm{MHz}, \mathrm{CD}_{3} \mathrm{CN}\right): \delta=8.96(\mathrm{~s}, 1 \mathrm{H}), 7.87(\mathrm{dd}, J=8.3,0.8$ $\mathrm{Hz}, 1 \mathrm{H}), 7.76-7.73(\mathrm{~m}, 2 \mathrm{H}), 7.47(\mathrm{t}, J=8.3 \mathrm{~Hz}, 1 \mathrm{H}), 7.24-7.18(\mathrm{~m}, 3 \mathrm{H}), 6.99-6.96(\mathrm{~m}, 3 \mathrm{H}), 6.95-$ $6.93(\mathrm{~m}, 2 \mathrm{H}), 4.98(\mathrm{~s}, 2 \mathrm{H}), 3.85(\mathrm{~s}, 3 \mathrm{H}), 3.84(\mathrm{~s}, 2 \mathrm{H}), 3.30(\mathrm{~s}, 3 \mathrm{H}) \mathrm{ppm} ;{ }^{13} \mathrm{C}$ NMR $\left(176 \mathrm{MHz}, \mathrm{CD}_{3} \mathrm{CN}\right)$ : $\delta=195.8,169.1,165.2,157.6,137.4,137.3,132.6,132.2,129.2,128.7,128.1,120.7,115.9,114.9$, 109.8, 72.7, 71.1, 59.7, 56.4 ppm; IR (neat): $\tilde{v}=3360,3062,3034,3009,2935,2840,1691,1645,1593$, $1509,1461,1382,1280,1254,1173,1145,1111,1070,1028,986,927,844,793,735,696,668 \mathrm{~cm}^{-1}$; HRMS (ESI): $\mathrm{m} / \mathrm{z}$ calculated for $\mathrm{C}_{24} \mathrm{H}_{23} \mathrm{NNaO}_{5}{ }^{+}\left([\mathrm{M}+\mathrm{Na}]^{+}\right)$: 428.1468; found: 428.1461 . 


\section{$N$-(2-benzoylphenyl)-2-(benzyloxy)acetamide (5f)}

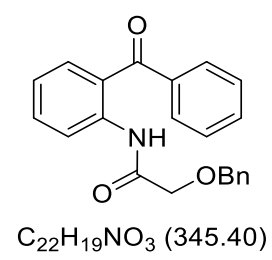

Compound $\mathbf{5 f}$ was prepared according to GP1 starting from $\mathbf{3 a}$ ( $44.8 \mathrm{mg}, 0.150 \mathrm{mmol}, 1.5$ equiv.) and 4d (29.0 mg, $0.100 \mathrm{mmol}, 1$ equiv.). Column chromatography (silica gel, $n$-pentane/EtOAc 6:1) afforded the title compound (5f, $14.1 \mathrm{mg}, 0.0404 \mathrm{mmol}, 40 \%$ ) as a colorless oil.

$\mathbf{R}_{\mathbf{f}}=0.60(n$-pentane/EtOAc $=4: 1) ;{ }^{1} \mathrm{H}$ NMR $\left(700 \mathrm{MHz}, \mathrm{CDCl}_{3}\right): \delta=11.46(\mathrm{~s}, 1 \mathrm{H}), 8.67(\mathrm{dd}, J=8.4,1.1$ $\mathrm{Hz}, 1 \mathrm{H}), 7.77-7.74(\mathrm{~m}, 2 \mathrm{H}), 7.62-7.55(\mathrm{~m}, 3 \mathrm{H}), 7.52-7.45(\mathrm{~m}, 4 \mathrm{H}), 7.37-7.33(\mathrm{~m}, 2 \mathrm{H}), 7.32-7.28$ $(\mathrm{m}, 1 \mathrm{H}), 7.15-7.10(\mathrm{~m}, 1 \mathrm{H}), 4.73(\mathrm{~s}, 2 \mathrm{H}), 4.10(\mathrm{~s}, 2 \mathrm{H}) \mathrm{ppm} ;{ }^{13} \mathrm{C} \mathrm{NMR}\left(176 \mathrm{MHz}, \mathrm{CDCl}_{3}\right): \delta=198.8$, 169.2, 139.3, 138.7, 136.9, 134.0, 133.3, 132.6, 130.2, 128.7, 128.4, 128.2, 128.1, 124.6, 122.7, 121.8, 73.8, 69.8 ppm; IR (neat): $\tilde{v}=3300,3061,3029,2955,2924,2867,1694,1641,1599,1578,1519$, 1447, 1397, 1373, 1317, 1293, 1265, 1207, 1163, 1099, 1027, 1000, 974, 936, 921, 852, 805, $75 \mathrm{~cm}^{-1}$; HRMS (ESI): $\mathrm{m} / \mathrm{z}$ calculated for $\mathrm{C}_{22} \mathrm{H}_{19} \mathrm{NNaO}_{3}{ }^{+}\left([\mathrm{M}+\mathrm{Na}]^{+}\right)$: 368.1257; found: 368.1263 .

\section{$\mathrm{N}$-(3-(allyloxy)-2-benzoylphenyl)-2-methoxyacetamide (5g)}

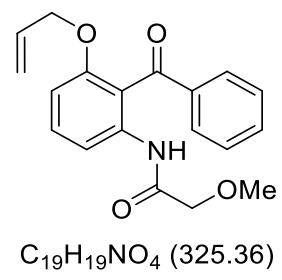

Compound $5 \mathbf{g}$ was prepared according to GP1 starting from $3 \mathbf{c}$ (107 mg, $0.303 \mathrm{mmol}, 1.5$ equiv.) and 4a $(45.0 \mathrm{mg}, 0.202 \mathrm{mmol}, 1$ equiv.). Column chromatography (silica gel, $n$-pentane/EtOAc = 3:1) afforded the title compound $(\mathbf{5 g}, 12.0 \mathrm{mg}, 0.0371 \mathrm{mmol}, 18 \%)$ as a slightly yellow oil.

$\mathbf{R}_{\mathbf{f}}=0.29\left(n\right.$-pentane/EtOAc = 3:1); ${ }^{1} \mathbf{H}$ NMR $\left(500 \mathrm{MHz}, \mathrm{CDCl}_{3}\right): \delta=9.52(\mathrm{~s}, 1 \mathrm{H}), 8.01(\mathrm{dd}, J=8.4,0.8$ $\mathrm{Hz}, 1 \mathrm{H}), 7.80-7.73(\mathrm{~m}, 2 \mathrm{H}), 7.56-7.51(\mathrm{~m}, 1 \mathrm{H}), 7.47-7.38(\mathrm{~m}, 3 \mathrm{H}), 6.73(\mathrm{dd}, J=8.4,0.9 \mathrm{~Hz}, 1 \mathrm{H})$, 5.53 (ddt, $J=17.2,10.7,4.9 \mathrm{~Hz}, 1 \mathrm{H}$ ), 4.97 (dq, $J=10.7,1.5 \mathrm{~Hz}, 1 \mathrm{H}), 4.87$ (dq, $J=17.3,1.7 \mathrm{~Hz}, 1 \mathrm{H}$ ), $4.35(\mathrm{dt}, J=5.0,1.7 \mathrm{~Hz}, 2 \mathrm{H}), 3.93(\mathrm{~s}, 2 \mathrm{H}), 3.40(\mathrm{~s}, 3 \mathrm{H}) \mathrm{ppm} ;{ }^{13} \mathrm{C} \mathrm{NMR}\left(126 \mathrm{MHz}, \mathrm{CDCl}_{3}\right): \delta=197.1$, 168.6, 157.4, 139.3, 137.1, 133.1, 132.5, 132.1, 129.3, 128.4, 118.6, 117.1, 115.1, 108.6, 72.4, 69.3, 59.6 ppm; IR (neat): $\tilde{v}=3356,3066,2951,2922,2850,1696,1648,1597,1584,1522,1468,1422$, 1370, 1362, 1314, 1277, 1197, 1178, 1145, 1114, 1076, 986, 926, 853, 808, 782, 746, 718, 702, 671, $661 \mathrm{~cm}^{-1}$; HRMS (ESI): $\mathrm{m} / \mathrm{z}$ calculated for $\left.\mathrm{C}_{19} \mathrm{H}_{19} \mathrm{NNaO}_{4}{ }^{+}(\mathrm{M}+\mathrm{Na}]^{+}\right): 348.1206$; found: 348.1207 . 


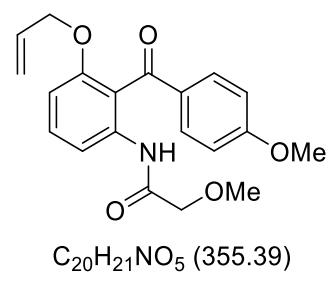

Compound $5 \mathrm{~h}$ was prepared according to GP1 starting from 3c (198 mg, $0.555 \mathrm{mmol}, 1.5$ equiv., $0.015 \mathrm{M})$ and $4 \mathrm{~b}(83.0 \mathrm{mg}, 0.370 \mathrm{mmol}, 1$ equiv., $0.010 \mathrm{M})$. Column chromatography (silica gel, $n$ pentane/EtOAc $=2: 1)$ afforded the title compound $(5 \mathrm{~h}, 39.3 \mathrm{mg}, 0.111 \mathrm{mmol}, 30 \%)$ as a slightly yellow oil.

$\mathbf{R}_{\mathbf{f}}=0.32(n$-pentane/EtOAc $=2: 1) ;{ }^{1} \mathbf{H}$ NMR $\left(500 \mathrm{MHz}, \mathrm{CDCl}_{3}\right): \delta=9.25(\mathrm{~s}, 1 \mathrm{H}), 7.95(\mathrm{~d}, J=7.8 \mathrm{~Hz}$, $1 \mathrm{H}), 7.78(\mathrm{~d}, J=8.5 \mathrm{~Hz}, 2 \mathrm{H}), 7.40(\mathrm{t}, J=8.3 \mathrm{~Hz}, 1 \mathrm{H}), 6.89(\mathrm{~d}, J=8.2 \mathrm{~Hz}, 2 \mathrm{H}), 6.74(\mathrm{~d}, J=9.1 \mathrm{~Hz}, 1 \mathrm{H})$, 5.65 (ddt, $J=17.2,10.6,4.9 \mathrm{~Hz}, 1 \mathrm{H}), 5.02(\mathrm{dq}, J=10.7,1.5 \mathrm{~Hz}, 1 \mathrm{H}), 4.96(\mathrm{dq}, J=17.3,1.7 \mathrm{~Hz}, 1 \mathrm{H}$ ), 4.40 (dt, $J=4.8,1.7 \mathrm{~Hz}, 2 \mathrm{H}), 3.90(\mathrm{~s}, 2 \mathrm{H}), 3.86(\mathrm{~s}, 3 \mathrm{H}), 3.37$ (s, 3H) ppm; ${ }^{13} \mathrm{C} \mathrm{NMR}\left(126 \mathrm{MHz}, \mathrm{CDCl}_{3}\right)$ : $\delta=195.1,168.5,164.0,156.8,136.5,132.4,132.0,131.8,131.6,119.5,117.1,115.2,113.7,108.7$, 72.3, 69.3, 59.6, 55.7 ppm; IR (neat): $\tilde{v}=3359,3075,2931,2840,1810,1693,1646,1594,1522,1509$, 1467, 1421, 1382, 1362, 1315, 1281, 1256, 1196, 1173, 1146, 1113, 1073, 1026, 986, 929, 880, 846, 817, 792, 768, 734, 709, 693, 672, $663 \mathrm{~cm}^{-1}$; HRMS (ESI): $\mathrm{m} / \mathrm{z}$ calculated for $\mathrm{C}_{20} \mathrm{H}_{21} \mathrm{NNaO}_{5}{ }^{+}\left([\mathrm{M}+\mathrm{Na}]^{+}\right)$: 378.1312; found: 378.1331 . 


\section{General Procedure for the Aldol Reaction (GP2)}

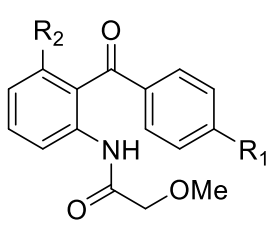

$\underset{\mathrm{THF}, 0{ }^{\circ} \mathrm{C}, 1.5 \mathrm{~h}}{\stackrel{\mathrm{KO}}{\mathrm{t} B u} \text { (7 equiv.) }}$<smiles>[R]c1ccc(C2(O)c3c([R])cccc3NC(=O)C2OC)cc1</smiles>

The ortho-aminobenzophenone (5a-h, 1 equiv.) was dissolved in anhydrous THF $(50 \mathrm{~mL} / \mathrm{mmol}$ substrate) and a solution of KO'Bu (7 equiv., $1 \mathrm{M}$ in THF) was added dropwise at $0{ }^{\circ} \mathrm{C}$. Upon addition of $\mathrm{KO} B \mathrm{Bu}$ the solution turned bright yellow. After stirring at $0{ }^{\circ} \mathrm{C}$ for $1.5 \mathrm{~h}$, water $(50 \mathrm{~mL} / \mathrm{mmol}$ substrate) was added to the reaction mixture. The organic layer was separated and the aqueous layer was extracted with EtOAc ( $3 \times 50 \mathrm{~mL} / \mathrm{mmol}$ substrate). The combined organic layers were dried $\left(\mathrm{MgSO}_{4}\right)$, filtered and concentrated under reduced pressure. The crude product was purified by column chromatography to afford the quinolinones 6a-g.

( \pm )-6-Deoxyaflaquinolone E (6a)

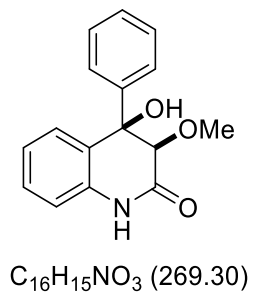

6-Deoxyaflaquinolone $E$ was prepared according to GP2 starting from $5 \mathbf{a}$ ( $20 \mathrm{mg}, 0.075 \mathrm{mmol}, 1$ equiv.) and KOtBu (59 mg, $0.53 \mathrm{mmol}, 7$ equiv.). The crude product was purified by column chromatography (silica gel, $\left.\mathrm{CH}_{2} \mathrm{Cl}_{2} / \mathrm{MeOH}=100: 3\right)$ to afford the title compound $(6 \mathbf{a}, 18 \mathrm{mg}, 0.068 \mathrm{mmol}, 92 \%$ ) as a colorless solid.

$\mathbf{R}_{\mathbf{f}}=0.21$ (n-pentane/EtOAc $\left.=1: 1\right)$; m.p.: $160{ }^{\circ} \mathrm{C}-165{ }^{\circ} \mathrm{C} ;{ }^{1} \mathbf{H}$ NMR $\left(700 \mathrm{MHz}\right.$, DMSO- $\left.d_{6}\right): \delta=10.21(\mathrm{~s}$, $1 \mathrm{H}), 7.33(\mathrm{~d}, J=4.4 \mathrm{~Hz}, 4 \mathrm{H}), 7.28(\mathrm{dt}, J=8.5,4.2 \mathrm{~Hz}, 1 \mathrm{H}), 7.24-7.20(\mathrm{~m}, 1 \mathrm{H}), 6.93-6.90(\mathrm{~m}, 2 \mathrm{H})$, $6.88(\mathrm{dd}, J=7.5,1.6 \mathrm{~Hz}, 1 \mathrm{H}), 5.81(\mathrm{~s}, 1 \mathrm{H}), 4.18(\mathrm{~s}, 1 \mathrm{H}), 3.34(\mathrm{~s}, 3 \mathrm{H}) \mathrm{ppm} ;{ }^{13} \mathrm{C}$ NMR $(176 \mathrm{MHz}$, DMSO- $\left.d_{6}\right): \delta=168.4,142.3,136.8,129.2,128.7,127.8,127.5,127.3,126.7,122.1,115.1,83.9,76.7$, 59.1 ppm; IR (neat): $\tilde{v}=3446,3361,3211,3085,3022,2993,2931,2845,2831,1681,1609,1593$, 1559, 1486, 1446, 1433, 1397, 1318, 1285, 1263, 1246, 1226, 1203, 1181, 1157, 1142, 1119, 1097, 1069, 1048, 1020, 1001, 953, 940, 910, 871, 858, 824, 791, 762, 752, 704, 674, $658 \mathrm{~cm}^{-1}$; HRMS (ESI): $\mathrm{m} / \mathrm{z}$ calculated for $\mathrm{C}_{16} \mathrm{H}_{15} \mathrm{NNaO}_{3}{ }^{+}\left([\mathrm{M}+\mathrm{Na}]^{+}\right): 292.0944$; found: 292.0945 . The NMR data match those reported for 6-deoxyaflaquinolone E. ${ }^{5}$ 


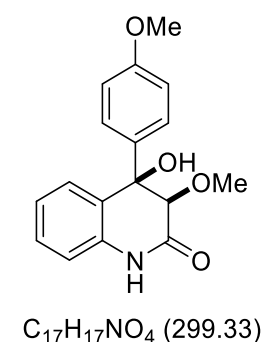

Quinolinone A was prepared according to GP2 starting from 5b (20 mg, 0.067 mmol, 1 equiv.) and $\mathrm{KO}$ 'Bu (53 mg, $0.47 \mathrm{mmol}, 7$ equiv.). The crude product was purified by column chromatography (silica gel, $\mathrm{CH}_{2} \mathrm{Cl}_{2} / \mathrm{MeOH}=100: 3$ ) to afford the title compound ( $6 \mathrm{~b}, 16.7 \mathrm{mg}, 0.056 \mathrm{mmol}, 84 \%$ ) as a colorless solid.

$\mathbf{R}_{\mathbf{f}}=0.22(n$-pentane/EtOAc $=1: 1)$; m.p.: $172{ }^{\circ} \mathrm{C}-175^{\circ} \mathrm{C} ;{ }^{1} \mathbf{H}$ NMR $\left(500 \mathrm{MHz}\right.$, acetone- $\left.d_{6}\right): \delta=9.29(\mathbf{s}$, $1 \mathrm{H}), 7.35-7.20(\mathrm{~m}, 4 \mathrm{H}), 7.07-6.97(\mathrm{~m}, 2 \mathrm{H}), 6.91-6.81(\mathrm{~m}, 2 \mathrm{H}), 4.61(\mathrm{~s}, 1 \mathrm{H}), 3.93(\mathrm{~s}, 1 \mathrm{H}), 3.76(\mathrm{~s}$, $3 \mathrm{H}), 3.45(\mathrm{~s}, 3 \mathrm{H}) \mathrm{ppm} ;{ }^{13} \mathrm{C}$ NMR $\left(126 \mathrm{MHz}\right.$, acetone- $\left.d_{6}\right): \delta=168.2,160.3,137.6,134.5,129.6,129.0$, 128.4, 123.6, 115.9, 115.9, 114.2, 85.7, 77.3, 59.4, 55.5 ppm; IR (neat): $\tilde{v}=3249,3081,3002,2931$, 2836, 1687, 1611, 1595, 1512, 1483, 1463, 1379, 1306, 1252, 1173, 1146, 1106, 1081, 1033, 991, 942 , 903, 860, 833, 812, $757 \mathrm{~cm}^{-1}$; HRMS (ESI): $\mathrm{m} / \mathrm{z}$ calculated for $\mathrm{C}_{17} \mathrm{H}_{17} \mathrm{NNaO}_{4}{ }^{+}\left([\mathrm{M}+\mathrm{Na}]^{+}\right): 322.1050$; found: 322.1047. The NMR data match those reported for Quinolinone A. ${ }^{6}$

cis-4-(4-bromophenyl)-4-hydroxy-3-methoxy-3,4-dihydroquinolin-2(1H)-one (6c)

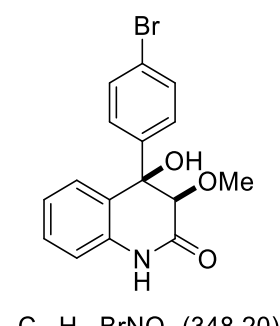

Compound $6 \mathbf{c}$ was prepared according to GP2 starting from $5 \mathbf{c}(8.0 \mathrm{mg}, 0.021 \mathrm{mmol}, 1$ equiv.) and $\mathrm{KO} \mathrm{Bu}$ (16 mg, $0.15 \mathrm{mmol}, 7$ equiv.). The crude product was purified by column chromatography (silica gel, $\mathrm{CH}_{2} \mathrm{Cl}_{2} / \mathrm{MeOH}=100: 3$ ) to afford the title compound (6c, $7.2 \mathrm{mg}, 0.019 \mathrm{mmol}, 90 \%$ ) as a colorless oil.

$\mathbf{R}_{\mathbf{f}}=0.21$ (n-pentane/EtOAc = 1:1); ${ }^{1} \mathbf{H}$ NMR $\left(700 \mathrm{MHz}, \mathrm{CD}_{3} \mathrm{OD}\right): \delta=7.51(\mathrm{~d}, \mathrm{~J}=8.8 \mathrm{~Hz}, 2 \mathrm{H}), 7.36-$ $7.33(\mathrm{~m}, 2 \mathrm{H}$ ), 7.28 (ddd, $J=8.0,5.8,3.0 \mathrm{~Hz}, 1 \mathrm{H}$ ), $7.00-6.98(\mathrm{~m}, 2 \mathrm{H}), 6.95$ (dt, $J=7.9,0.8 \mathrm{~Hz}, 1 \mathrm{H}$ ), $4.23(\mathrm{~s}, 1 \mathrm{H}), 3.40(\mathrm{~s}, 3 \mathrm{H}) \mathrm{ppm} ;{ }^{13} \mathrm{C}$ NMR $\left(176 \mathrm{MHz}, \mathrm{CDCl}_{3}\right): \delta=170.9,142.4,138.0,132.2,130.5,130.2$, 129.7, 129.1, 124.3, 122.7, 116.8, 85.4, 78.4, 60.6 ppm; IR (neat): $\tilde{v}=3241,3069,2954,2927,2853$, 2358, 1685, 1607, 1592, 1488, 1467, 1394, 1309, 1205, 1173, 1143, 989, 802, $759 \mathrm{~cm}^{-1}$; HRMS (ESI): $\mathrm{m} / \mathrm{z}$ calculated for $\mathrm{C}_{16} \mathrm{H}_{14} \mathrm{BrNO}_{3} \mathrm{Na}^{+}\left([\mathrm{M}+\mathrm{Na}]^{+}\right)$: 370.0049 ; found: 370.0051 . 


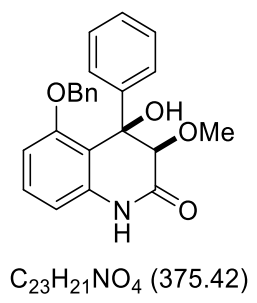

Compound 6d was prepared according to GP2 starting from $\mathbf{5 d}$ ( $28 \mathrm{mg}, 0.075 \mathrm{mmol}, 1$ equiv.) and $\mathrm{KO} B \mathrm{Bu}$ (59 mg, $0.53 \mathrm{mmol}, 7$ equiv.). The crude product was purified by column chromatography (silica gel, $\mathrm{CH}_{2} \mathrm{Cl}_{2} / \mathrm{MeOH}=100: 2$ ) to afford the title compound ( $6 \mathrm{~d}, 23 \mathrm{mg}, 0.062 \mathrm{mmol}, 84 \%$ ) as a colorless oil.

$\mathbf{R}_{\mathbf{f}}=0.25\left(n\right.$-pentane/EtOAc = 1:1); ${ }^{1} \mathbf{H}$ NMR $\left(700 \mathrm{MHz}, \mathrm{CDCl}_{3}\right): \delta=8.58(\mathrm{~s}, 1 \mathrm{H}), 7.28-7.21(\mathrm{~m}, 5 \mathrm{H})$, 7.07 (dd, $J=7.6,1.9 \mathrm{~Hz}, 2 \mathrm{H}), 6.73(\mathrm{dd}, J=8.5,0.9 \mathrm{~Hz}, 1 \mathrm{H}), 6.57(\mathrm{dd}, J=8.0,0.9 \mathrm{~Hz}, 1 \mathrm{H}), 5.29(\mathrm{~s}, 1 \mathrm{H})$, $5.05(\mathrm{~d}, J=11.5 \mathrm{~Hz}, 1 \mathrm{H}), 4.96(\mathrm{~d}, J=11.6 \mathrm{~Hz}, 1 \mathrm{H}), 3.85(\mathrm{~d}, J=1.4 \mathrm{~Hz}, 1 \mathrm{H}), 3.61(\mathrm{~s}, 3 \mathrm{H}) \mathrm{ppm} ;{ }^{13} \mathrm{C} \mathrm{NMR}$ $\left(176 \mathrm{MHz}, \mathrm{CDCl}_{3}\right): \delta=168.0,158.0,142.0,137.2,135.7,130.1,128.7,128.7,128.4,128.3,127.5$, 126.2, 115.2, 109.6, 108.8, 85.0, 78.4, 71.1, 59.8 ppm; IR (neat): $\tilde{v}=3503,3232,3062,3031,2930$, 2829, 2248, 1691, 1595, 1498, 1471, 1448, 1383, 1315, 1277, 1259, 1222, 1176, 1138, 1102, 1059, $1028,993,910,846,783,730,697,654 \mathrm{~cm}^{-1} ;$ HRMS (ESI): $\mathrm{m} / \mathrm{z}$ calculated for $\mathrm{C}_{23} \mathrm{H}_{21} \mathrm{NNaO}_{4}+\left([\mathrm{M}+\mathrm{Na}]^{+}\right)$: 398.1363; found: 398.1368 .

cis-5-(benzyloxy)-4-hydroxy-3-methoxy-4-(4-methoxyphenyl)-3,4-dihydroquinolin-2(1 H)-one (6e)

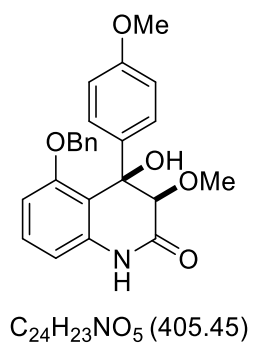

Compound 6e was prepared according to GP2 starting from 5 e (30 mg, $0.080 \mathrm{mmol}, 1$ equiv.) and $\mathrm{KO} B \mathrm{Bu}$ (63 mg, $0.56 \mathrm{mmol}, 7$ equiv.). The crude product was purified by column chromatography (silica gel, $\mathrm{CH}_{2} \mathrm{Cl}_{2} / \mathrm{MeOH}=100: 3$ ) to afford the title compound ( $6 \mathrm{e}, 25 \mathrm{mg}, 0.067 \mathrm{mmol}, 84 \%$ ) as a colorless oil.

$\mathbf{R}_{\mathbf{f}}=0.25(n$-pentane/EtOAc $=1: 1) ;{ }^{1} \mathbf{H} \mathbf{~ N M R}\left(700 \mathrm{MHz}, \mathrm{CDCl}_{3}\right): \delta=8.33(\mathrm{~s}, 1 \mathrm{H}), 7.29-7.23(\mathrm{~m}, 3 \mathrm{H})$, 7.21 (t, $J=7.8 \mathrm{~Hz}, 1 \mathrm{H}$ ), 7.16 (d, $J=7.5 \mathrm{~Hz}, 2 \mathrm{H}), 7.11-7.08(\mathrm{~m}, 2 \mathrm{H}), 6.79$ (d, $J=7.5 \mathrm{~Hz}, 2 \mathrm{H}), 6.71$ (d, $J=8.4 \mathrm{~Hz}, 1 \mathrm{H}), 6.52(\mathrm{~d}, J=8.0 \mathrm{~Hz}, 1 \mathrm{H}), 5.28(\mathrm{~s}, 1 \mathrm{H}), 5.05(\mathrm{~d}, J=11.5 \mathrm{~Hz}, 1 \mathrm{H}), 4.97(\mathrm{~d}, J=11.5 \mathrm{~Hz}$, $1 \mathrm{H}), 3.80(\mathrm{~s}, 1 \mathrm{H}), 3.76(\mathrm{~s}, 3 \mathrm{H}), 3.59(\mathrm{~s}, 3 \mathrm{H}) \mathrm{ppm} ;{ }^{13} \mathrm{C} \mathrm{NMR}\left(176 \mathrm{MHz}, \mathrm{CDCl}_{3}\right): \delta=168.0,159.7,158.1$, 137.1, 135.7, 133.6, 129.9, 128.8, 128.4, 127.6, 127.5, 115.4, 114.1, 109.5, 108.8, 85.2, 78.2, 71.1, 59.7, 55.4 ppm; IR (neat): $\tilde{v}=3502,3237,3066,3034,2999,2930,2834,1693,1596,1508,1471$, 1388, 1302, 1280, 1253, 1231, 1172, 1103, 1061, 1031, 993, 913, 895, 834, 778, 734, 698, $656 \mathrm{~cm}^{-1}$; HRMS (ESI): $\mathrm{m} / \mathrm{z}$ calculated for $\mathrm{C}_{24} \mathrm{H}_{23} \mathrm{NNaO}_{5}{ }^{+}\left([\mathrm{M}+\mathrm{Na}]^{+}\right): 428.1468$; found: 428.1467 . 


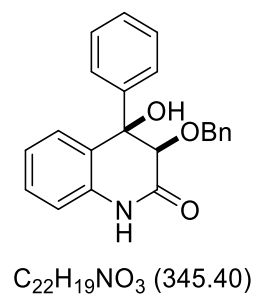

Compound $\mathbf{6 f}$ was prepared according to GP2 starting from $\mathbf{5 f}$ ( $14 \mathrm{mg}, 0.043 \mathrm{mmol}, 1$ equiv.) and KOtBu (34 $\mathrm{mg}, 0.30 \mathrm{mmol}, 7$ equiv.). The crude product was purified by column chromatography (silica gel, $\left.\mathrm{CH}_{2} \mathrm{Cl}_{2} / \mathrm{MeOH}=100: 3\right)$ to afford the title compound $(6 \mathrm{f}, 12 \mathrm{mg}, 0.031 \mathrm{mmol}, 72 \%)$ as a colorless oil.

$\mathbf{R}_{\mathbf{f}}=0.23\left(\right.$ Pentane/EtOAc = 1:1); ${ }^{1} \mathbf{H}$ NMR $\left(500 \mathrm{MHz}, \mathrm{CD}_{3} \mathrm{OD}\right): \delta=7.40-7.32(\mathrm{~m}, 5 \mathrm{H}), 7.27$ (ddd, $J=$ 7.9, 6.3, 2.6 Hz, 1H), $7.20-7.15(\mathrm{~m}, 3 \mathrm{H}), 7.03-6.99(\mathrm{~m}, 2 \mathrm{H}), 6.99-6.93(\mathrm{~m}, 3 \mathrm{H}), 4.81(\mathrm{~d}, J=11.5$ $\mathrm{Hz}, 1 \mathrm{H}), 4.50(\mathrm{~s}, 1 \mathrm{H}), 4.45$ (d, $J=11.5 \mathrm{~Hz}, 1 \mathrm{H}) \mathrm{ppm} ;{ }^{13} \mathrm{C} \mathbf{N M R}\left(176 \mathrm{MHz}, \mathrm{CDCl}_{3}\right): \delta=168.5,140.7$, 136.7, 135.7, 129.7, 128.8, 128.6, 128.6, 128.5, 128.5, 128.2, 128.1, 126.8, 124.1, 115.5, 81.1, 73.8 ppm; IR (neat): $\tilde{v}=3263,3087,3060,3029,2923,2854,1692,1610,1595,1485,1448,1375,1296$, $1265,1241,1211,1174,1143,1125,1096,1070,1044,1028,989,936,907,853,811,753 \mathrm{~cm}^{-1}$; HRMS (ESI): $\mathrm{m} / \mathrm{z}$ calculated for $\mathrm{C}_{22} \mathrm{H}_{19} \mathrm{NO}_{3} \mathrm{~K}^{+}\left([\mathrm{M}+\mathrm{K}]^{+}\right)$: 384.0997; found: 384.1002 .

cis-5-(allyloxy)-4-hydroxy-3-methoxy-4-phenyl-3,4-dihydroquinolin-2(1H)-one (6g)

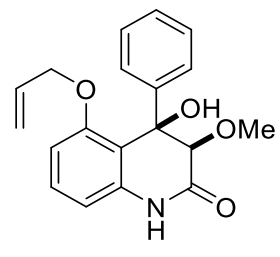

$\mathrm{C}_{19} \mathrm{H}_{19} \mathrm{NO}_{4}(325.36)$

Compound $\mathbf{6 g}$ was prepared according to GP2 starting from $\mathbf{5 g}(35 \mathrm{mg}, 0.11 \mathrm{mmol}, 1$ equiv.) and KOtBu (86 mg, $0.77 \mathrm{mmol}, 7$ equiv.). The crude product was purified by column chromatography (silica gel, $\left.\mathrm{CH}_{2} \mathrm{Cl}_{2} / \mathrm{MeOH}=100: 2\right)$ to afford the title compound $(6 \mathrm{~g}, 27 \mathrm{mg}, 0.072 \mathrm{mmol}, 67 \%$ ) as a colorless oil.

$\mathbf{R}_{\mathbf{f}}=0.23$ (n-pentane/EtOAc = 1:1); ${ }^{1} \mathrm{H}$ NMR $\left(500 \mathrm{MHz}, \mathrm{CDCl}_{3}\right): \delta=8.07(\mathrm{~s}, 1 \mathrm{H}), 7.29-7.26(\mathrm{~m}, 5 \mathrm{H})$, 7.22 (t, $J=8.2 \mathrm{~Hz}, 1 \mathrm{H}$ ), 6.65 (dd, $J=8.4,1.0 \mathrm{~Hz}, 1 \mathrm{H}$ ), 6.52 (dd, $J=8.0,0.9 \mathrm{~Hz}, 1 \mathrm{H}$ ), 5.78 (ddt, $J=17.2$, 10.6, $5.4 \mathrm{~Hz}, 1 \mathrm{H}), 5.34(\mathrm{~s}, 1 \mathrm{H}), 5.21-5.15(\mathrm{~m}, 2 \mathrm{H}), 4.52-4.41(\mathrm{~m}, 2 \mathrm{H}), 3.84(\mathrm{~d}, J=1.4 \mathrm{~Hz}, 1 \mathrm{H}), 3.58$ (s, 3H) ppm; ${ }^{13} \mathrm{C}$ NMR $\left(126 \mathrm{MHz}, \mathrm{CDCl}_{3}\right): \delta=167.7,158.0,141.9,137.2,132.1,130.0,128.7,128.5$, 126.1, 118.8, 115.0, 109.4, 108.7, 85.1, 78.4, 69.8, 59.8 ppm; IR (neat): $\tilde{v}=3494,3249,3235,3083$, 3002, 2928, 2829, 1692, 1596, 1502, 1473, 1447, 1421, 1391, 1324, 1313, 1278, 1259, 1224, 1179, 1103, 1059, 1028, 992, 930, 887, 824, 784, 750, 731, 699, 674, 665, $654 \mathrm{~cm}^{-1} ;$ HRMS (ESI): m/z calculated for $\mathrm{C}_{19} \mathrm{H}_{19} \mathrm{NO}_{4} \mathrm{Na}^{+}\left([\mathrm{M}+\mathrm{Na}]^{+}\right): 348.1206$; found: 348.1195 . 


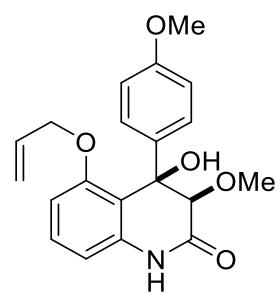

$\mathrm{C}_{20} \mathrm{H}_{21} \mathrm{NO}_{5}$ (355.39)

Compound $6 \mathrm{~h}$ was prepared according to GP2 starting from $5 \mathrm{~h}$ ( $43 \mathrm{mg}, 0.12 \mathrm{mmol}, 1$ equiv.) and KOtBu (94 mg, $0.84 \mathrm{mmol}, 7$ equiv.). The crude product was purified by column chromatography (silica gel $\left.\mathrm{CH}_{2} \mathrm{Cl}_{2} / \mathrm{MeOH}=100: 3\right)$ to afford the title compound ( $\left.6 \mathbf{h}, 25 \mathrm{mg}, 0.070 \mathrm{mmol}, 58 \%\right)$ as a colorless oil.

$\mathbf{R}_{\mathbf{f}}=0.23(n$-pentane/EtOAc $=1: 1) ;{ }^{1} \mathbf{H}$ NMR $\left(500 \mathrm{MHz}, \mathrm{CDCl}_{3}\right): \delta=8.34(\mathrm{~s}, 1 \mathrm{H}), 7.21(\mathrm{t}, J=8.2 \mathrm{~Hz}, 1 \mathrm{H})$, $7.18-7.15(\mathrm{~m}, 2 \mathrm{H}), 6.81-6.77(\mathrm{~m}, 2 \mathrm{H}), 6.65(\mathrm{~d}, J=8.5 \mathrm{~Hz}, 1 \mathrm{H}), 6.51(\mathrm{dd}, J=8.0,0.8 \mathrm{~Hz}, 1 \mathrm{H}), 5.83$ (ddt, $J=17.0,10.4,5.3 \mathrm{~Hz}, 1 \mathrm{H}), 5.40(\mathrm{~s}, 1 \mathrm{H}), 5.25-5.16(\mathrm{~m}, 2 \mathrm{H}), 4.54-4.44(\mathrm{~m}, 2 \mathrm{H}), 3.81(\mathrm{~d}, J=1.5$ $\mathrm{Hz}, 1 \mathrm{H}), 3.74(\mathrm{~s}, 3 \mathrm{H}), 3.59$ (s, 3H) ppm; ${ }^{13} \mathrm{C}$ NMR $\left(126 \mathrm{MHz}, \mathrm{CDCl}_{3}\right): \delta=167.9,159.7,158.0,137.0$, 133.6, 132.1 1 129.8, 127.5, 118.8, 115.2, 114.1, 109.5, 108.7, 85.2, 78.2, 69.8, 59.6, 55.3 ppm; IR (neat): $\tilde{v}=3697,3251,3237,3196,3101,3087$ 3039, 2997, 2932, 2833, 2246, 1691, 1595, 1508, 1471, 1442, $1417,1392,1303,1280,1251,1224,1172,1101,993,928,912,893,833,809,779,729,693,670$, $661 \mathrm{~cm}^{-1} ;$ HRMS (ESI): $\mathrm{m} / \mathrm{z}$ calculated for $\mathrm{C}_{20} \mathrm{H}_{21} \mathrm{NNaO}_{5}+\left([\mathrm{M}+\mathrm{Na}]^{+}\right): 378.1312$; found: 378.1317 . 
( \pm -Aflaquinolone $E(9)$

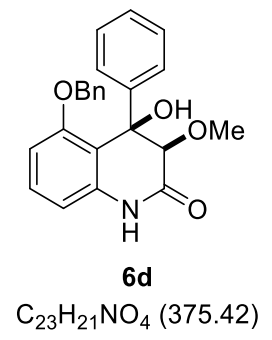

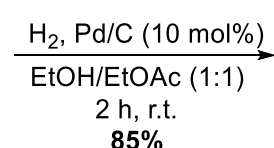

$85 \%$

$\mathrm{C}_{23} \mathrm{H}_{21} \mathrm{NO}_{4}$ (375.42)

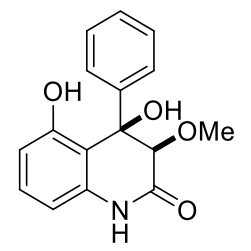

9

$\mathrm{C}_{16} \mathrm{H}_{15} \mathrm{NO}_{4}(285.30)$

To a solution of $6 \mathbf{d}(23 \mathrm{mg}, 0.060 \mathrm{mmol}, 1$ equiv.) EtOH/EtOAc (1:1, $1 \mathrm{~mL})$ was added $\mathrm{Pd} / \mathrm{C}(6.4 \mathrm{mg}$, $0.0061 \mathrm{mmol}, 10 \mathrm{w} \% \mathrm{Pd}, 10 \mathrm{~mol} \%$ ) and the atmosphere was changed from $\mathrm{Ar}$ to $\mathrm{H}_{2}$ by short evacuation and backfilling with $\mathrm{H}_{2}$. The reaction mixture was then stirred vigorously at room temperature for $2 \mathrm{~h}$ under an atmosphere of $\mathrm{H}_{2}$. Then the reaction mixture was then filtered over a short pad of Celite ${ }^{\circledR}$ and concentrated under reduced pressure. The crude product was purified by column chromatography (silica gel, $\left.\mathrm{CH}_{2} \mathrm{Cl}_{2} / \mathrm{MeOH}=50: 1\right)$ to afford the title compound $(9,15 \mathrm{mg}, 0.051 \mathrm{mmol}, 85 \%)$ as a colorless solid.

$\mathbf{R}_{\mathbf{f}}=0.21$ (n-pentane/EtOAC $=3: 1$ ); $\mathbf{m} . \mathbf{p .}: 143^{\circ} \mathrm{C}-148{ }^{\circ} \mathrm{C} ;{ }^{1} \mathbf{H}$ NMR $\left(700 \mathrm{MHz}, \mathrm{CD}_{3} \mathrm{OD}\right): \delta=7.32-7.29$ (m, 3H), $7.29-7.26(\mathrm{~m}, 2 \mathrm{H}), 7.15(\mathrm{t}, J=8.1 \mathrm{~Hz}, 1 \mathrm{H}), 6.54(\mathrm{dd}, J=8.3,1.1 \mathrm{~Hz}, 1 \mathrm{H}), 6.46(\mathrm{dd}, J=8.0$, $1.1 \mathrm{~Hz}, 1 \mathrm{H}), 3.66(\mathrm{~s}, 1 \mathrm{H}), 3.53(\mathrm{~s}, 3 \mathrm{H}) \mathrm{ppm} ;{ }^{13} \mathrm{C}$ NMR (176 MHz, $\left.\mathrm{CD}_{3} \mathrm{OD}\right): \delta=169.1,159.1,140.9,138.1$, 131.0, 129.8, 129.6, 127.5, 113.2, 113.0, 108.2, 86.3, 79.9, 59.2 ppm; IR (neat): $\tilde{v}=3281,3061,2998$, 2935, 2832, 1681, 1620, 1595, 1475, 1448, 1387, 1243, 1207, 1169, 1099, 1021, 879, 791, 745, 725, 698, $659 \mathrm{~cm}^{-1}$; HRMS (ESI): m/z calculated for $\mathrm{C}_{16} \mathrm{H}_{15} \mathrm{NNaO}_{4}{ }^{+}\left([\mathrm{M}+\mathrm{Na}]^{+}\right): 308.0893$; found: 308.0900 . The NMR data match those reported for Aflaquinolone E. ${ }^{7}$

\section{(士)-Quinolinone B (10)}

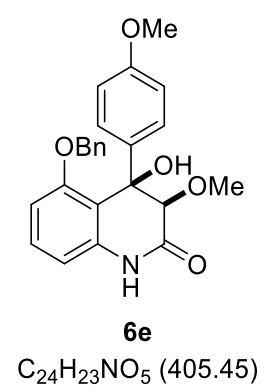

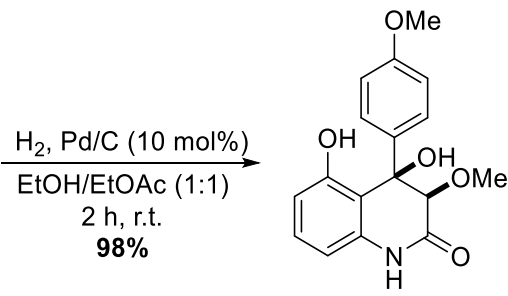

10

$\mathrm{C}_{17} \mathrm{H}_{17} \mathrm{NO}_{5}(315.33)$

To a solution of $6 \mathrm{e}(12 \mathrm{mg}, 0.028 \mathrm{mmol}, 1$ equiv.) in $\mathrm{EtOH} / \mathrm{EtOAc}(1: 1,1 \mathrm{~mL})$ was added $\mathrm{Pd} / \mathrm{C}(3.0 \mathrm{mg}$, $0.0029 \mathrm{mmol}, 10 \mathrm{w} \% \mathrm{Pd}, 10 \mathrm{~mol} \%$ ) and the atmosphere was changed from $\mathrm{Ar}$ to $\mathrm{H}_{2}$ by short evacuation and backfilling with $\mathrm{H}_{2}$. The reaction mixture was then stirred vigorously at room temperature for $2 \mathrm{~h}$ under an atmosphere of $\mathrm{H}_{2}$. The reaction mixture was then filtered over a short pad of Celite ${ }^{\circledR}$ and concentrated under reduced pressure. The crude product was purified by column chromatography (silica gel $\left.\mathrm{CH}_{2} \mathrm{Cl}_{2} / \mathrm{MeOH}=50: 1\right)$ to afford the title compound $(10,8.8 \mathrm{mg}, 0.028 \mathrm{mmol}, 98 \%)$ as a colorless oil.

$\mathbf{R}_{\mathbf{f}}=0.60\left(\mathrm{CH}_{2} \mathrm{Cl}_{2} / \mathrm{MeOH}=50: 1\right) ;{ }^{1} \mathrm{H}$ NMR $\left(700 \mathrm{MHz}\right.$, acetone- $\left.\mathrm{d}_{6}\right): \delta=9.28(\mathrm{~s}, 1 \mathrm{H}), 9.16(\mathrm{~s}, 1 \mathrm{H}), 7.22$ $(\mathrm{d}, J=8.9 \mathrm{~Hz}, 1 \mathrm{H}), 7.16(\mathrm{t}, J=8.1 \mathrm{~Hz}, 1 \mathrm{H}), 6.88(\mathrm{~d}, J=8.9 \mathrm{~Hz}, 1 \mathrm{H}), 6.55(\mathrm{dd}, J=8.0,1.1 \mathrm{~Hz}, 1 \mathrm{H}), 6.49$ (dd, $J=8.2,1.1 \mathrm{~Hz}, 1 \mathrm{H}), 6.14(\mathrm{~s}, 1 \mathrm{H}), 3.77(\mathrm{~s}, 3 \mathrm{H}), 3.67(\mathrm{~d}, J=1.6 \mathrm{~Hz}, 1 \mathrm{H}), 3.50(\mathrm{~s}, 3 \mathrm{H}) \mathrm{ppm} ;{ }^{13} \mathrm{C} \mathrm{NMR}$ 
(176 MHz, acetone- $\left.\mathrm{d}_{6}\right): \delta=166.5,161.0,159.2,138.1,131.9,130.7,128.8,114.7,112.5,112.4,107.4$, 85.8, 79.6, 58.9, 55.5. ppm; IR (neat): $\tilde{v}=3294,3066,2993,2932,2837,1685,1626,1595,1510,1439$, 1379, 1305, 1253, 1207, 1170, 1103, 1076, 1051, 1025, 989, 936, 884, 834, 783, 756, $716 \mathrm{~cm}^{-1}$; HRMS (ESI): $\mathrm{m} / \mathrm{z}$ calculated for $\mathrm{C}_{17} \mathrm{H}_{17} \mathrm{NNaO}_{5}{ }^{+}\left([\mathrm{M}+\mathrm{Na}]^{+}\right)$: 338.0999; found: 338.1006 . The NMR data match those reported for Quinolinone B. ${ }^{6}$

( \pm )-Aflaquinolone $F(11)$
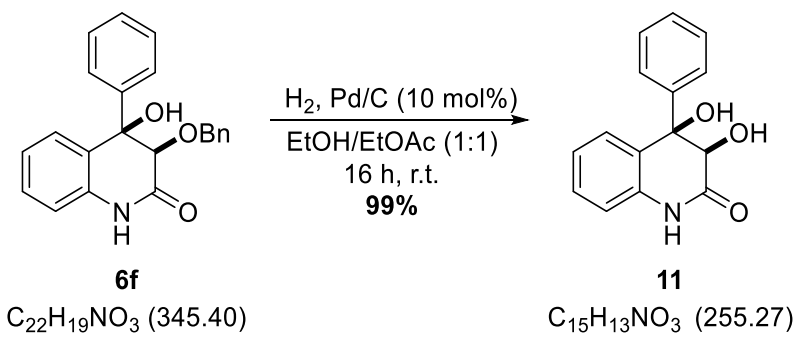

To a solution of $6 \mathbf{f}$ (12 mg, $0.034 \mathrm{mmol}, 1$ equiv.) in EtOH/EtOAc $(1: 1,1 \mathrm{~mL})$ was added $\mathrm{Pd} / \mathrm{C}(3.6 \mathrm{mg}$, $0.0031 \mathrm{mmol}, 10 \mathrm{w} \% \mathrm{Pd}, 10 \mathrm{~mol} \%$ ) and the atmosphere was changed from $\mathrm{Ar}$ to $\mathrm{H}_{2}$ by short evacuation and backfilling with $\mathrm{H}_{2}$. The reaction mixture was then stirred vigorously at room temperature for $16 \mathrm{~h}$ under an atmosphere of $\mathrm{H}_{2}$. The reaction mixture was then filtered over a short pad of Celite ${ }^{\circledR}$ and concentrated under reduced pressure. The crude product was purified by column chromatography (silica gel, $\left.\mathrm{CH}_{2} \mathrm{Cl}_{2} / \mathrm{MeOH}=100: 3\right)$ to afford the title compound $(\mathbf{1 1}, 8.7 \mathrm{mg}, 0.034 \mathrm{mmol}, 99 \%)$ as a colorless oil.

$\mathbf{R}_{\mathbf{f}}=0.30\left(\mathrm{CH}_{2} \mathrm{Cl}_{2} / \mathrm{MeOH}=50: 1\right) ;{ }^{1} \mathrm{H}$ NMR $\left(600 \mathrm{MHz}, \mathrm{CD}_{3} \mathrm{OD}\right): \delta=7.50(\mathrm{~d}, J=8.2 \mathrm{~Hz}, 2 \mathrm{H}), 7.41-7.36$ $(\mathrm{m}, 2 \mathrm{H}), 7.34-7.30(\mathrm{~m}, 1 \mathrm{H}), 7.29-7.20(\mathrm{~m}, 1 \mathrm{H}), 6.96(\mathrm{~s}, 1 \mathrm{H}), 6.98-6.85(\mathrm{~m}, 1 \mathrm{H}), 6.73-6.70(\mathrm{~m}, 1 \mathrm{H})$, $5.48(\mathrm{~s}, 1 \mathrm{H}), 4.75(\mathrm{~s}, 1 \mathrm{H}) \mathrm{ppm} ;{ }^{13} \mathrm{C}$ NMR $\left(151 \mathrm{MHz}, \mathrm{CD}_{3} \mathrm{OD}\right): \delta=172.7,143.2,138.4,130.6,130.1$, 129.0, 128.4, 128.3, 124.0, 117.0, 78.7, 75.8, 49.4 ppm; IR (neat): $\tilde{v}=3294,2954,2922,2853,1727$, 1707, 1606, 1464, 1376, 1282, 1248, 1116, 1031, 824, 762, $722 \mathrm{~cm}^{-1} ;$ HRMS (ESI): m/z calculated for $\mathrm{C}_{15} \mathrm{H}_{13} \mathrm{NNaO}_{3}{ }^{+}\left([\mathrm{M}+\mathrm{Na}]^{+}\right): 278.0787$; found: 278.0795 . The NMR data match those reported for Aflaquinolone $\mathrm{F}^{7}$

\section{cis-6-allyl-4,5-dihydroxy-3-methoxy-4-phenyl-3,4-dihydroquinolin-2(1H)-one (12a)}
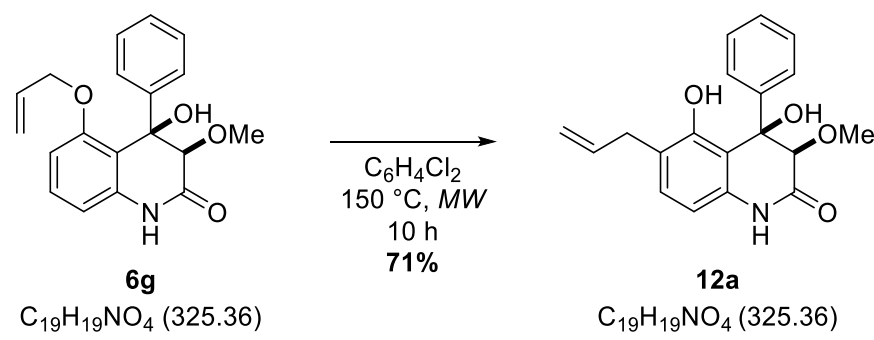

$12 \mathrm{a}$

$\mathrm{C}_{19} \mathrm{H}_{19} \mathrm{NO}_{4}(325.36)$

$6 \mathrm{~g}$ (14 mg, $0.040 \mathrm{mmol}, 1$ equiv.) was dissolved in 1,2-dichlorobenzene (1 $\mathrm{mL})$ and heated in a microwave at $150^{\circ} \mathrm{C}$ for $10 \mathrm{~h}$. The solvent was removed under reduced pressure, and the crude product was purified by column chromatography (silica gel, $n$-pentane/EtOAc $=1: 2$ ) to afford the title compound (12a, $9.9 \mathrm{mg}, 0.030 \mathrm{mmol}, 71 \%$ ) as a colorless oil. 
$\mathbf{R}_{\mathbf{f}}=0.32\left(\mathrm{CH}_{2} \mathrm{Cl}_{2} / \mathrm{MeOH}=50: 1\right) ;{ }^{1} \mathrm{H}$ NMR $\left(500 \mathrm{MHz}, \mathrm{CDCl}_{3}\right): \delta=8.90(\mathrm{~d}, J=0.5 \mathrm{~Hz}, 1 \mathrm{H}), 7.85(\mathrm{~s}, 1 \mathrm{H})$, $7.33-7.29(\mathrm{~m}, 3 \mathrm{H}), 7.28-7.25(\mathrm{~m}, 2 \mathrm{H}), 7.05(\mathrm{~d}, J=8.0 \mathrm{~Hz}, 1 \mathrm{H}), 6.32(\mathrm{~d}, J=8.0 \mathrm{~Hz}, 1 \mathrm{H}), 5.97$ (ddt, $J=16.8,10.1,6.6 \mathrm{~Hz}, 1 \mathrm{H}), 5.07-5.02(\mathrm{~m}, 2 \mathrm{H}), 4.60(\mathrm{~s}, 1 \mathrm{H}), 3.69(\mathrm{~d}, J=1.5 \mathrm{~Hz}, 1 \mathrm{H}), 3.62(\mathrm{~s}, 3 \mathrm{H}), 3.31$ (qdt, $J=15.7,6.7,1.7 \mathrm{~Hz}, 2 \mathrm{H}$ ) ppm; ${ }^{13} \mathrm{C}$ NMR $\left(126 \mathrm{MHz}, \mathrm{CDCl}_{3}\right): \delta=165.7,155.7,137.7,136.9,133.8$, $130.8,129.3,129.0,126.5,124.4,115.7,110.5,106.5,84.3,79.1,59.1,33.7$ ppm; IR (neat): $\tilde{v}=3465$, 3290, 3074, 2954, 2924, 2869, 1683, 1638, 1622, 1602, 1505, 1493, 1463, 1449, 1421, 1378, 1346, 1272, 1223, 1174, 1102, 1077, 1036, 1027, 992, 947, 916, 892, 856, 846, 815, 754, 697, 673, $666 \mathrm{~cm}^{-1}$; HRMS (ESI): $m / z$ calculated for $\mathrm{C}_{19} \mathrm{H}_{19} \mathrm{NNaO}_{4}{ }^{+}\left([\mathrm{M}+\mathrm{Na}]^{+}\right): 348.1206$; found: 348.1216 .

cis-6-allyl-4,5-dihydroxy-3-methoxy-4-(4-methoxyphenyl)-3,4-dihydroquinolin-2(1H)-one (12b)
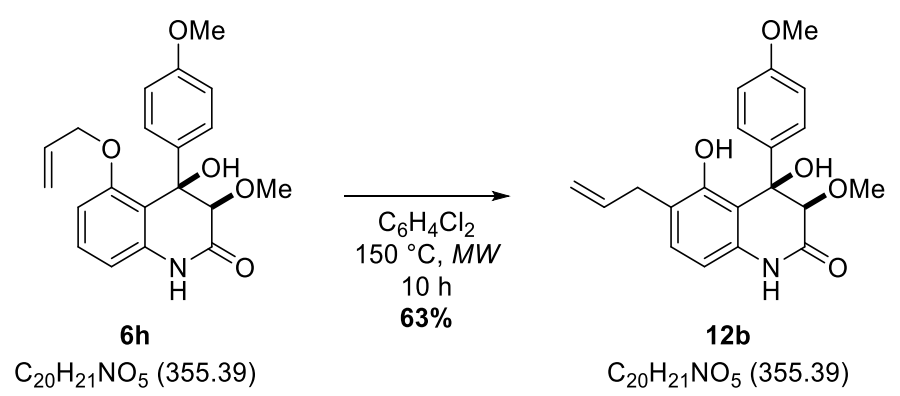

6h (39 mg, $0.11 \mathrm{mmol}, 1$ equiv.) was dissolved in 1,2-dichlorobenzene (1 mL) and heated in a microwave at $150^{\circ} \mathrm{C}$ for $10 \mathrm{~h}$. The solvent was removed under reduced pressure, and the crude product was purified by column chromatography (silica gel, $n$-pentane/EtOAc $=1: 2$ ) to afford the title compound (12b, $25 \mathrm{mg}, 0.070 \mathrm{mmol}, 63 \%$ ) as a colorless oil.

$\mathbf{R}_{\mathbf{f}}=0.32(n$-Pentane/EtOAc $=1: 1) ;{ }^{1} \mathbf{H}$ NMR $\left(400 \mathrm{MHz}\right.$, acetone- $\left.d_{6}\right): \delta=9.45(\mathrm{~s}, 1 \mathrm{H}), 9.25(\mathrm{~s}, 1 \mathrm{H}), 7.28$ $-7.15(\mathrm{~m}, 2 \mathrm{H}), 7.04(\mathrm{dt}, J=8.1,0.7 \mathrm{~Hz}, 1 \mathrm{H}), 6.93-6.82(\mathrm{~m}, 2 \mathrm{H}), 6.51$ (d, $J=8.5 \mathrm{~Hz}, 1 \mathrm{H}), 6.23(\mathrm{~s}, 1 \mathrm{H})$, $6.01-5.89(\mathrm{~m}, 1 \mathrm{H}), 5.08-5.00(\mathrm{~m}, 1 \mathrm{H}), 4.96(\mathrm{ddtd}, J=10.1,2.0,1.4,0.5 \mathrm{~Hz}, 1 \mathrm{H}), 3.76(\mathrm{~s}, 2 \mathrm{H}), 3.65$ (dd, $J=1.5,0.5 \mathrm{~Hz}, 1 \mathrm{H}), 3.50(\mathrm{~s}, 1 \mathrm{H}), 3.33-3.20(\mathrm{~m}, 2 \mathrm{H}) \mathrm{ppm} ;{ }^{13} \mathrm{C}$ NMR $\left(126 \mathrm{MHz}\right.$, acetone- $\left.d_{6}\right): \delta=$ 166.4, 161.0, 156.6, 138.1, 136.3, 131.9, 130.8, 128.8, 123.2, 115.4, 114.7, 112.0, 107.1, 85.8, 79.7, 58.8, 55.5, 34.2. ppm; IR (neat): $\tilde{v}=3280,3069,2955,2924,2853,1683,1638,1623,1603,1509$, 1463, 1418, 1379, 1306, 1253, 1224, 1172, 1103, 1077, 1028, 993, 944, 909, 891, 860, 830, 812, 798, $765,754,730,709,701,687,681,666 \mathrm{~cm}^{-1}$; HMRS (ESI): $\mathrm{m} / z$ calculated for $\mathrm{C}_{20} \mathrm{H}_{21} \mathrm{NNaO}_{5}{ }^{+}\left([\mathrm{M}+\mathrm{Na}]^{+}\right)$: 378.1312; found: 378.1328 .

\section{( \pm )-Aniduquinolone C (13a)}
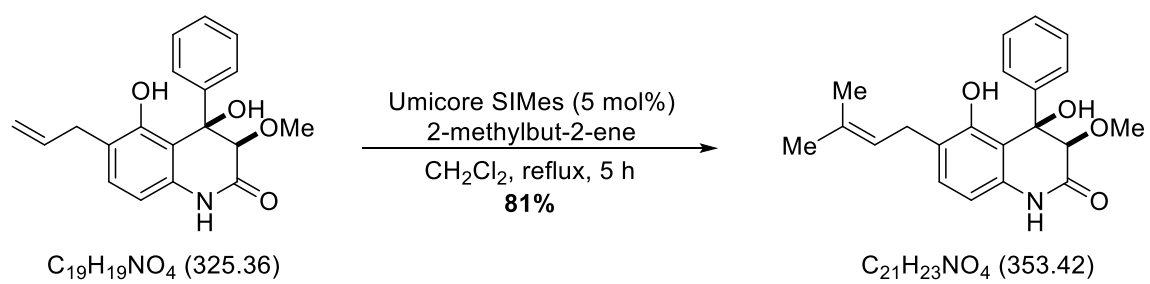

12a (9.9 mg, $0.030 \mathrm{mmol}, 1$ equiv.) and Umicore M71 SIMes (1.1 mg, $0.0015 \mathrm{mmol}, 5 \mathrm{~mol} \%$ ) were dissolved in $\mathrm{CH}_{2} \mathrm{Cl}_{2}(0.5 \mathrm{~mL})$. 2-methylbut-2-ene $(0.030 \mathrm{~mL}, 0.30 \mathrm{mmol}, 10$ equiv.) was added and the reaction mixture was heated to reflux for $5 \mathrm{~h}$ in a sealed tube. After cooling to room temperature, the 
reaction mixture was filtered through a short pad of silica gel. All volatiles were removed under reduced pressure, and the crude product was purified by $\mathrm{HPLC}\left(\mathrm{EtOH} / n\right.$-pentane $\left.=1: 10,1 \mathrm{~mL} / \mathrm{min}, \mathrm{tr}_{\mathrm{r}}=7.00 \mathrm{~min}\right)$ to afford the title compound (13a, $8.0 \mathrm{mg}, 0.024 \mathrm{mmol}, 81 \%$ ) as a colorless oil.

$\mathbf{R}_{\mathbf{f}}=0.35\left(\mathrm{CH}_{2} \mathrm{Cl}_{2} / \mathrm{MeOH} 50: 1\right) ;{ }^{1} \mathbf{H}$ NMR $\left(700 \mathrm{MHz}\right.$, DMSO- $\left.d_{6}\right): \delta=10.15(\mathrm{~s}, 1 \mathrm{H}), 7.37-7.28(\mathrm{~m}, 3 \mathrm{H})$, $7.19(\mathrm{dd}, J=5.8,3.6 \mathrm{~Hz}, 2 \mathrm{H}), 6.95(\mathrm{~d}, J=7.9 \mathrm{~Hz}, 1 \mathrm{H}), 6.37(\mathrm{~d}, J=7.9 \mathrm{~Hz}, 1 \mathrm{H}), 5.24(\mathrm{t}, J=7.4 \mathrm{~Hz}, 1 \mathrm{H})$, $3.58(\mathrm{~s}, 1 \mathrm{H}), 3.43(\mathrm{~s}, 3 \mathrm{H}), 3.19$ (dd, $J=15.4,7.4 \mathrm{~Hz}, 1 \mathrm{H}), 3.08(\mathrm{dd}, J=15.4,7.3 \mathrm{~Hz}, 1 \mathrm{H}), 1.68(\mathrm{~s}, 3 \mathrm{H})$, $1.65(\mathrm{~s}, 3 \mathrm{H}) \mathrm{ppm} ;{ }^{13} \mathrm{C}$ NMR $\left(176 \mathrm{MHz}\right.$, DMSO- $\left.d_{6}\right): \delta=166.1,155.0,140.0,135.0,131.3,129.2,128.6$, 126.2, 123.0, 122.8, 110.9, 106.3, 84.4, 78.7, 58.3, 27.5, 25.6, 17.7 ppm; IR (neat): $\tilde{v}=3218,3142$, 3062, 2912, 2832, 2255, 1685, 1623, 1602, 1506, 1493, 1447, 1422, 1375, 1274, 1224, 1174, 1105, 1076, 1024, 1003, 943, 902, 867, 818, 767, $734 \mathrm{~cm}^{-1}$; HRMS (ESI): $\mathrm{m} / z$ calculated for $\mathrm{C}_{21} \mathrm{H}_{23} \mathrm{NNaO}_{4}{ }^{+}$ $\left([\mathrm{M}+\mathrm{Na}]^{+}\right): 376.1519$; found: 376.1513 . The NMR data match those reported for Aniduquinolone $\mathrm{C} .^{5}$

\section{(士)-Peniprequinolone (13b)}
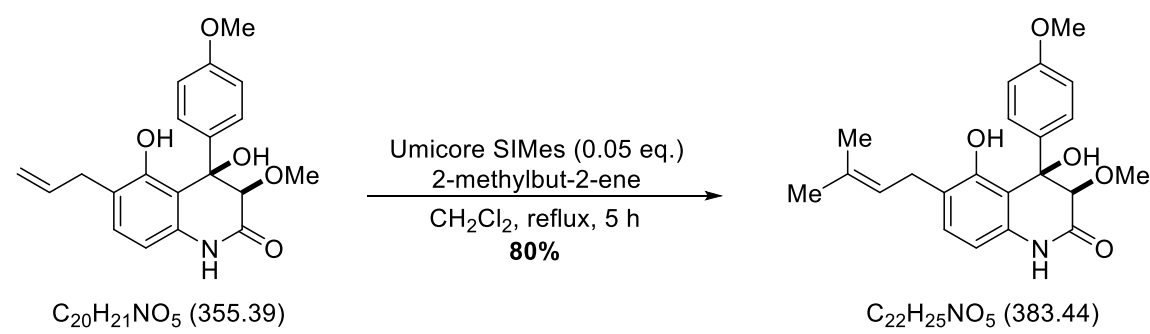

$\mathrm{C}_{22} \mathrm{H}_{25} \mathrm{NO}_{5}(383.44)$

12b (11 mg, $0.030 \mathrm{mmol}, 1$ equiv.) and Umicore M71 SIMes (1.1 mg, $0.0015 \mathrm{mmol}, 5 \mathrm{~mol} \%$ ) were dissolved in $\mathrm{CH}_{2} \mathrm{Cl}_{2}(0.5 \mathrm{~mL})$. 2-methylbut-2-ene $(0.030 \mathrm{~mL}, 0.30 \mathrm{mmol}, 10$ equiv.) was added and the reaction mixture was heated to reflux for $5 \mathrm{~h}$ in a sealed tube. After cooling to room temperature, the reaction mixture was filtered through a short pad of silica gel. All volatiles were removed under reduced pressure, and the crude product was purified by HPLC $\left(\mathrm{EtOH} / n\right.$-pentane $\left.=1: 10,1 \mathrm{~mL} / \mathrm{min}, \mathrm{t}_{\mathrm{r}}=8.60 \mathrm{~min}\right)$ to afford the title compound (13b, $9.0 \mathrm{mg}, 0.024 \mathrm{mmol}, 80 \%$ ) as a colorless solid.

$\mathbf{R}_{\mathbf{f}}=0.40\left(\mathrm{CH}_{2} \mathrm{Cl}_{2} / \mathrm{MeOH} 50: 1\right)$; m.p.: $59^{\circ} \mathrm{C} ;{ }^{1} \mathbf{H} \mathbf{~ N M R}\left(500 \mathrm{MHz}, \mathrm{CDCl}_{3}\right): \delta=8.91(\mathrm{~s}, 1 \mathrm{H}), 7.91(\mathrm{~s}, 1 \mathrm{H})$, $7.19-7.14(\mathrm{~m}, 2 \mathrm{H}), 7.03(\mathrm{~d}, \mathrm{~J}=8.0 \mathrm{~Hz}, 1 \mathrm{H}), 6.84-6.78(\mathrm{~m}, 2 \mathrm{H}), 6.28(\mathrm{~d}, \mathrm{~J}=8.0 \mathrm{~Hz}, 1 \mathrm{H}), 5.30-5.26$ $(\mathrm{m}, 1 \mathrm{H}), 4.54(\mathrm{~s}, 1 \mathrm{H}), 3.76(\mathrm{~s}, 3 \mathrm{H}), 3.68(\mathrm{~d}, \mathrm{~J}=1.5 \mathrm{~Hz}, 1 \mathrm{H}), 3.60(\mathrm{~s}, 3 \mathrm{H}), 3.29(\mathrm{dd}, \mathrm{J}=16.0,7.5 \mathrm{~Hz}, 1 \mathrm{H})$, $3.20(\mathrm{dd}, \mathrm{J}=16.0,7.2 \mathrm{~Hz}, 1 \mathrm{H}), 1.74(\mathrm{~s}, 3 \mathrm{H}), 1.68(\mathrm{~s}, 3 \mathrm{H}) \mathrm{ppm} ;{ }^{13} \mathrm{C} \mathrm{NMR}\left(126 \mathrm{MHz}, \mathrm{CDCl}_{3}\right): \delta=166.0$, $160.3,155.7,133.3,132.8,130.1,129.5,128.0,125.8,122.4,114.4,110.5,106.4,84.5,84.5,78.9$, 59.0, 55.4, 27.8, 25.9, 17.9 ppm; IR (neat): $\tilde{v}=3277,3059,2954,2923,2869,2853,1684,1621,1603$, 1510, 1462, 1419, 1377, 1306, 1254, 1221, 1188, 1172, 1105, 1079, 1032, 989, 974, 940, 929, 903, 867, 829, 810, 767, 755, 734, 707, 698, 974, 661, $652 \mathrm{~cm}^{-1}$; HRMS (ESI): $\mathrm{m} / \mathrm{z}$ calculated for $\mathrm{C}_{22} \mathrm{H}_{25} \mathrm{NNaO}_{5}{ }^{+}\left([\mathrm{M}+\mathrm{Na}]^{+}\right)$: 406.1625; found: 406.1627. The NMR data match those reported for Peniprequinolone. ${ }^{6}$ 


\section{References}

(1) Kang, D.; Lee, J.; Lee, H.-Y. Org. Synth. 2012, 89, 66-72

(2) Wulischleger, C. W.; Gertsch, J.; Altmann, K. H. Org. Lett. 2010, 12, 1120-1123.

(3) Mukaiyama, T.; Shiina, I.; Iwadare, H.; Saitoh, M.; Nishimura, T.; Ohkawa, N.; Sakoh, H.; Nishimura, K.; Tani, Y.; Hasegawa, M.; Yamada, K.; Saitoh, K. Chem. Eur. J. 1999, 5, 121-161.

(4) Suguru, Y.; Ken, S.; Takako, N.; Takamitsu, H. Chem. Lett. 2015, 44, 1324-1326.

(5) An, C.-Y.; Li, X.-M.; Luo, H.; Li, C.-S.; Wang, M.-H.; Xu, G.-M.; Wang, B.-G., J. Nat. Prod. 2013, 76, 1896-1901.

(6) Hayashi, H.; Nakatani, T.; Inoue, Y; Nakayama, M.; Nozake, H.; Biosci. Biotech. Biochem. 1997, 61, 914-916;

(7) Neff, S. A.; Lee, S. U.; Asami, Y.; Ahn, J. S.; Oh, H.; Baltrusaitis, J.; Gloer, J. B.; Wicklow, D. T.; J. Nat. Prod. 2012, 75, 464-472. 


\section{Appendix}

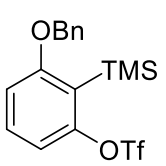

$3 b$

${ }^{1} \mathrm{H}$ NMR $\left(500 \mathrm{MHz}, \mathrm{CDCl}_{3}\right)$
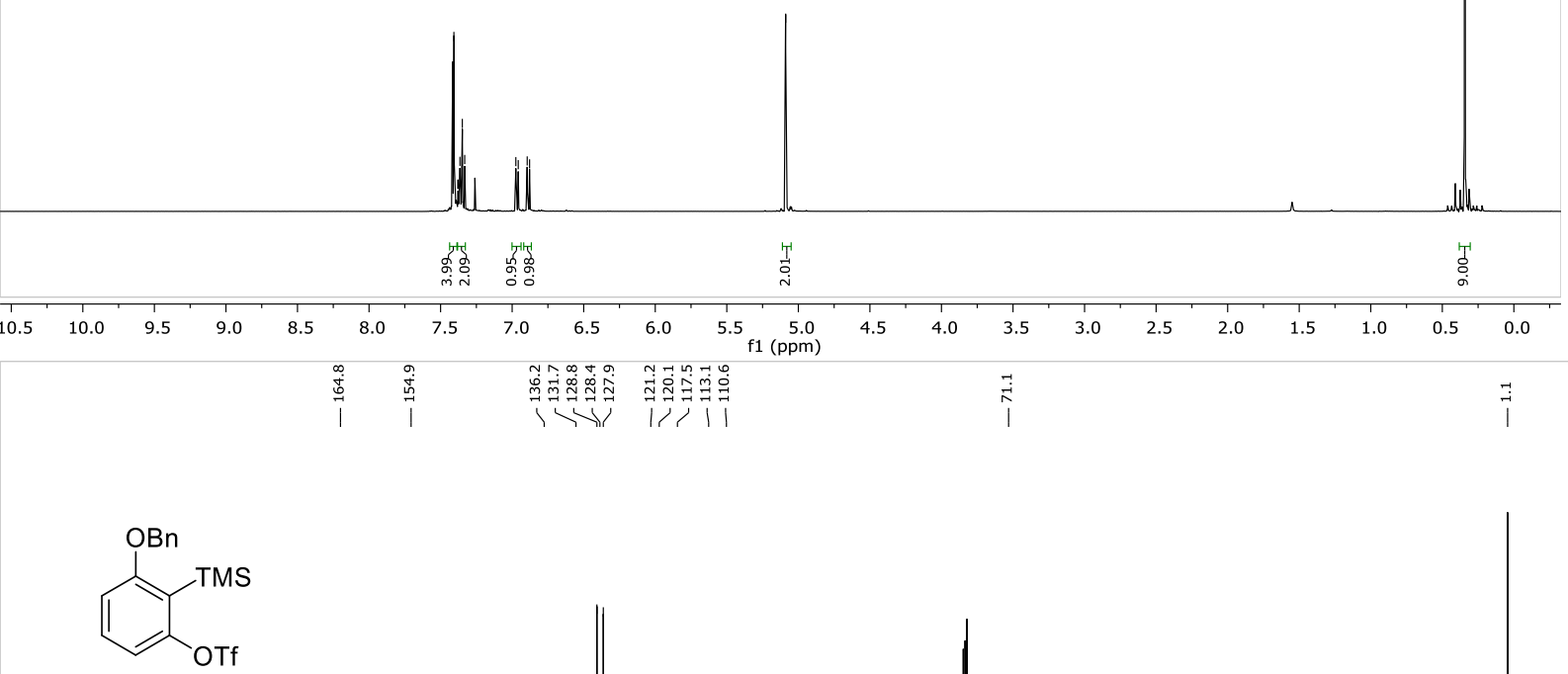

3b

${ }^{13} \mathrm{C} \mathrm{NMR}\left(126 \mathrm{MHz}, \mathrm{CDCl}_{3}\right)$

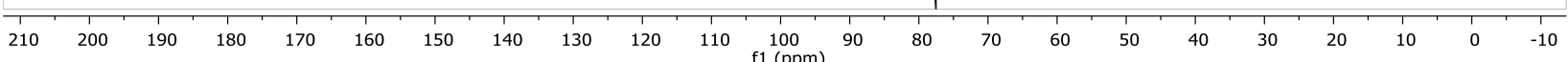




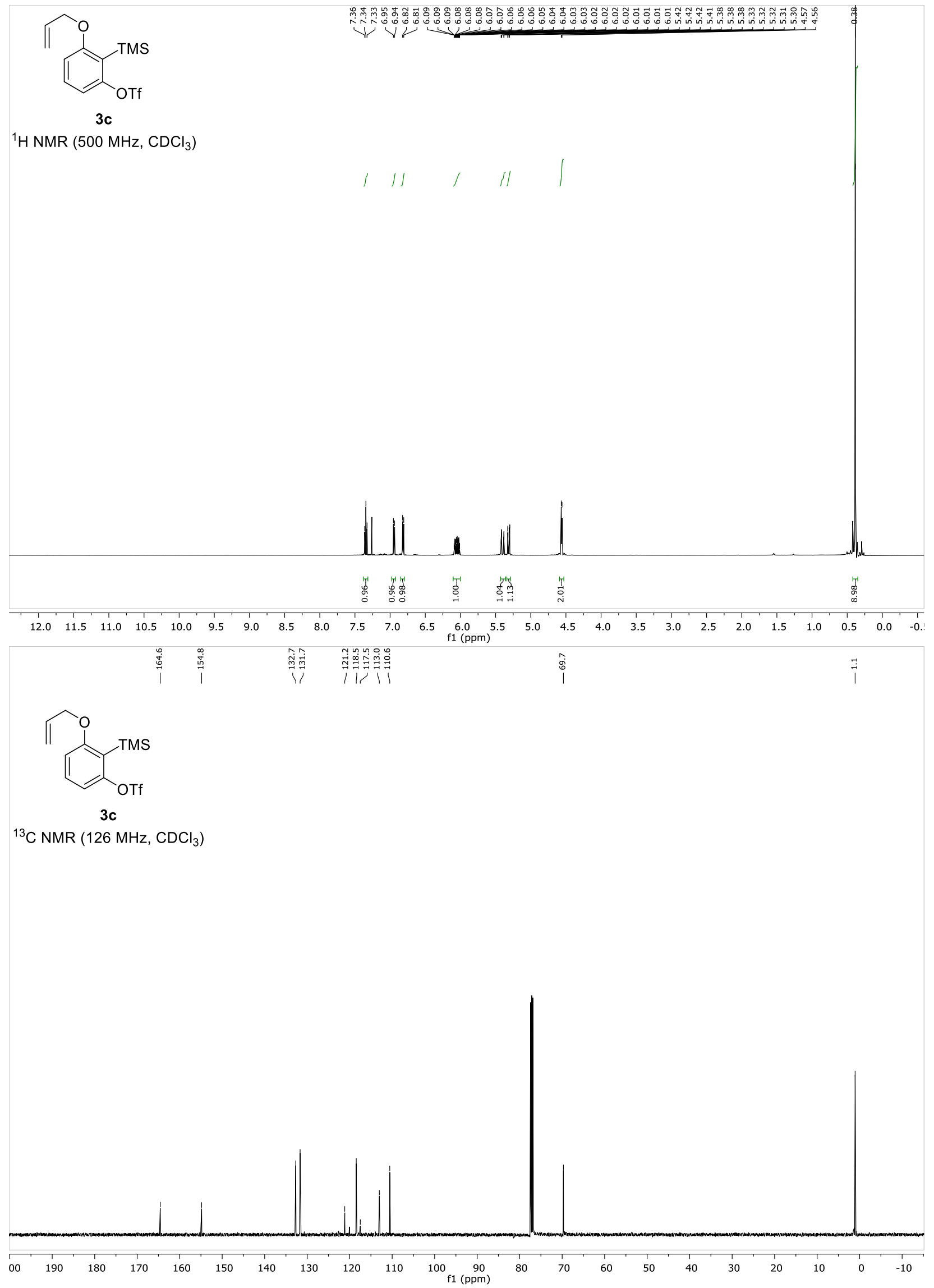



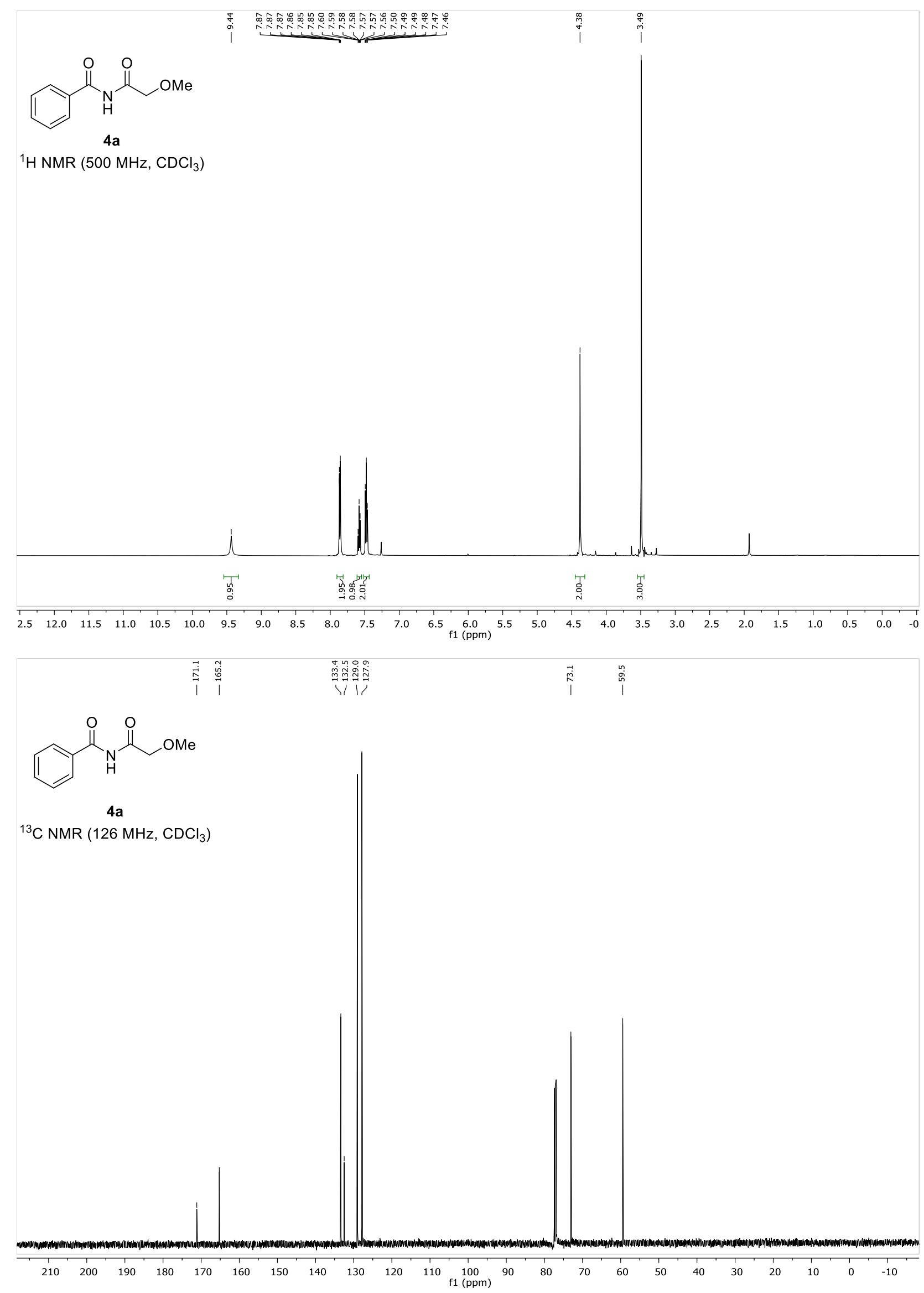

S26 

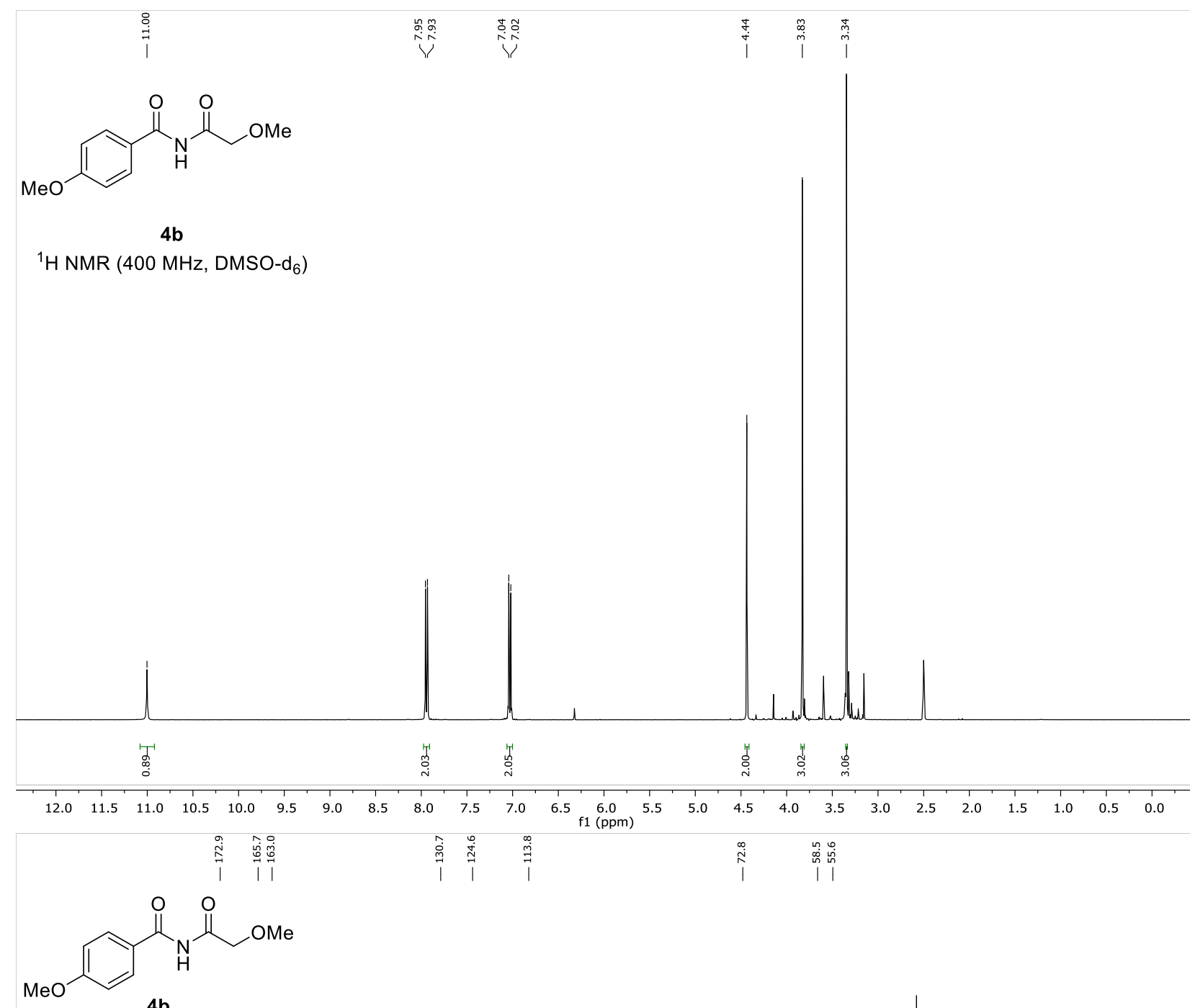

${ }^{13} \mathrm{C}$ NMR $\left(101 \mathrm{MHz}\right.$, DMSO- $\left.\mathrm{d}_{6}\right)$

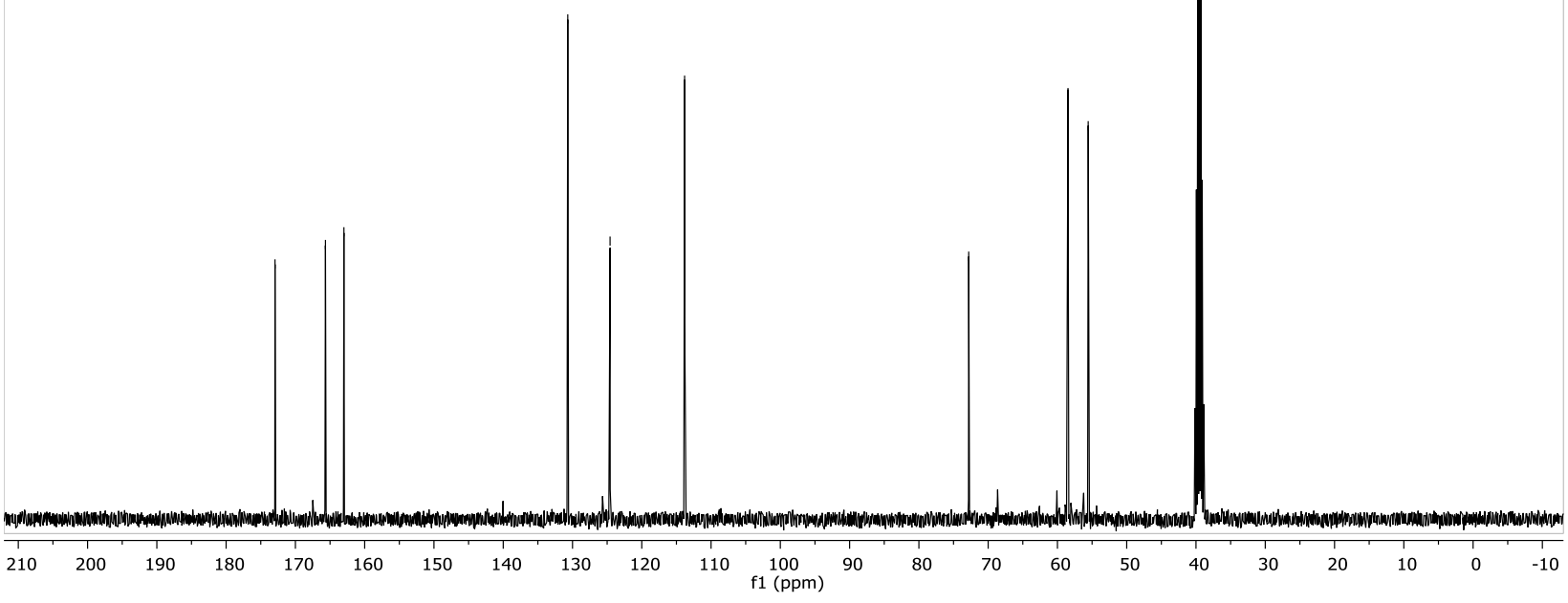




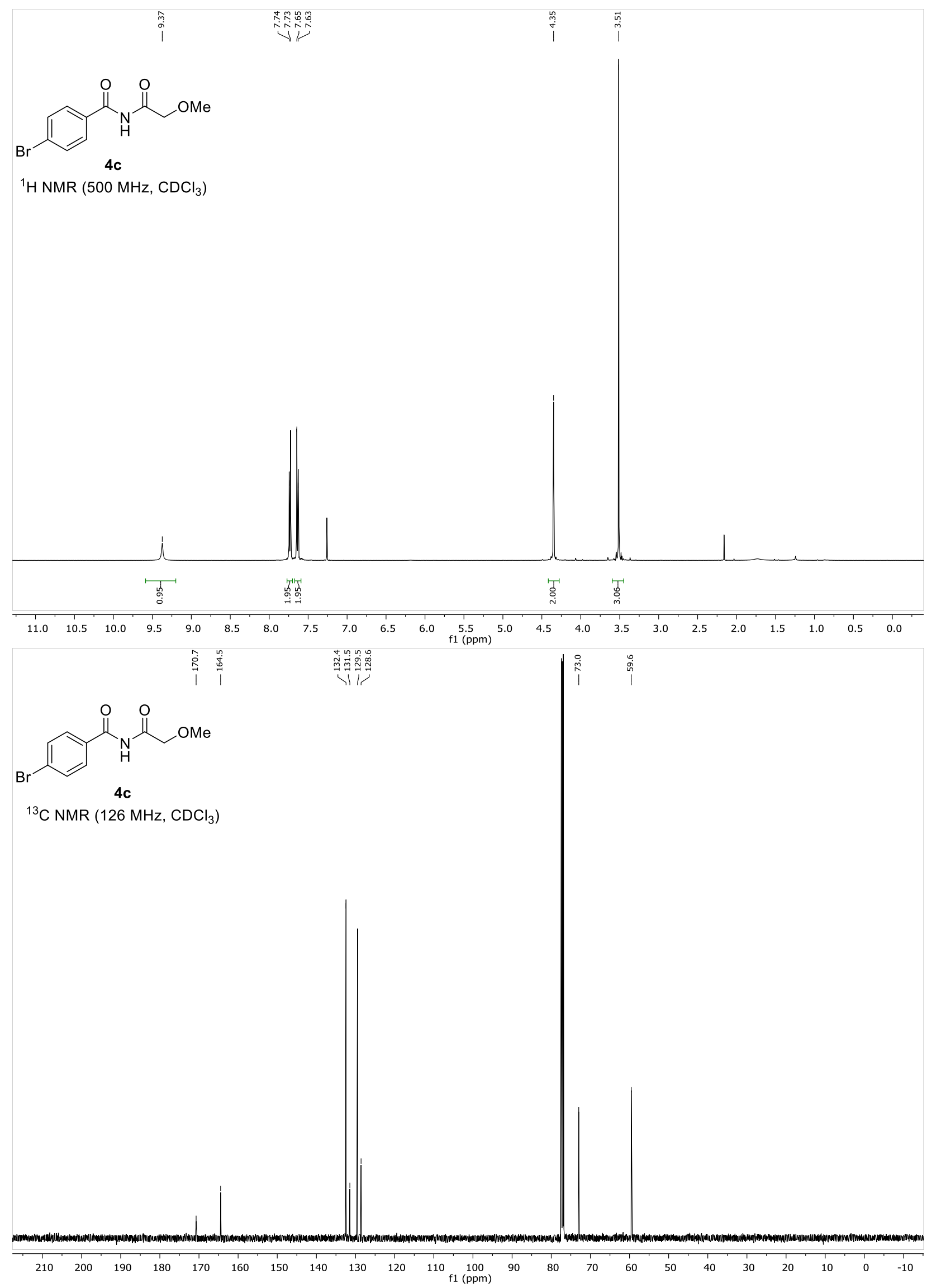




\section{|}

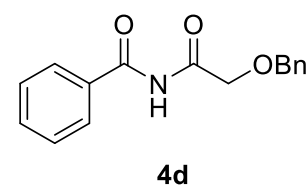

${ }^{1} \mathrm{H}$ NMR $\left(700 \mathrm{MHz}, \mathrm{CDCl}_{3}\right)$

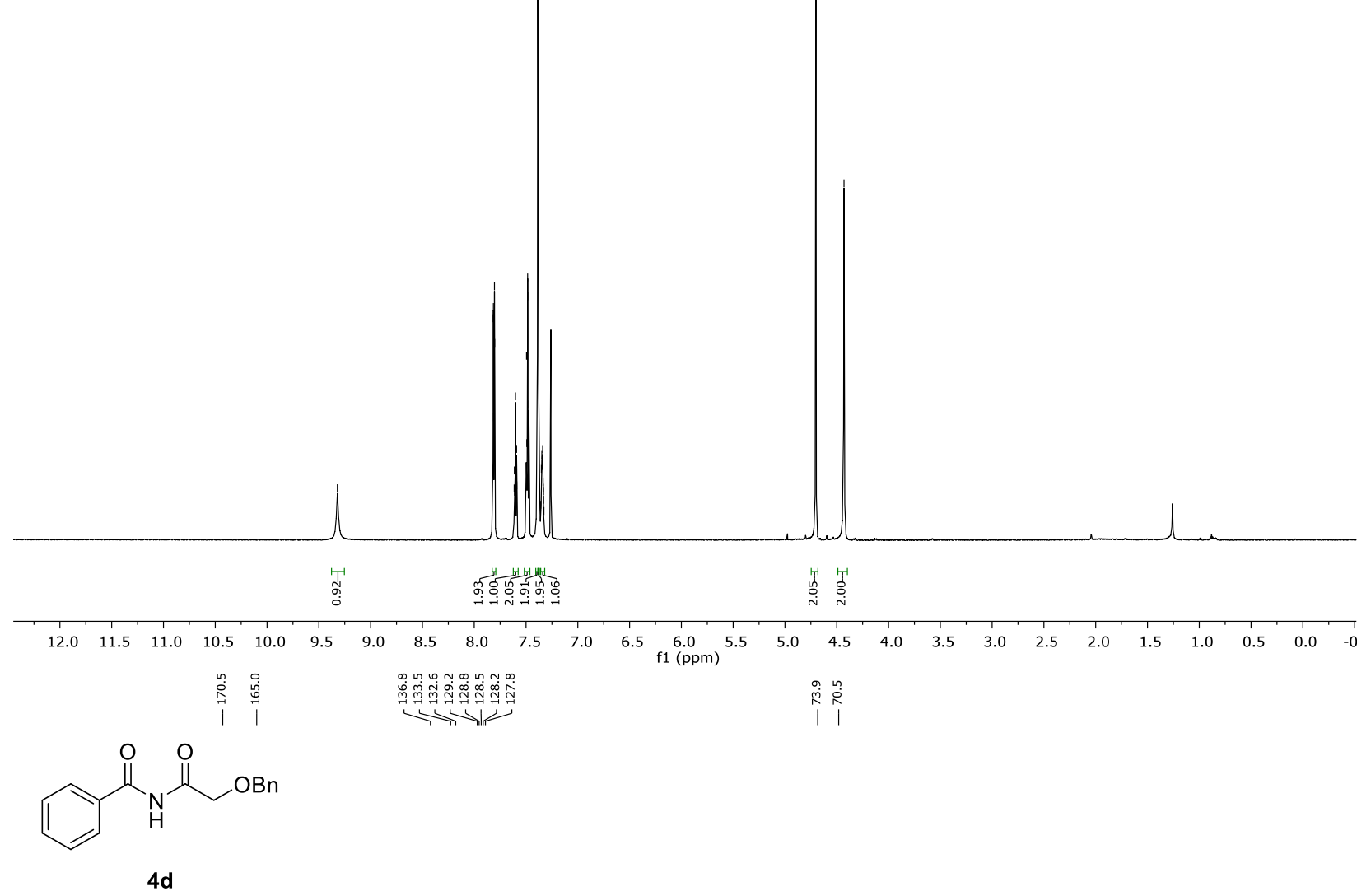

${ }^{13} \mathrm{C}$ NMR $\left(176 \mathrm{MHz}, \mathrm{CDCl}_{3}\right)$

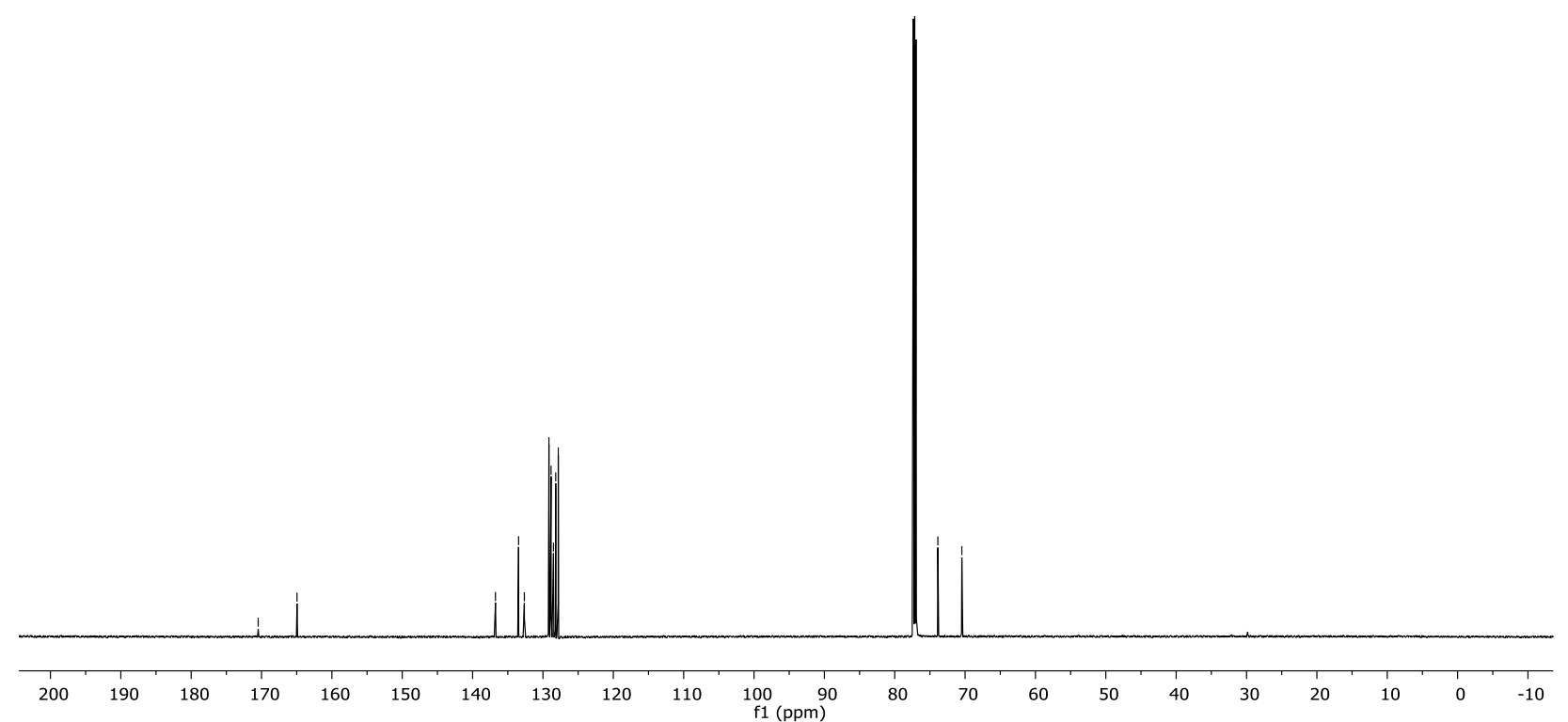




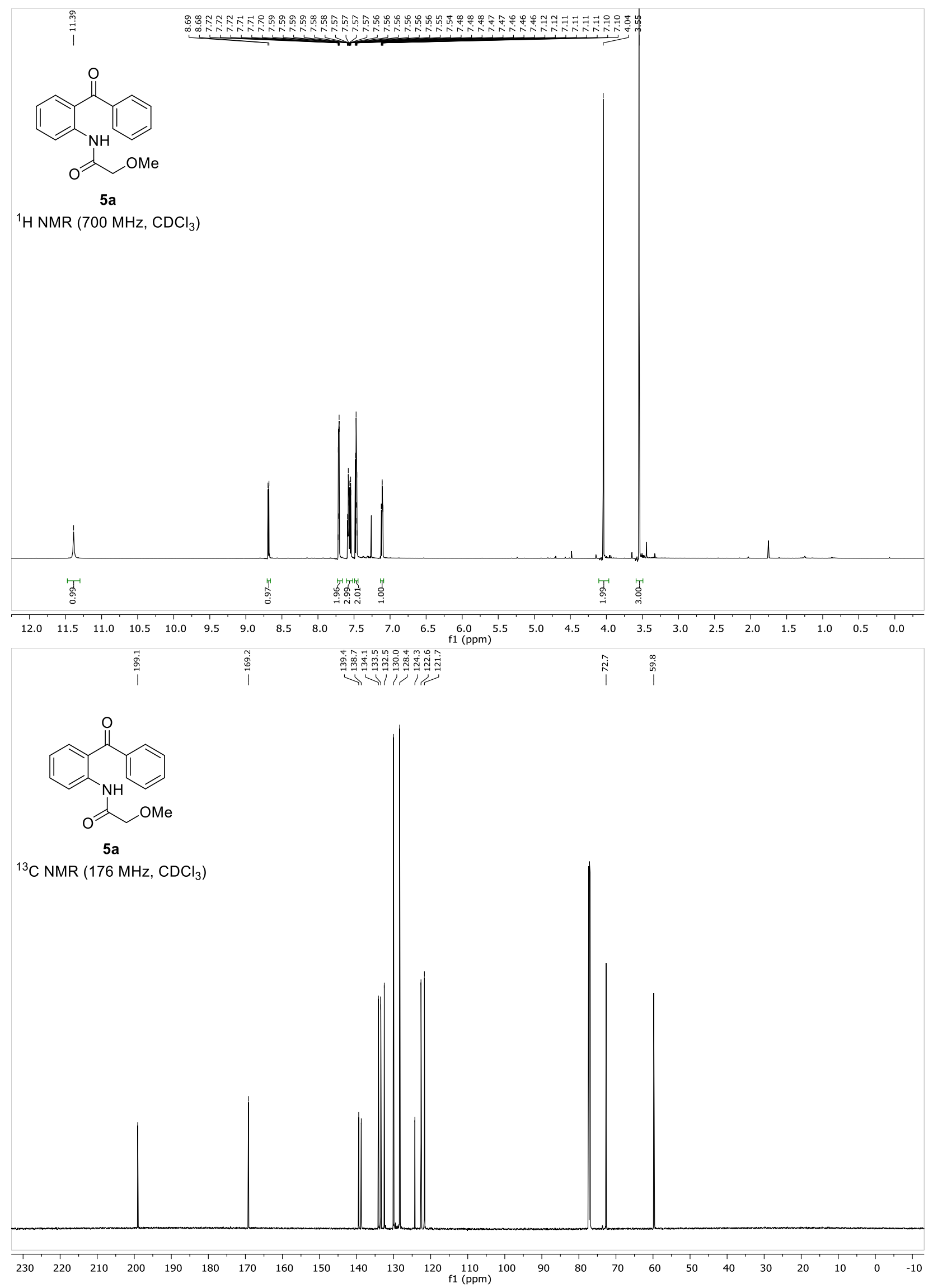




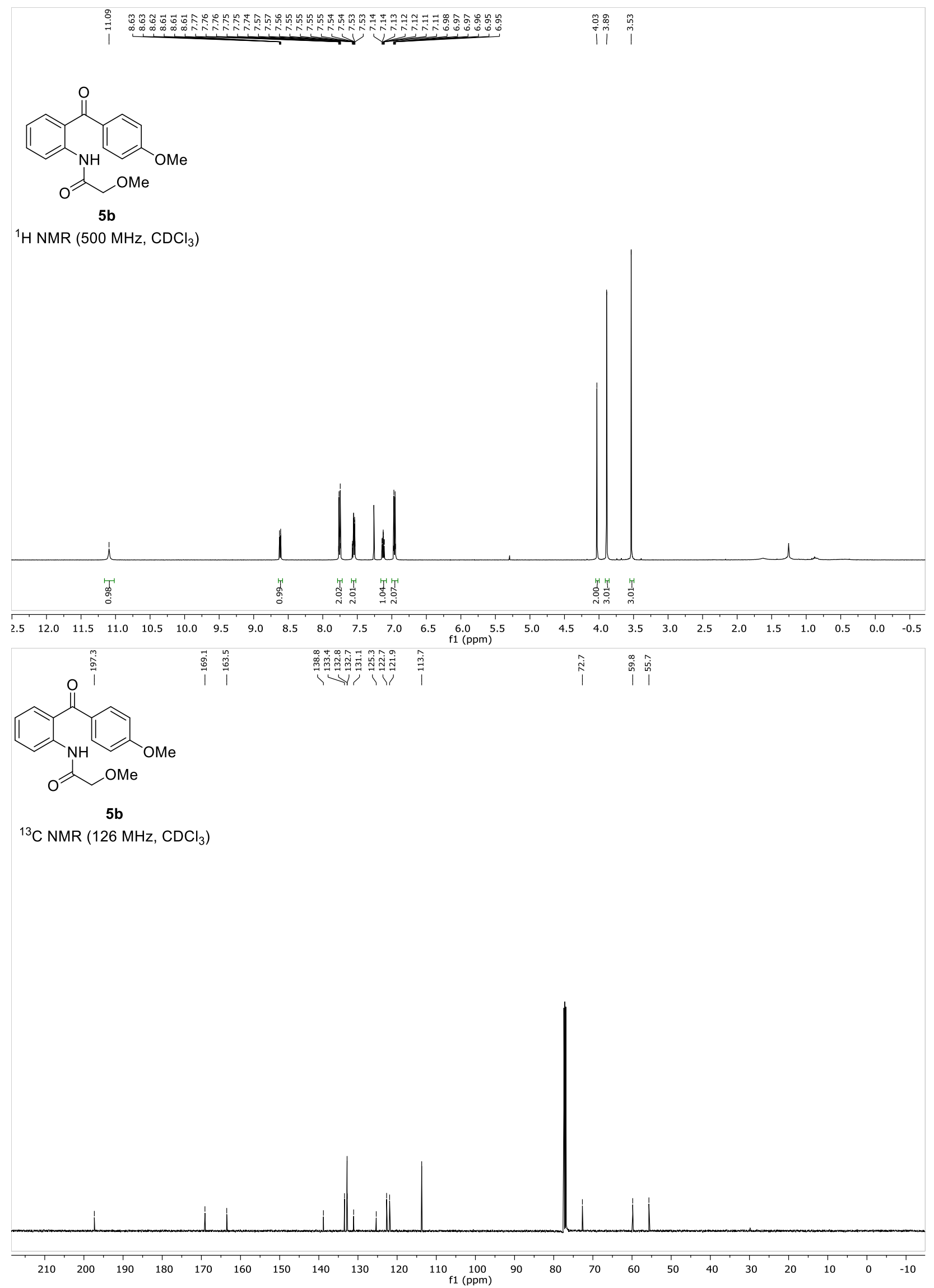




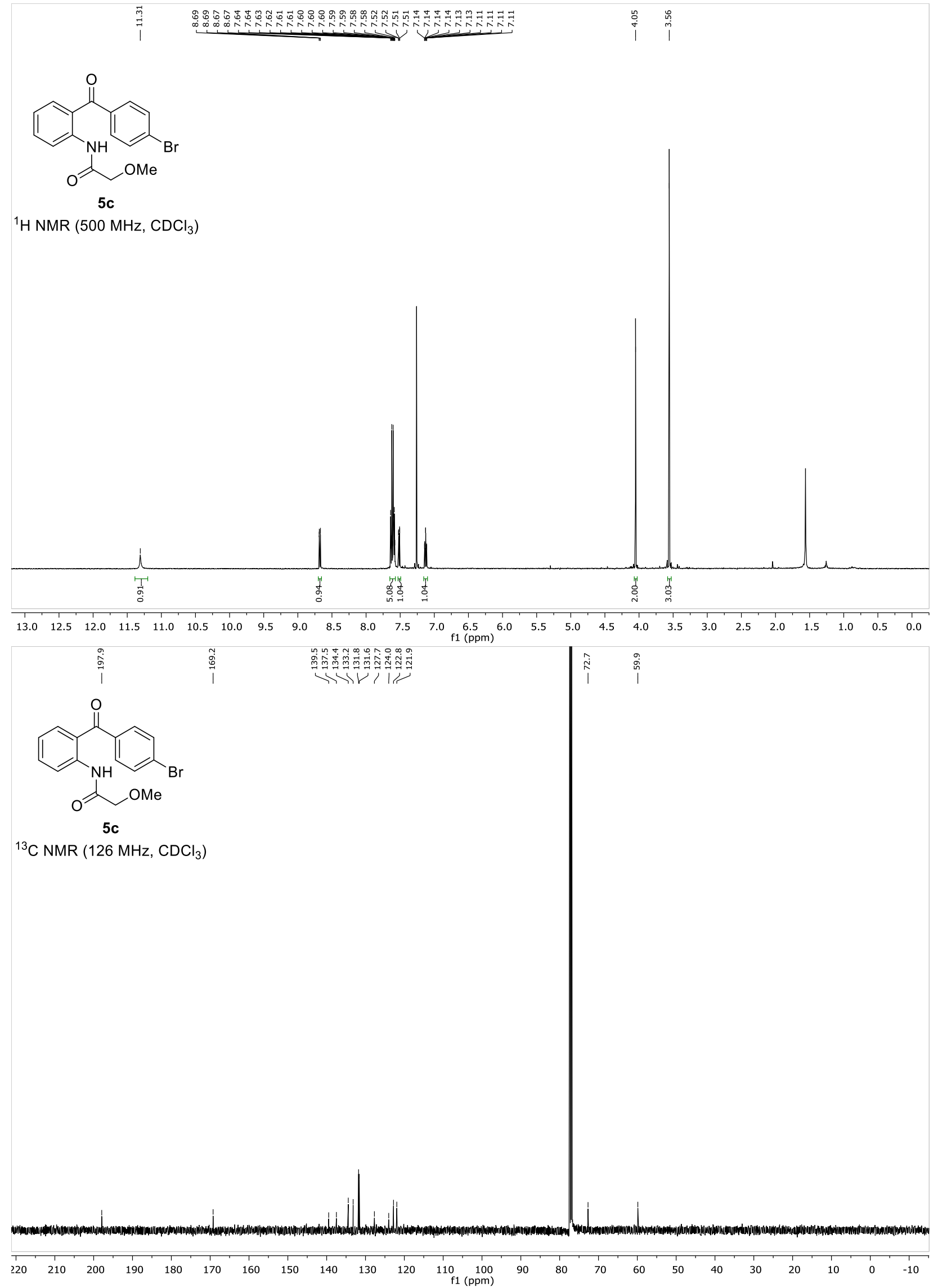




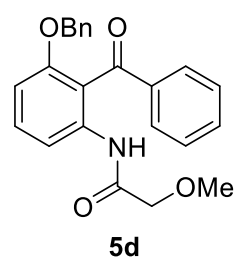

${ }^{1} \mathrm{H}$ NMR $\left(700 \mathrm{MHz}, \mathrm{CDCl}_{3}\right)$
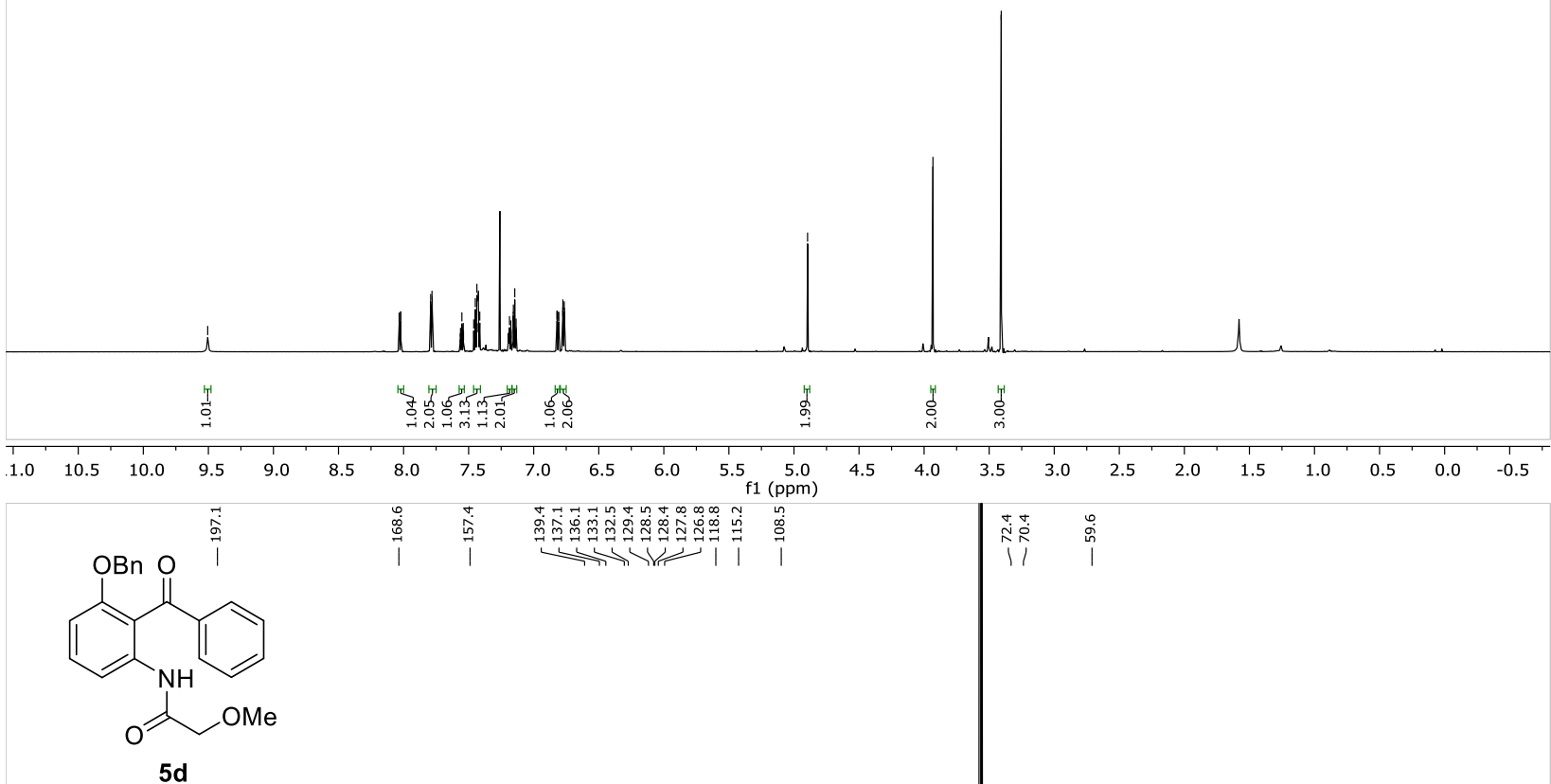

$\left.{ }^{13} \mathrm{C} \mathrm{NMR} \mathrm{(176} \mathrm{MHz,} \mathrm{CDCl}_{3}\right)$

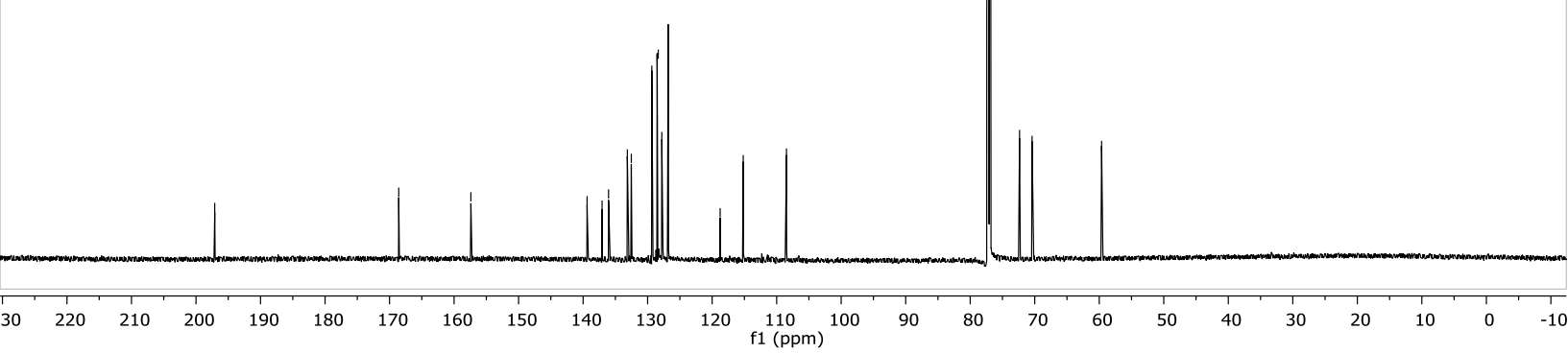




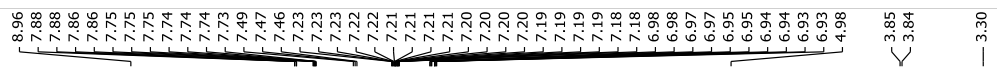

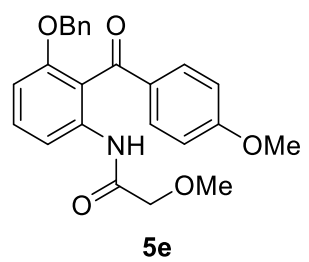

${ }^{1} \mathrm{H}$ NMR $\left(700 \mathrm{MHz}, \mathrm{CD}_{3} \mathrm{CN}\right)$
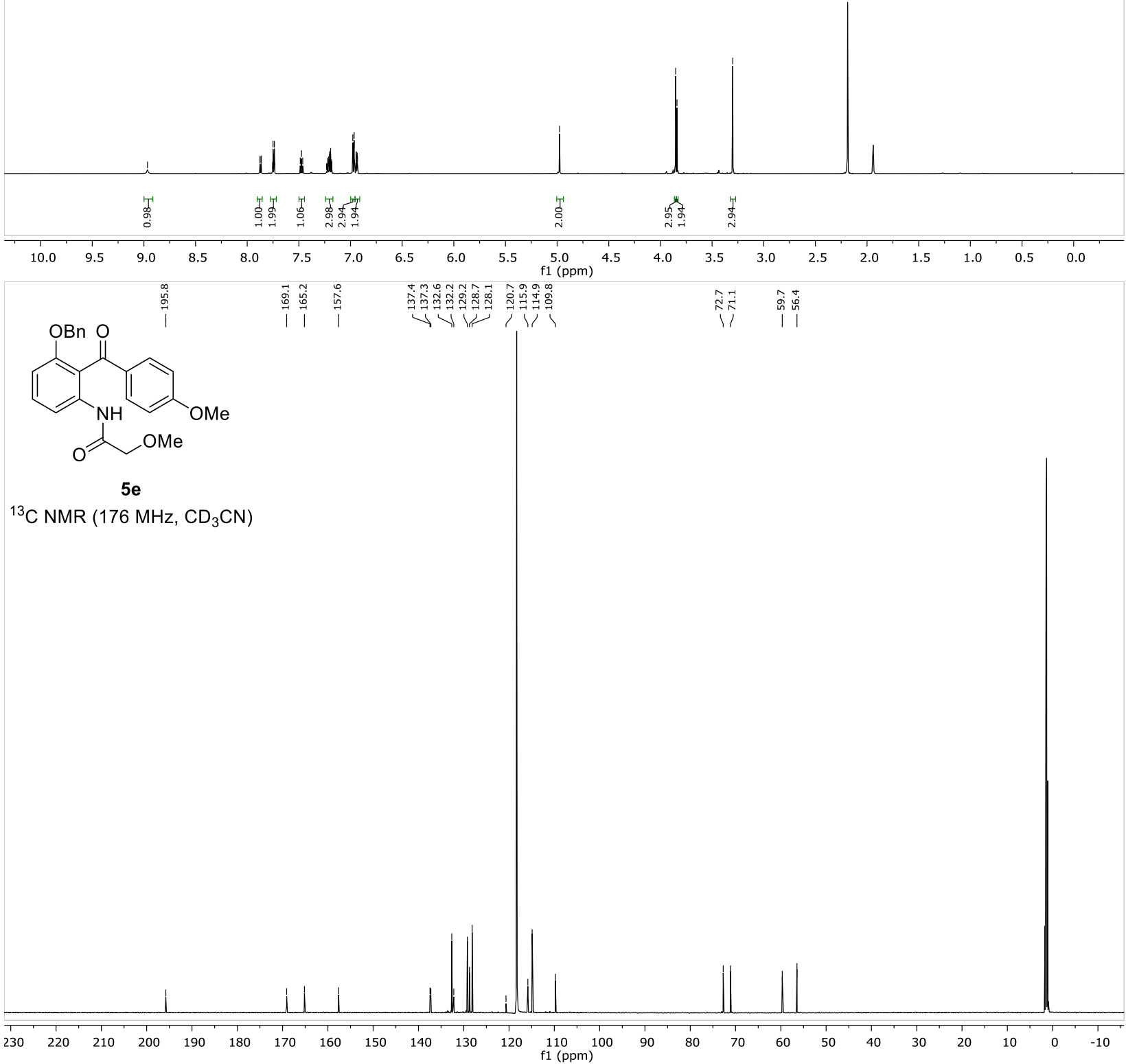


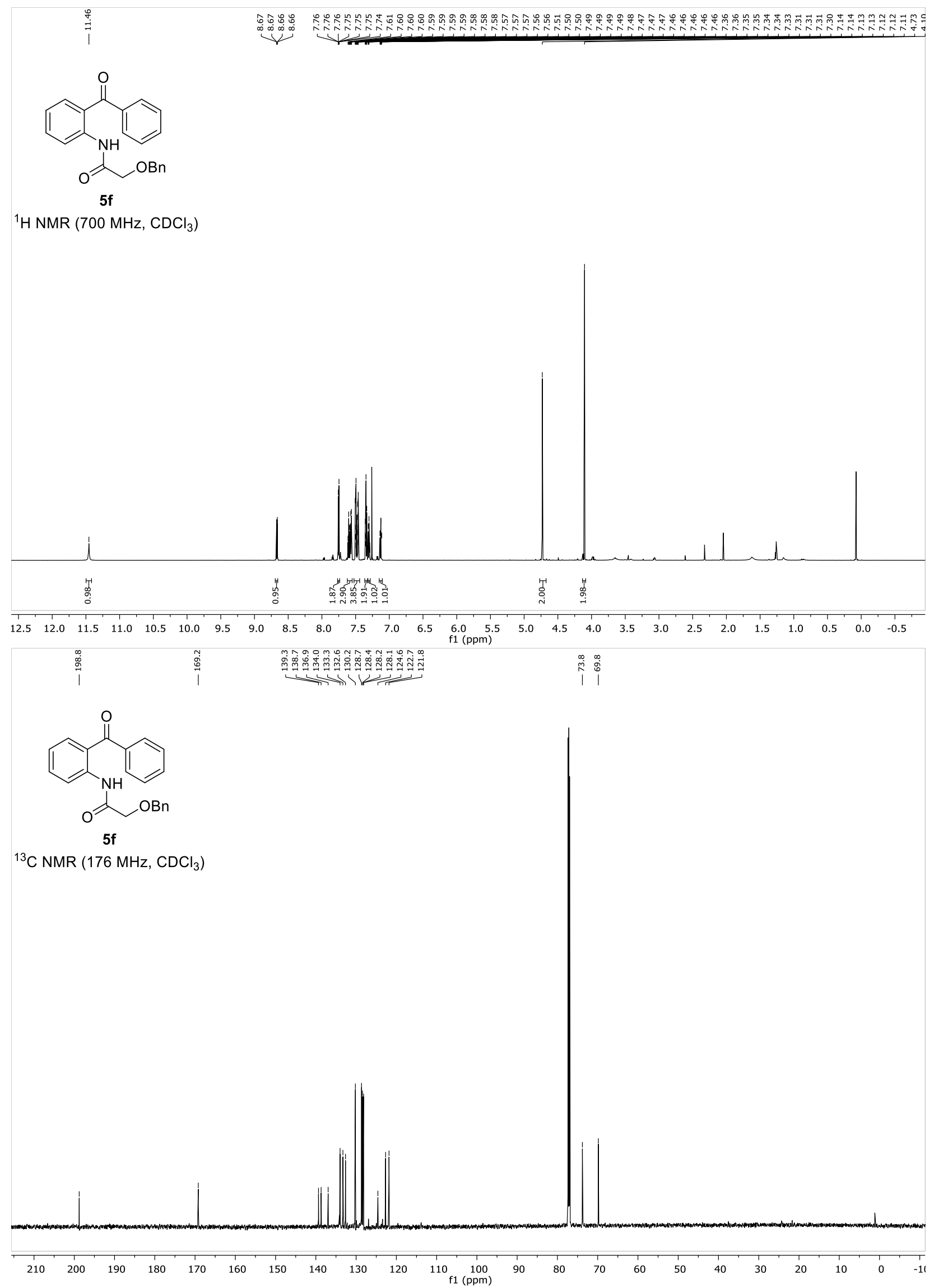




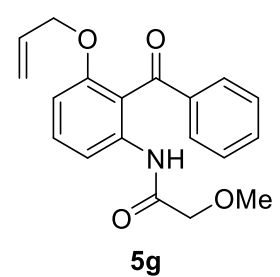

${ }^{1} \mathrm{H}$ NMR $\left(500 \mathrm{MHz}, \mathrm{CDCl}_{3}\right)$

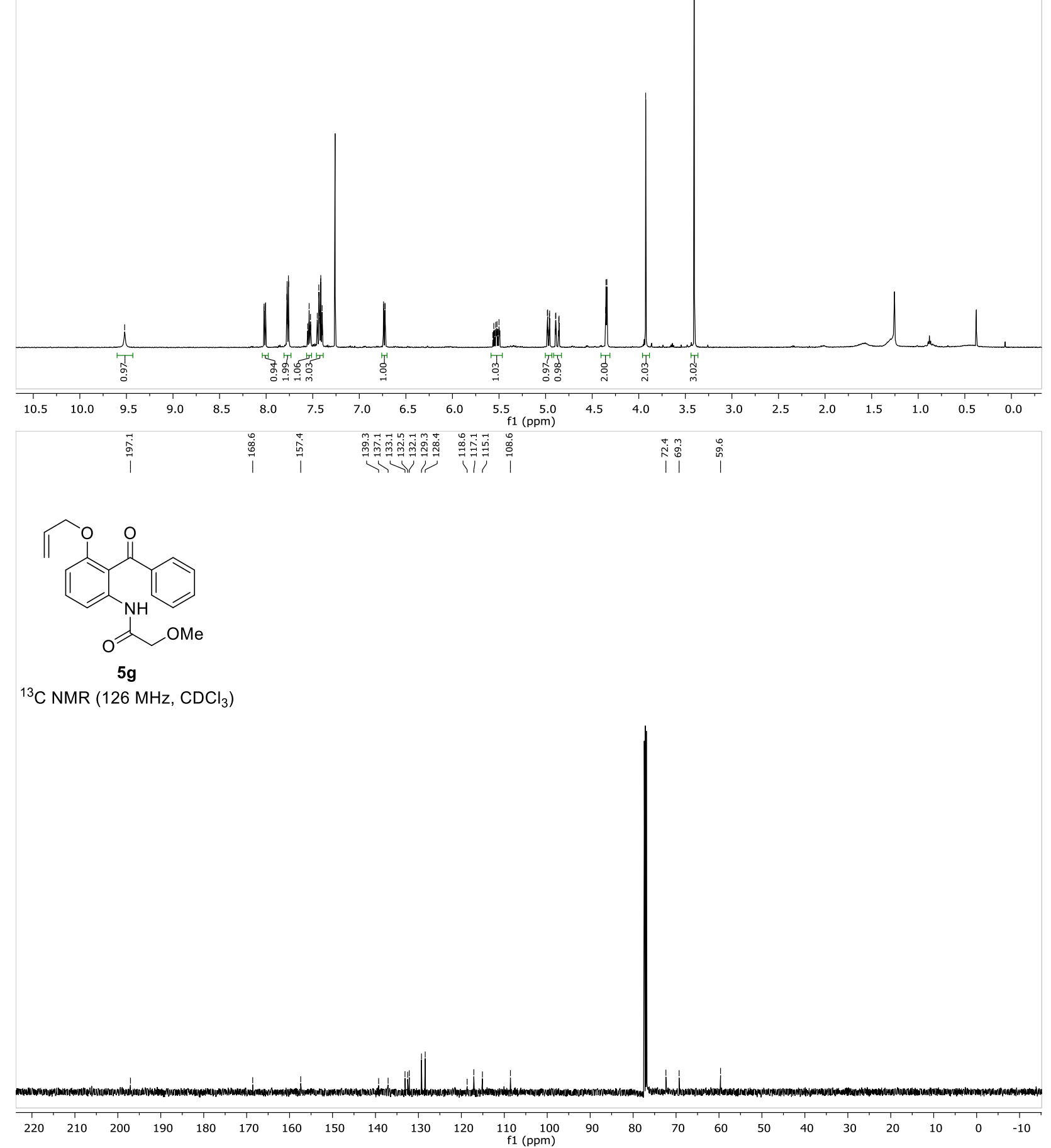




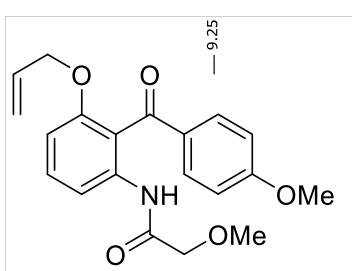

$5 \mathrm{~h}$

${ }^{1} \mathrm{H}$ NMR $\left(500 \mathrm{MHz}, \mathrm{CDCl}_{3}\right)$

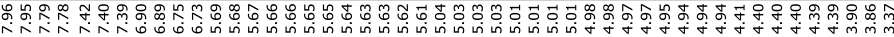
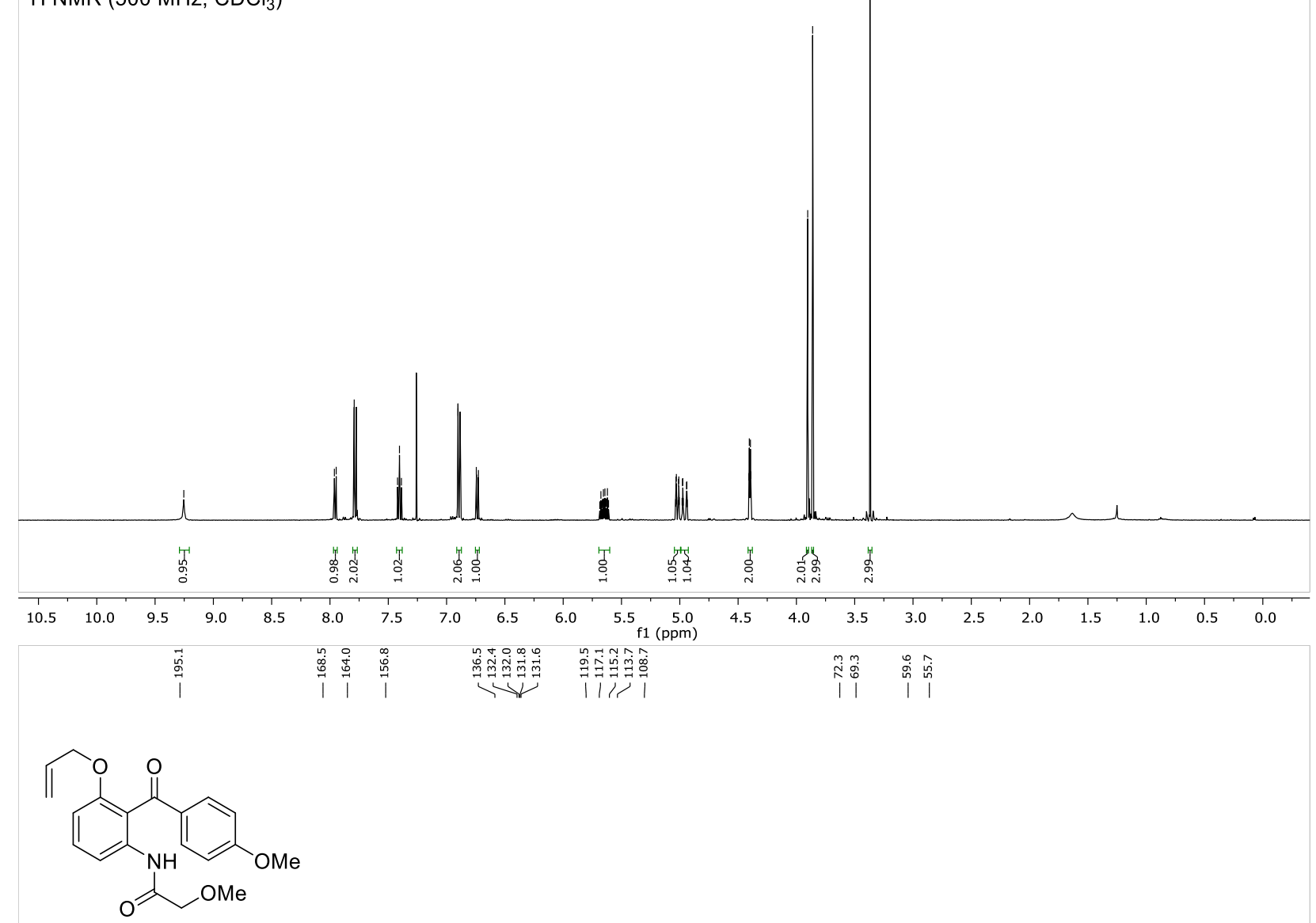

$5 \mathrm{~h}$

${ }^{13} \mathrm{C}$ NMR $\left(126 \mathrm{MHz}, \mathrm{CDCl}_{3}\right)$

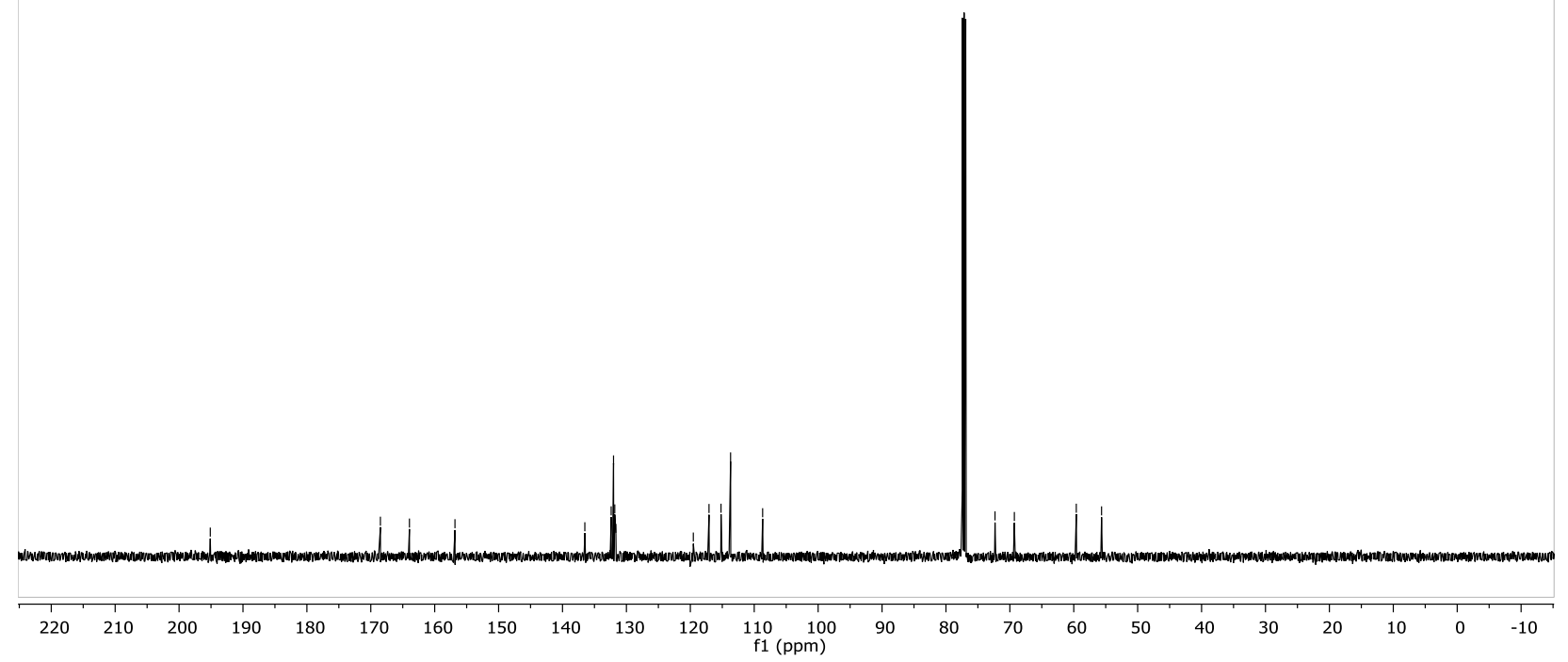




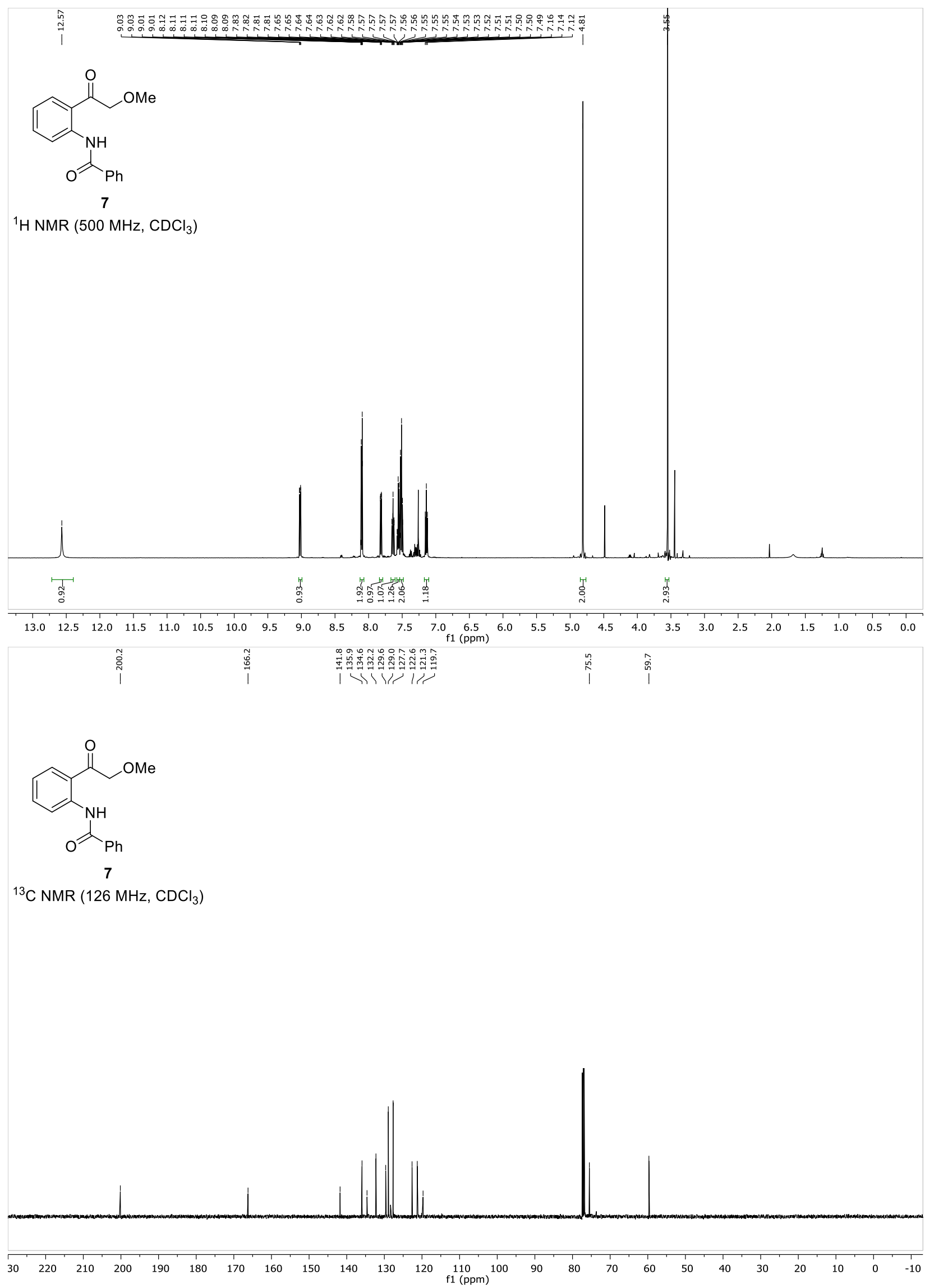

S38 


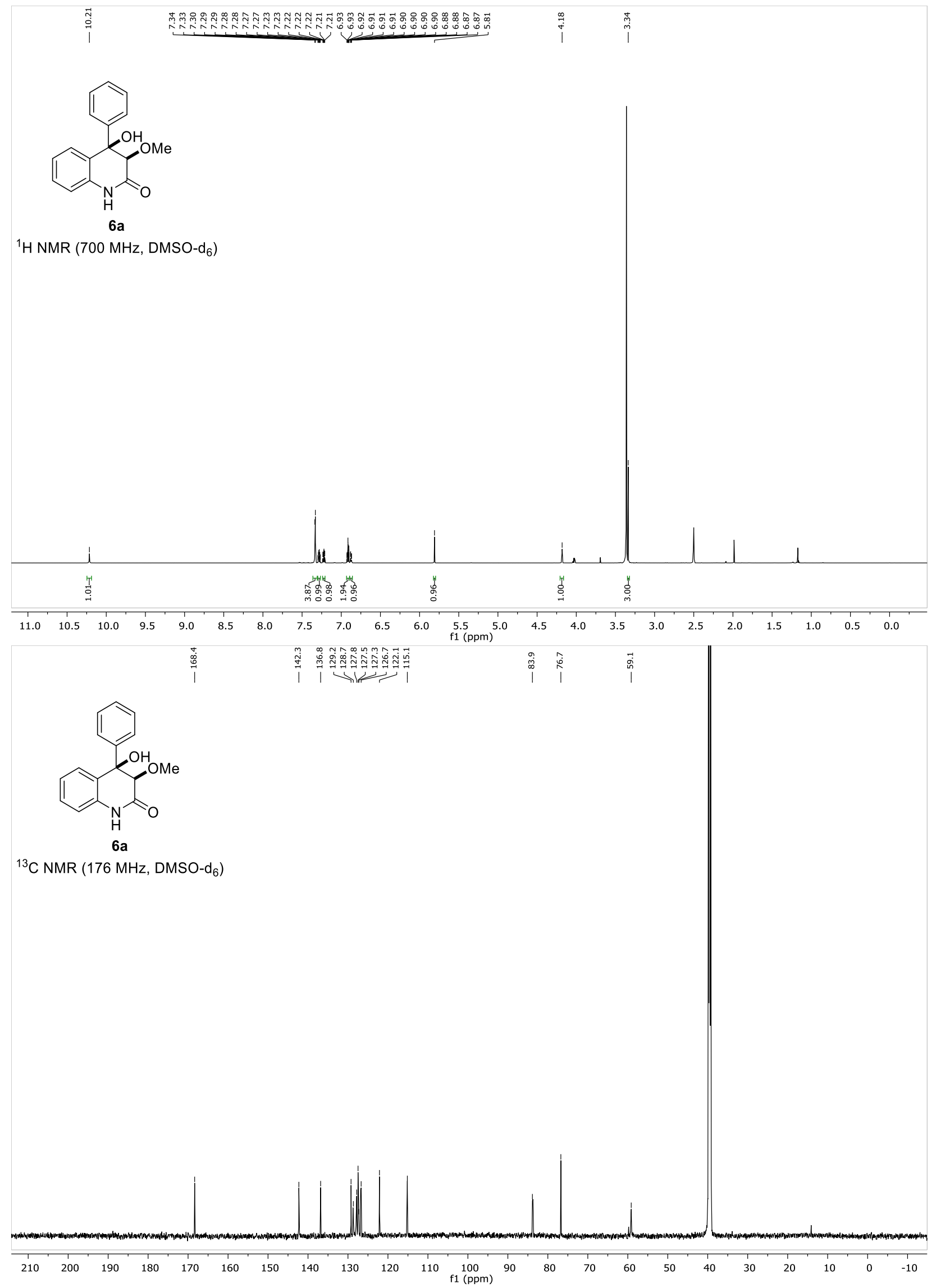




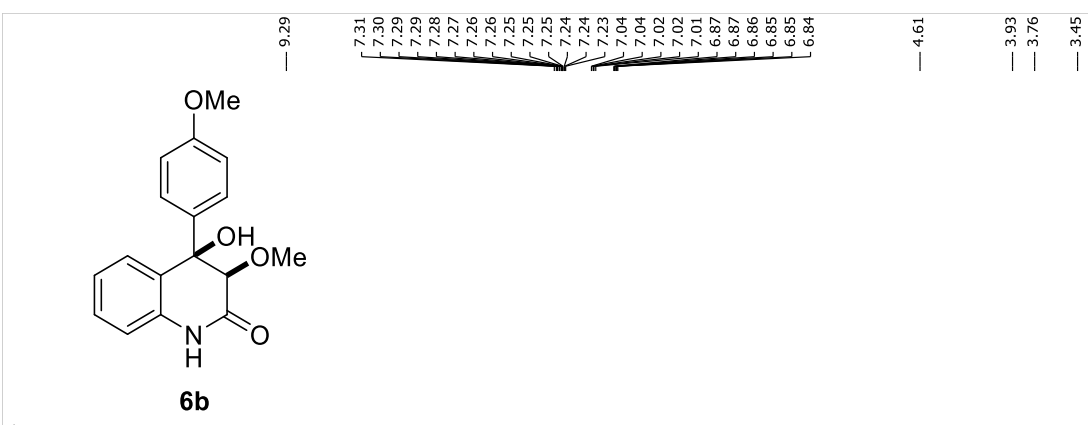

${ }^{1} \mathrm{H}$ NMR (500 MHz, acetone- $\left.d_{6}\right)$
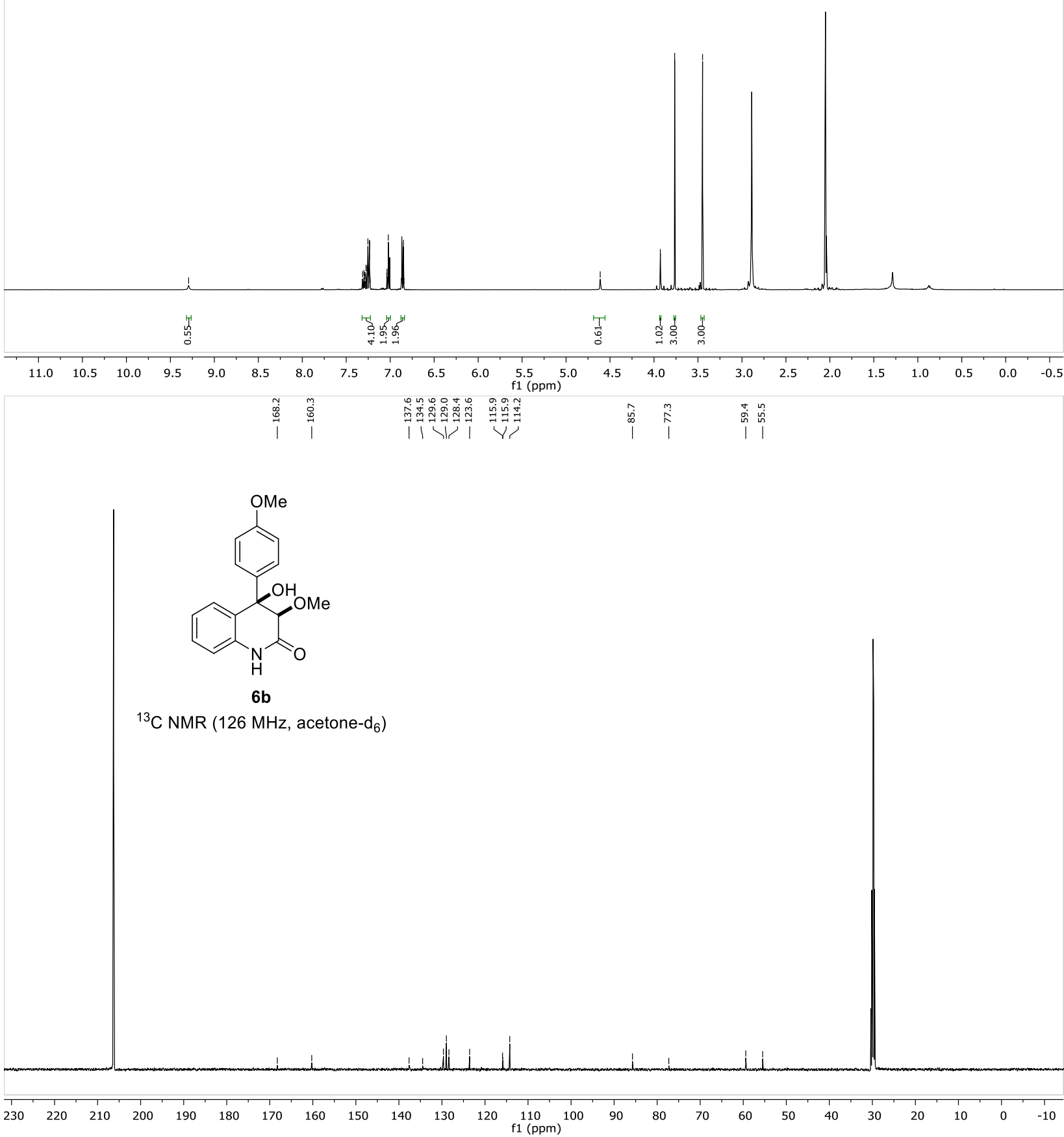


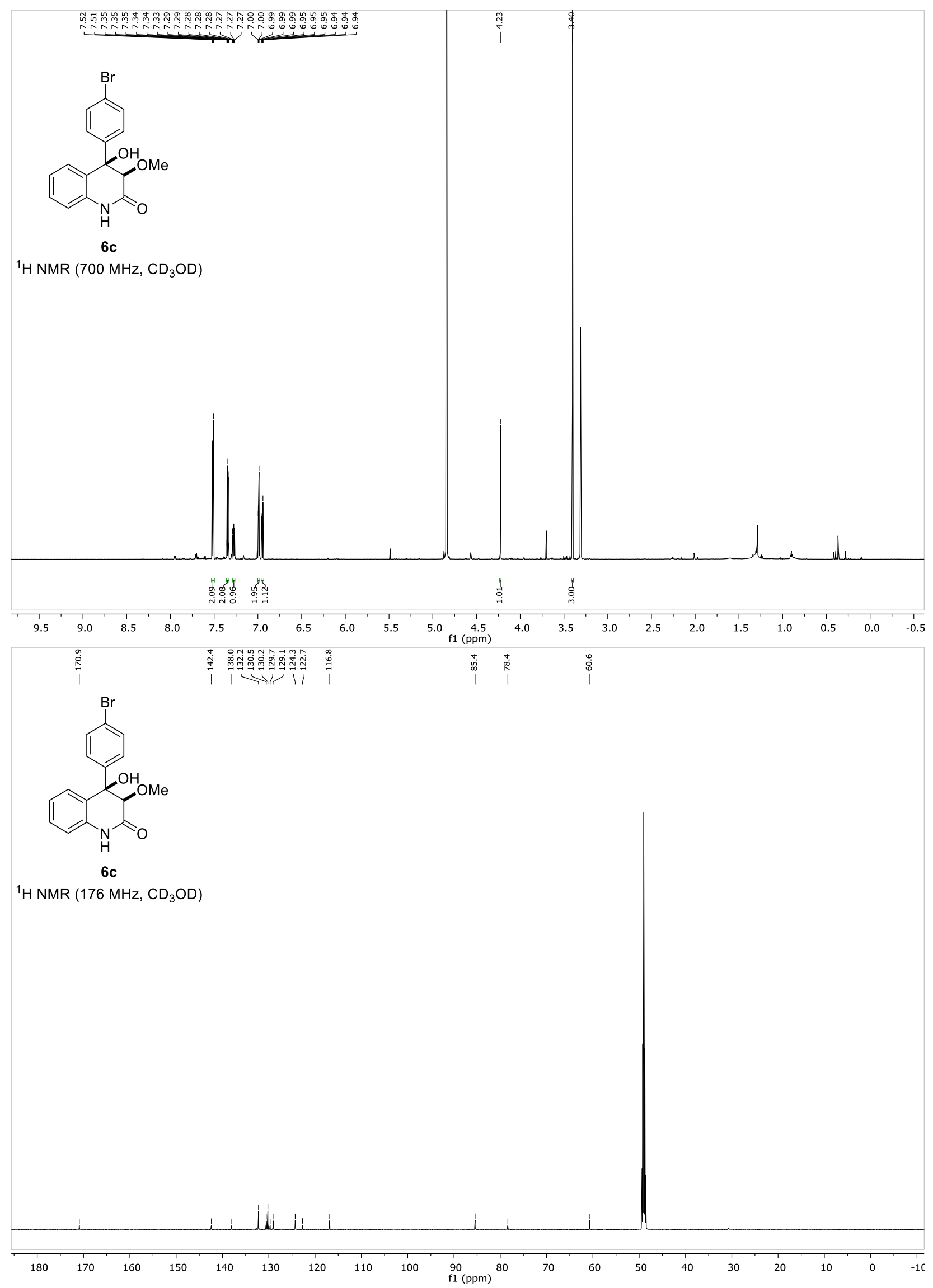




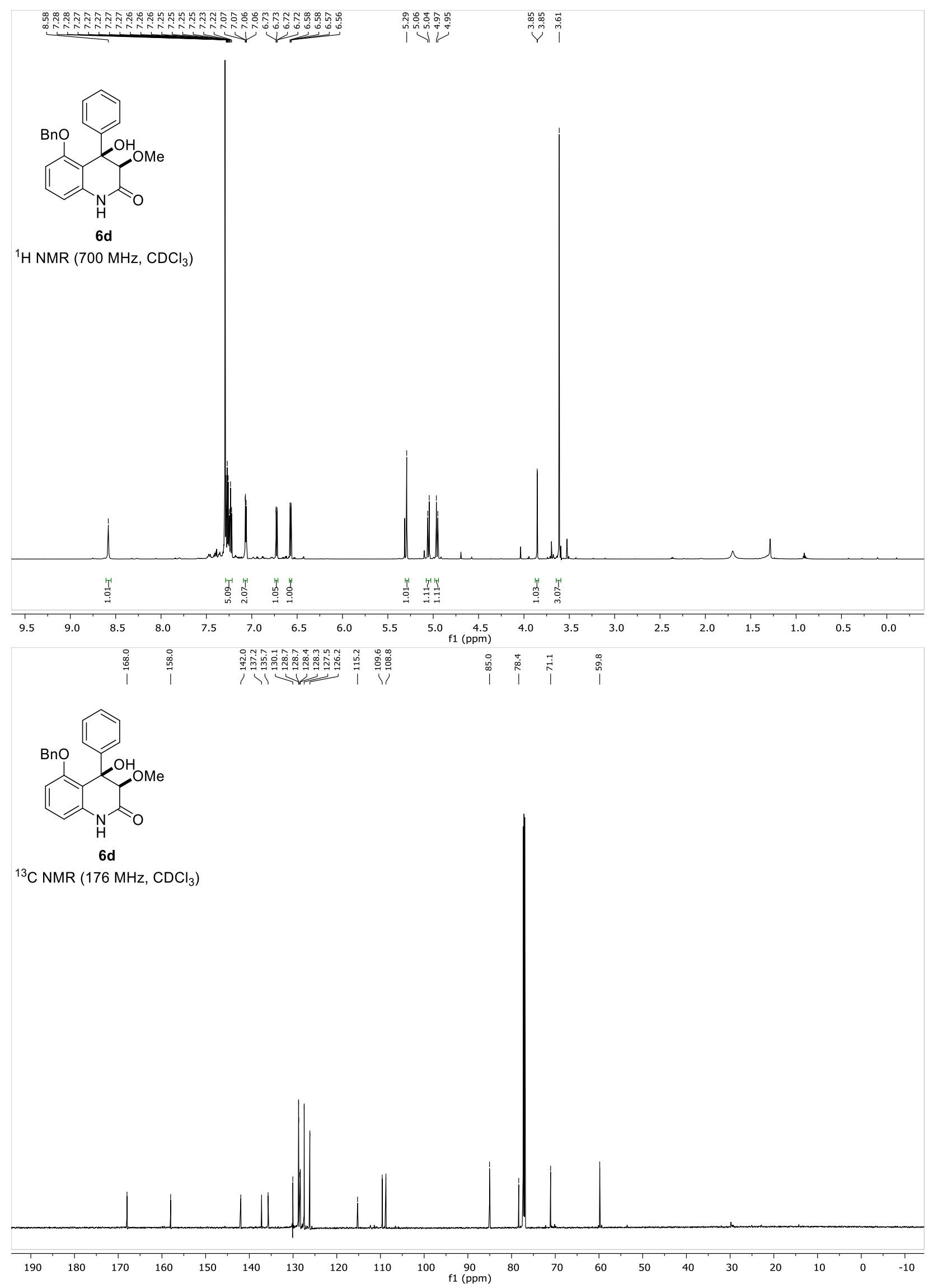




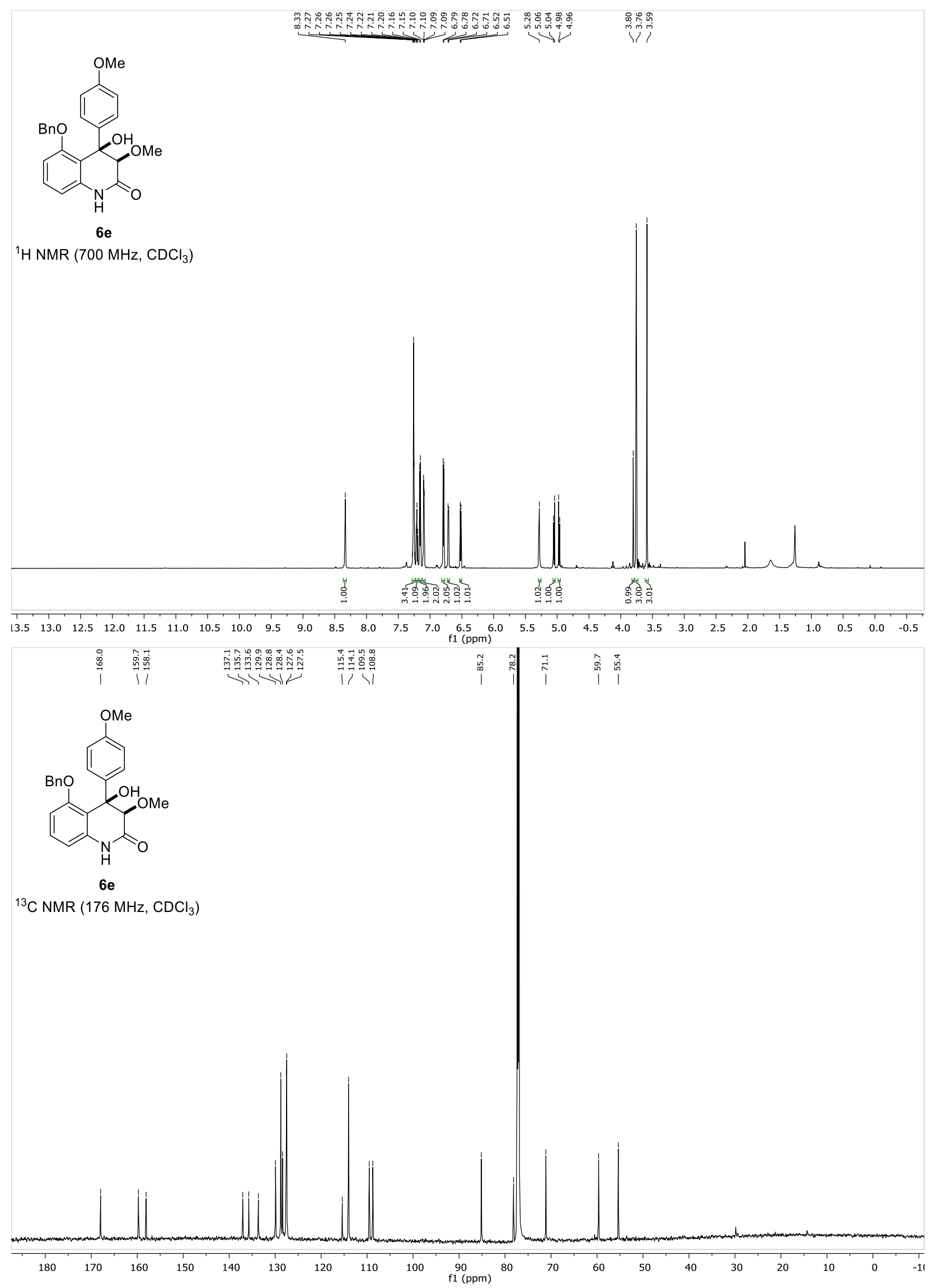




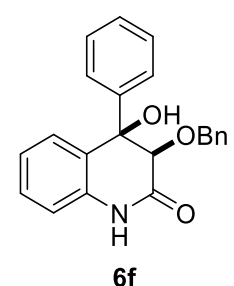

${ }^{1} \mathrm{H}$ NMR $\left(500 \mathrm{MHz}, \mathrm{CD}_{3} \mathrm{OD}\right)$

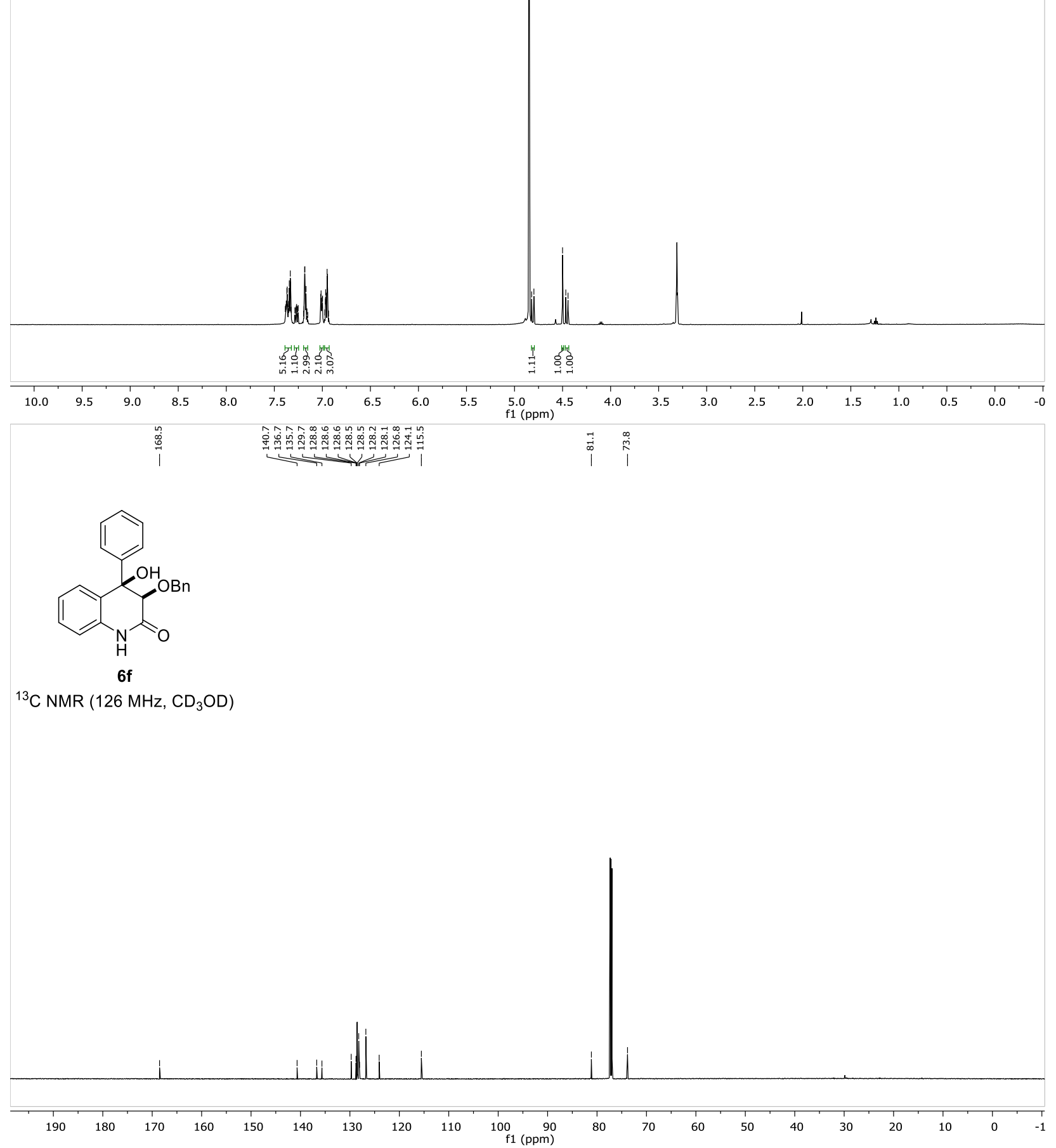




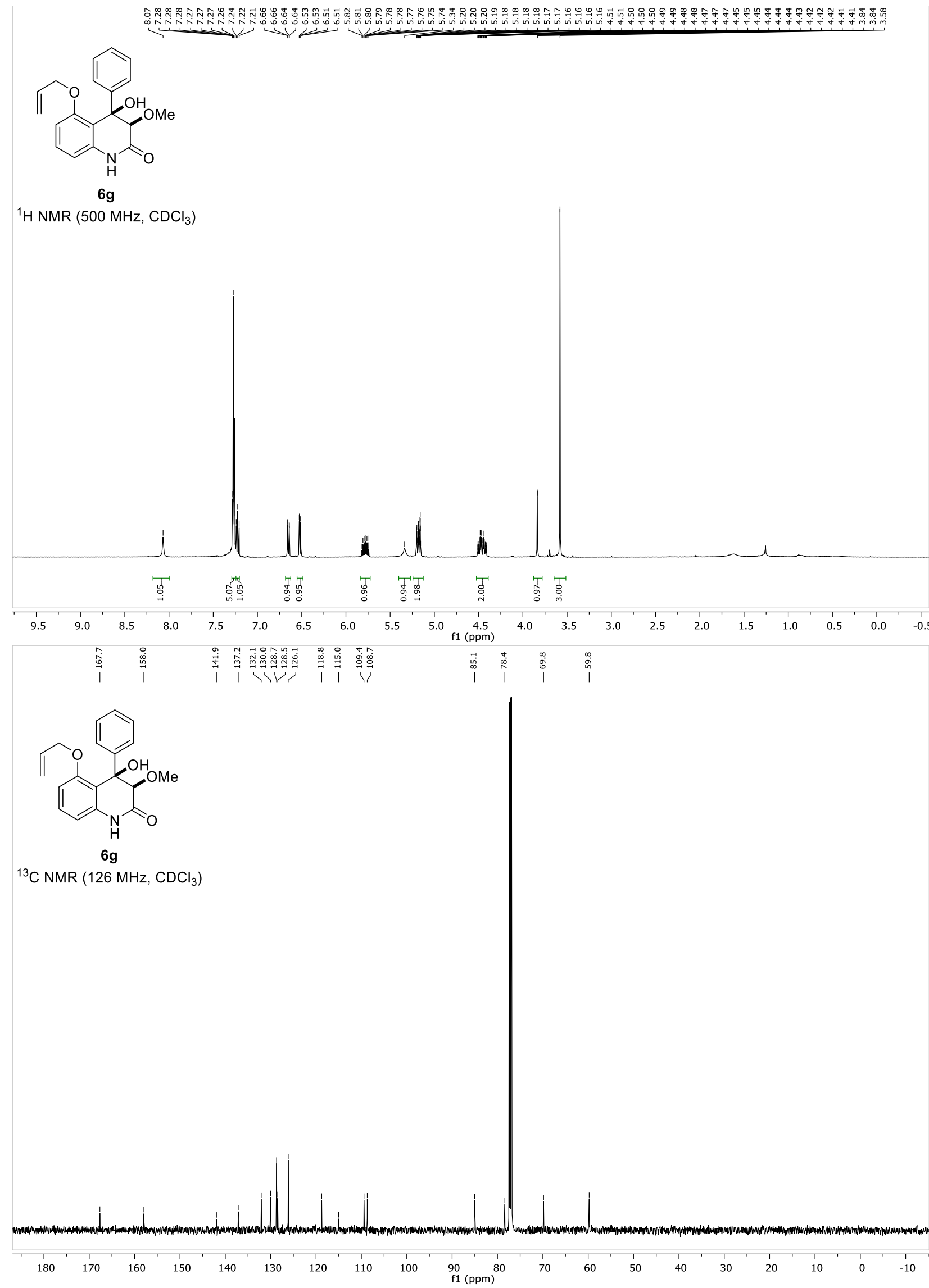




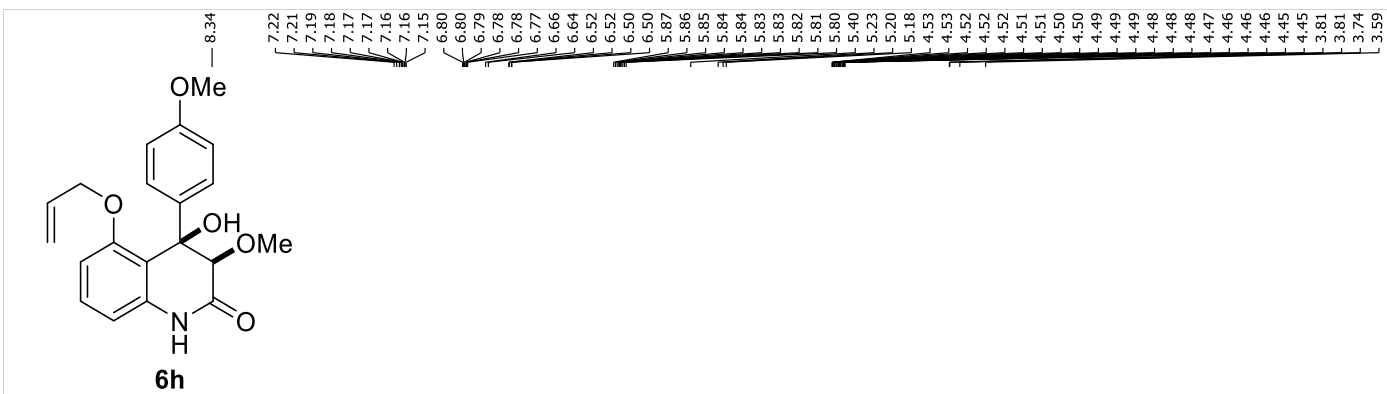

${ }^{1} \mathrm{H}$ NMR $\left(500 \mathrm{MHz}, \mathrm{CDCl}_{3}\right)$

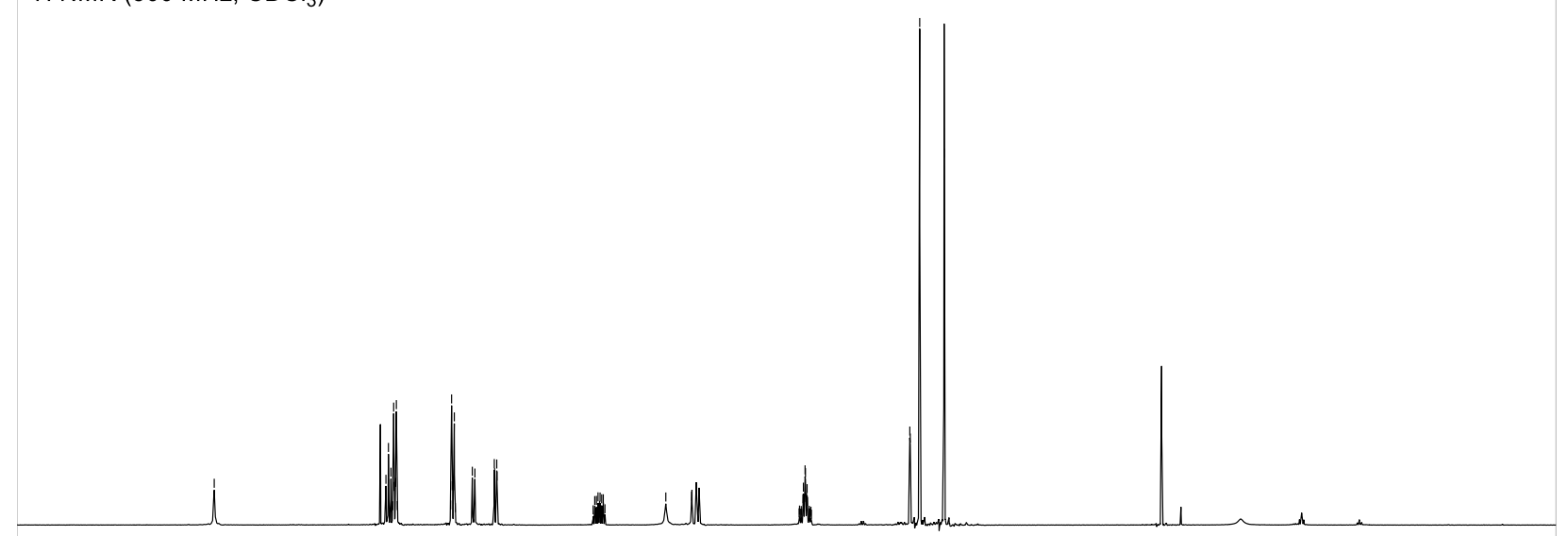

\begin{tabular}{|c|c|c|c|c|c|c|c|c|c|c|c|c|c|c|c|c|c|c|c|}
\hline & & $\begin{array}{l}\text { 'क } \\
\text { o } \\
0\end{array}$ & & & & 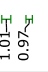 & 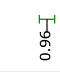 & $\begin{array}{l}\text { 'J' } \\
\text { o. } \\
0\end{array}$ & & 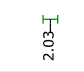 & & & & & & & & & \\
\hline 9.5 & 9.0 & 8.5 & 8.0 & 7.5 & 7.0 & 6.5 & 6.0 & 5.5 & 5.0 & $\begin{array}{r}4.5 \\
\text { f1 (ppm) }\end{array}$ & 4.0 & 3.5 & 3.0 & 2.5 & 2.0 & 1.5 & 1.0 & 0.5 & 0.0 \\
\hline
\end{tabular}

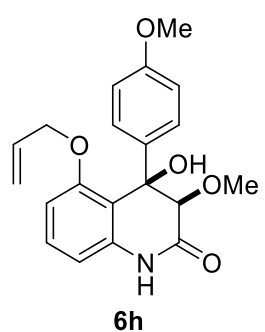

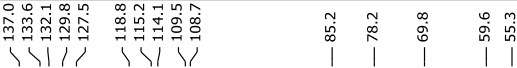

${ }^{13} \mathrm{C}$ NMR $\left(126 \mathrm{MHz}, \mathrm{CDCl}_{3}\right)$

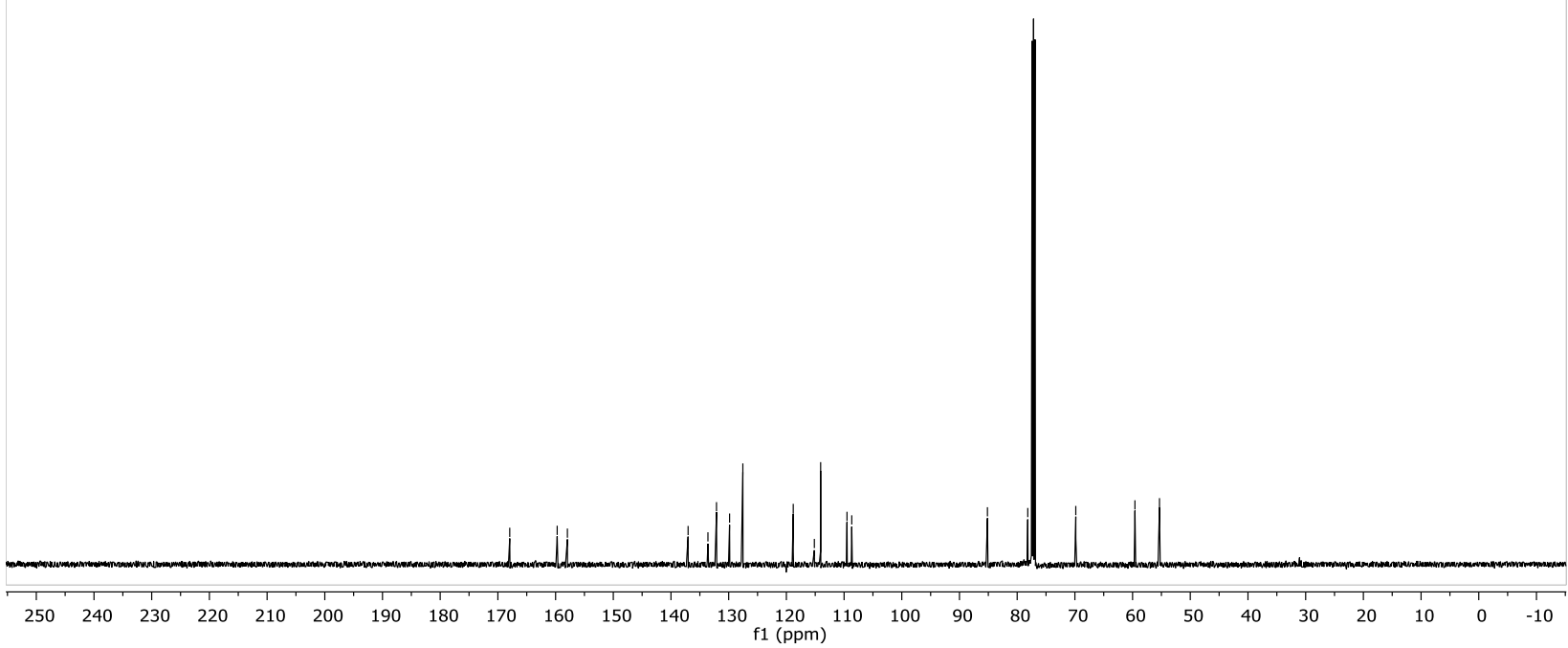




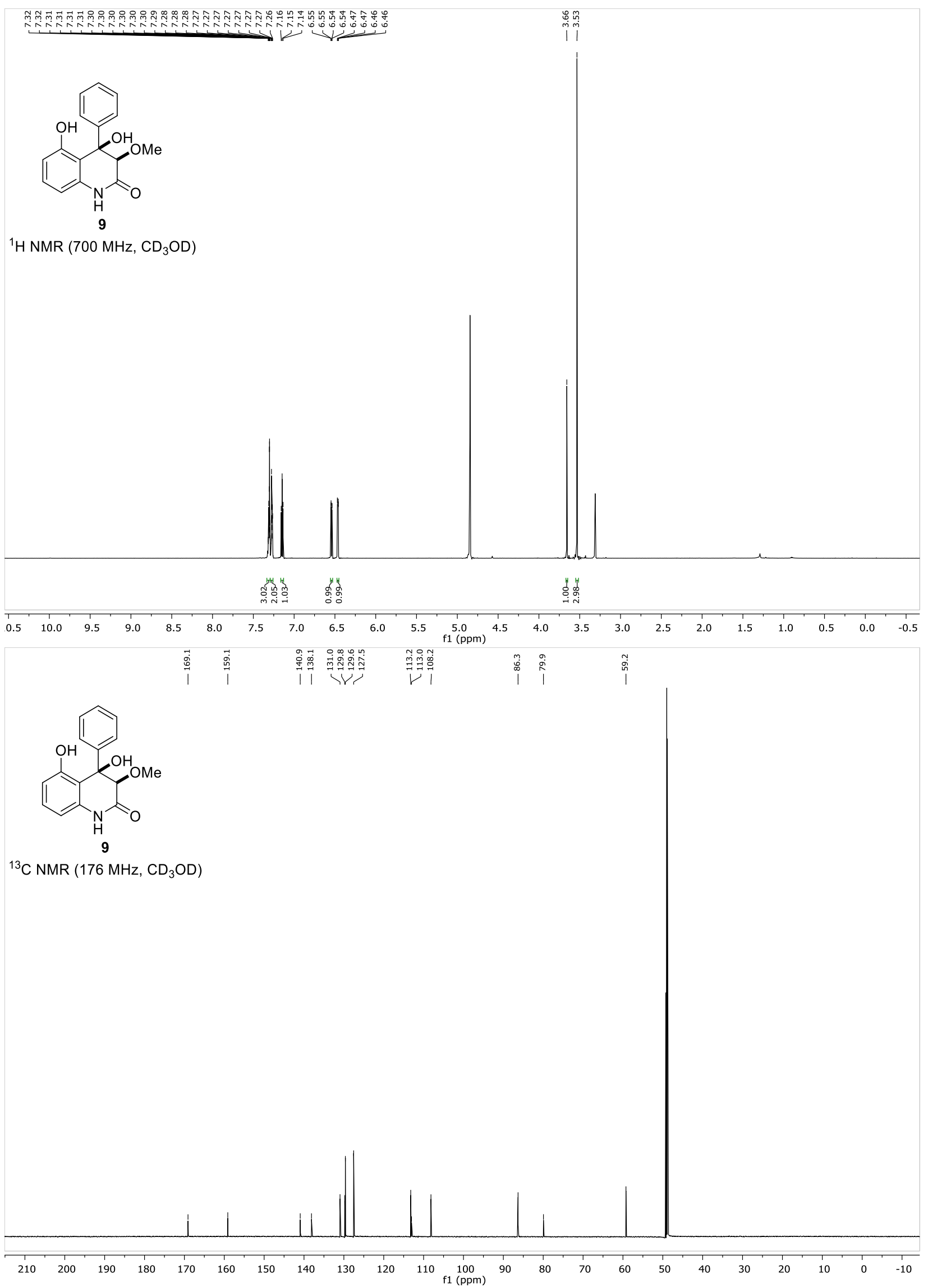




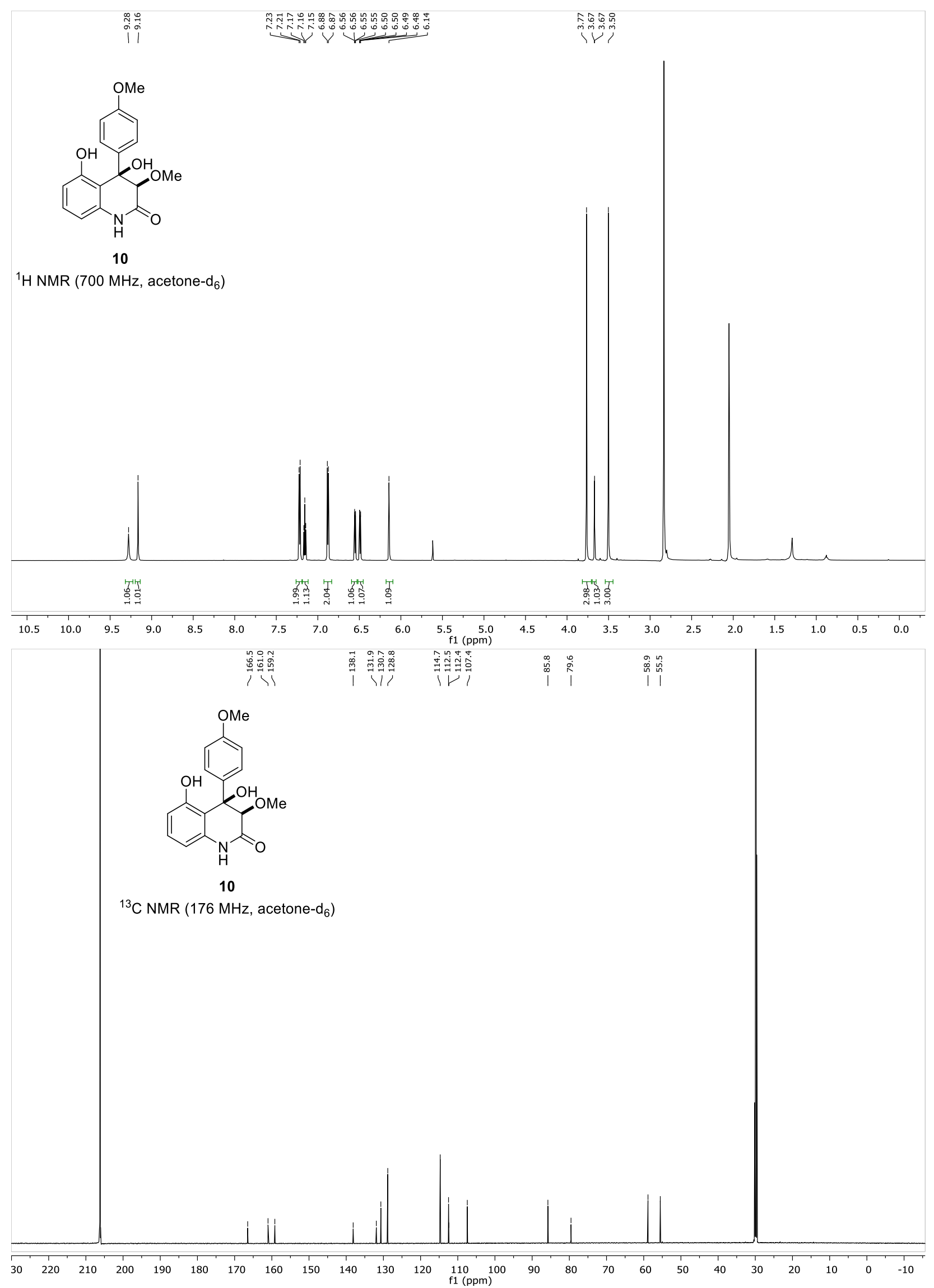




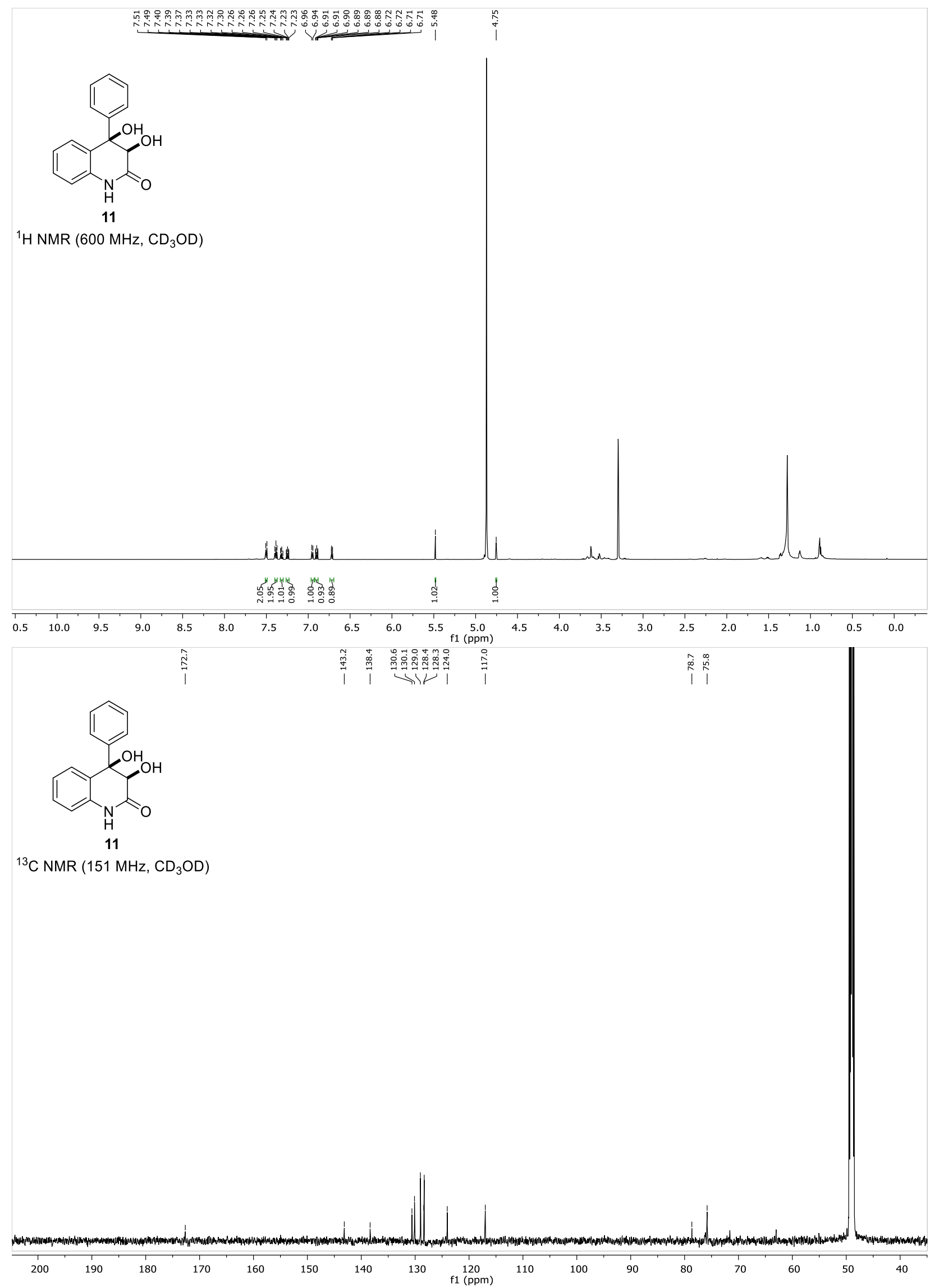


<smiles>C=CCc1ccc2c(c1O)[C@@](O)(c1ccccc1)C(OC)C(=O)N2</smiles>

$12 a$

${ }^{1} \mathrm{H}$ NMR $\left(500 \mathrm{MHz}, \mathrm{CDCl}_{3}\right)$
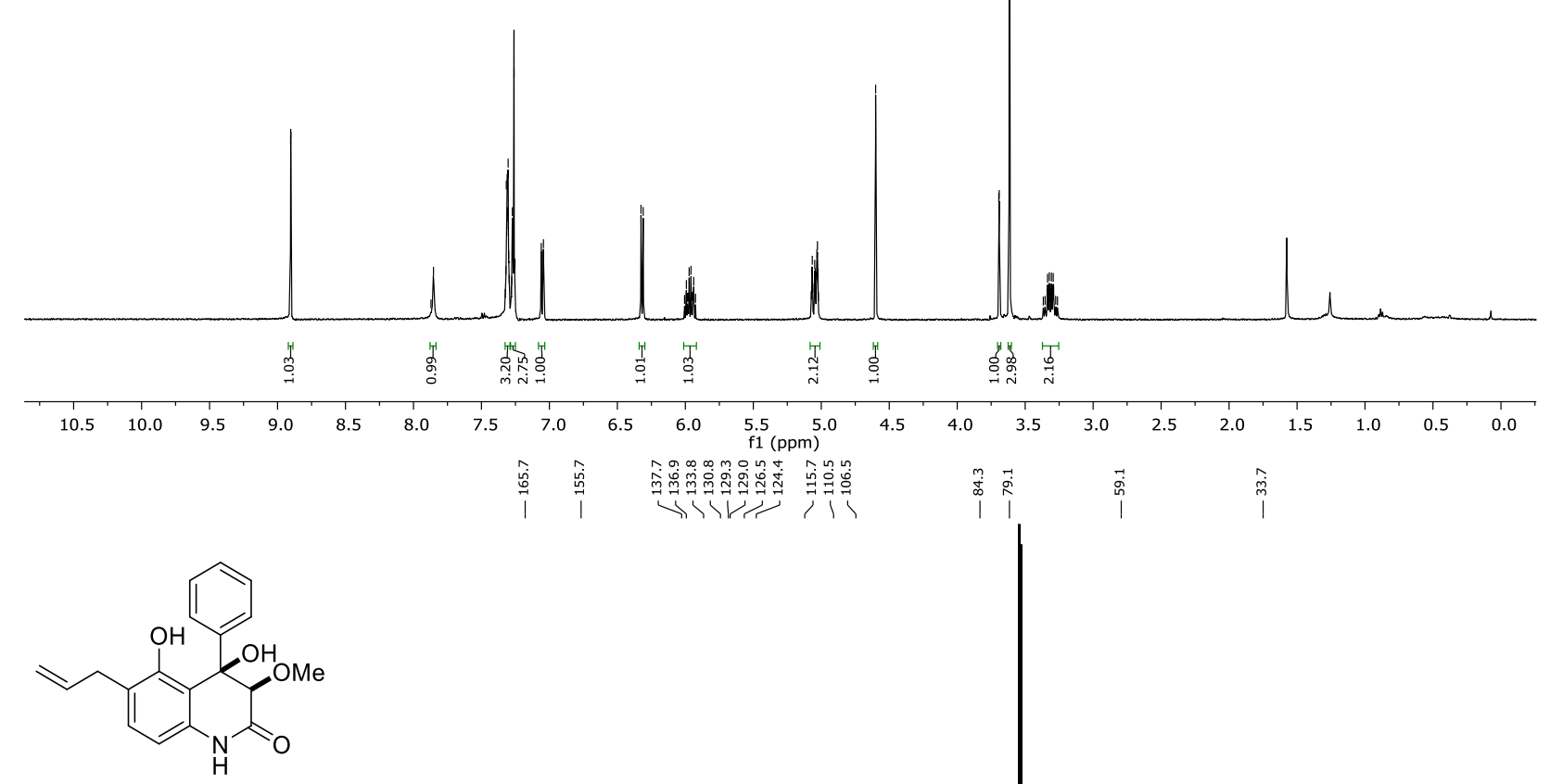

$12 \mathrm{a}$

${ }^{13} \mathrm{C}$ NMR $\left(126 \mathrm{MHz}, \mathrm{CDCl}_{3}\right)$

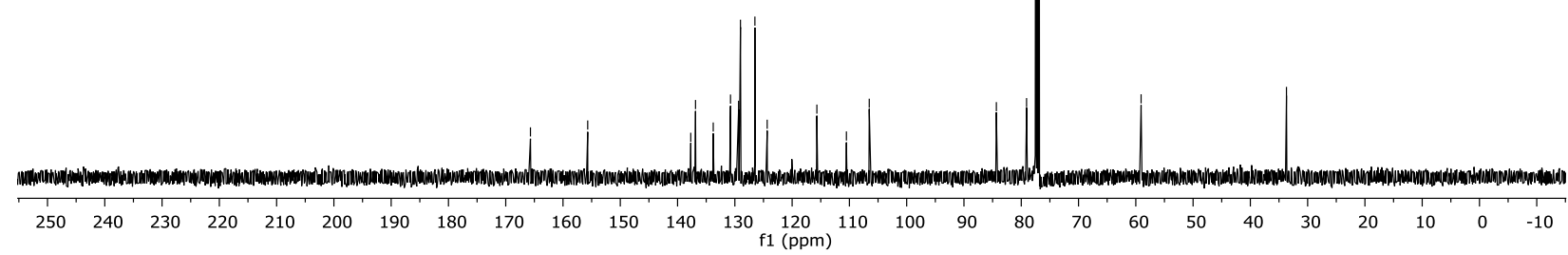


<smiles>C=CCc1ccc2c(c1O)[C@@](O)(c1ccc(OC)cc1)C(OC)C(=O)N2</smiles>

$12 b$

${ }^{1} \mathrm{H}$ NMR $\left(400 \mathrm{MHz}\right.$, acetone- $\left.\mathrm{d}_{6}\right)$

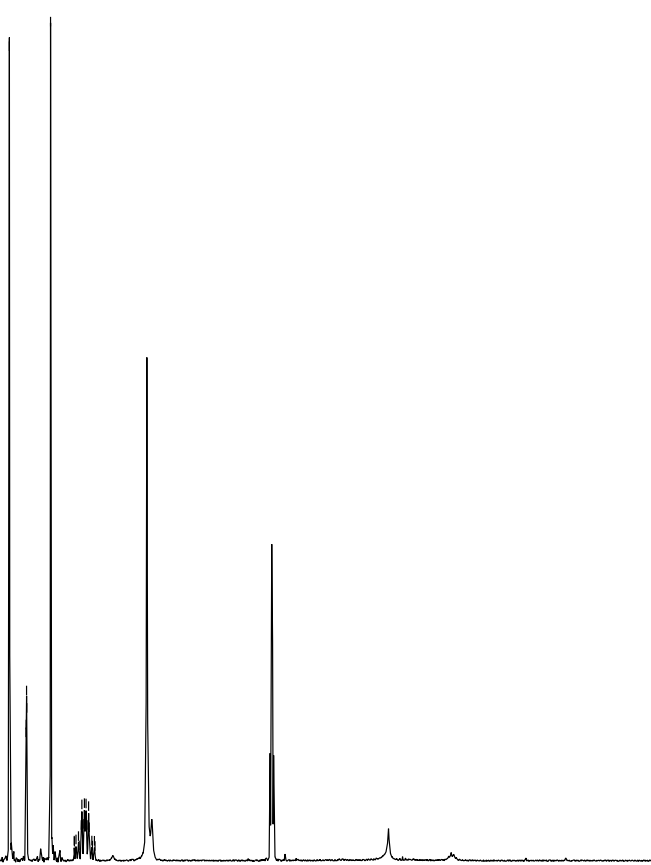

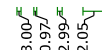

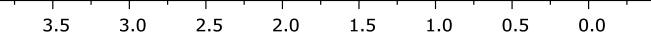
+o.

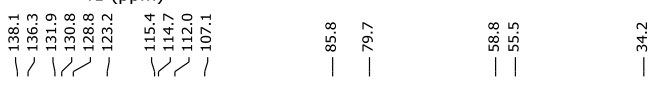<smiles>C=CCc1ccc2c(c1O)[C@@](O)(c1ccc(OC)cc1)C(OC)C(=O)N2</smiles>

$12 \mathrm{~b}$

${ }^{13} \mathrm{C}$ NMR (126 MHz, acetone- $\mathrm{d}_{6}$ )

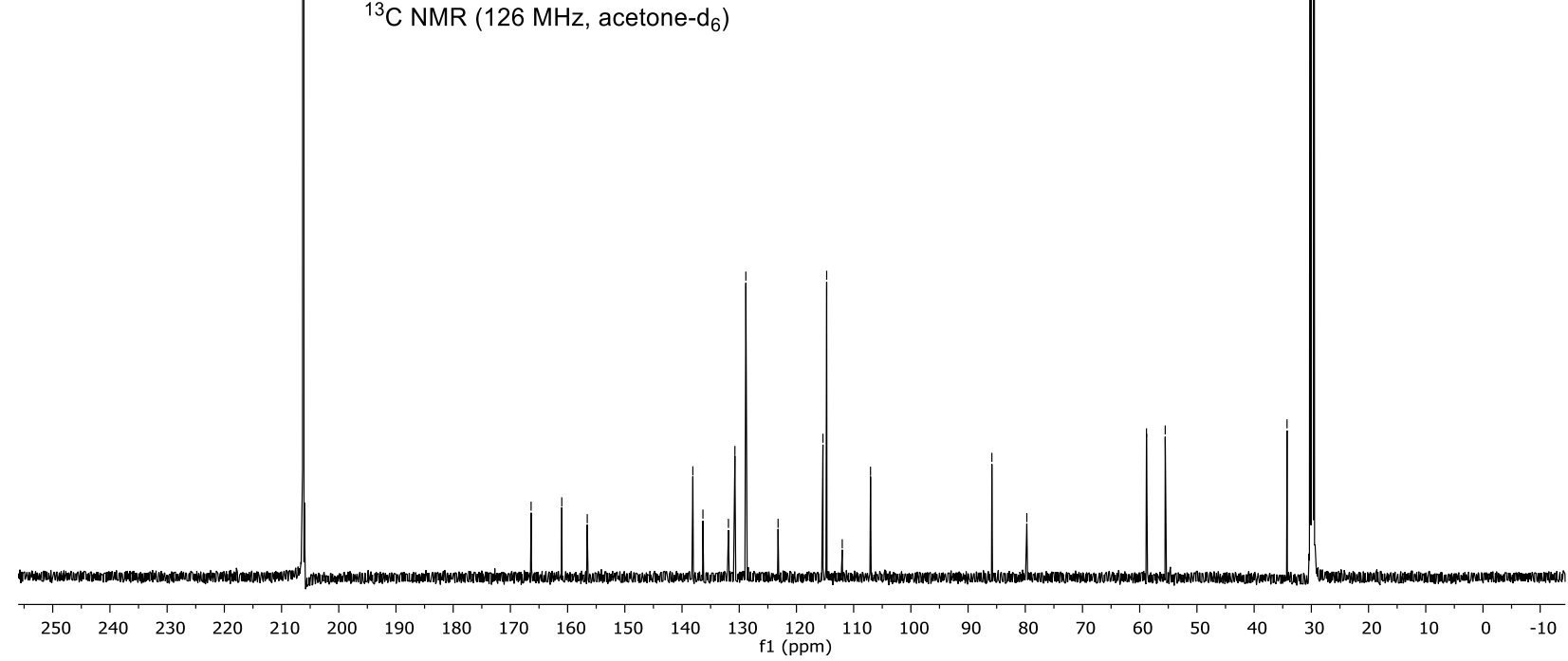




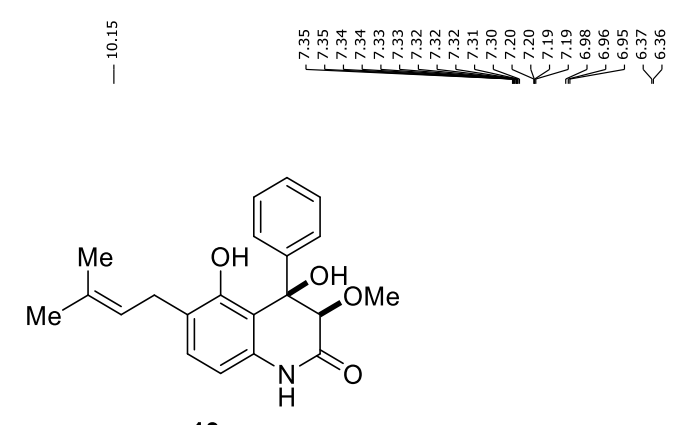

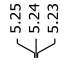

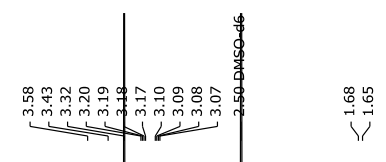

$13 a$

${ }^{1} \mathrm{H}$ NMR $\left(700 \mathrm{MHz}\right.$, DMSO-d $\left.\mathrm{d}_{6}\right)$

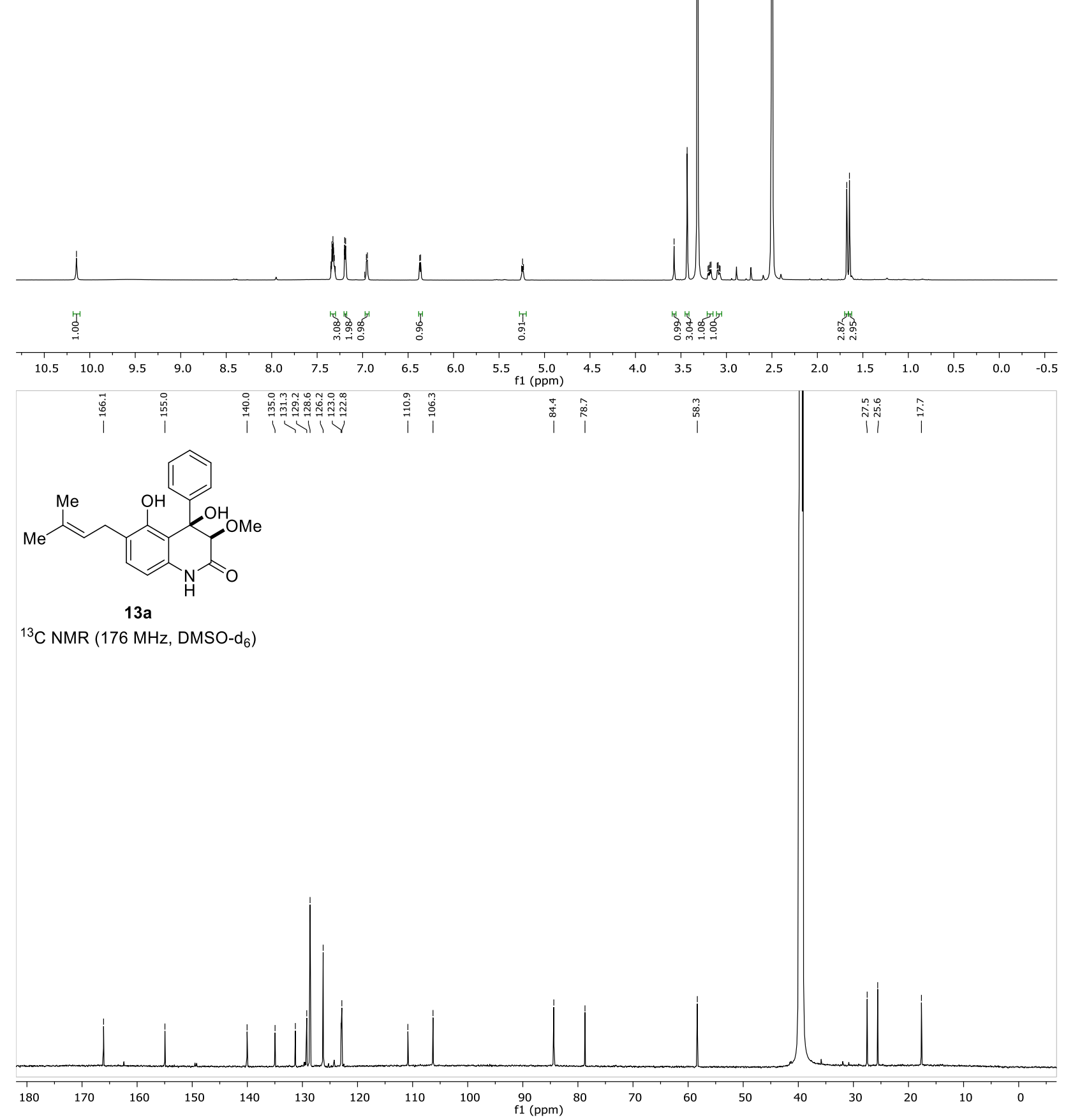



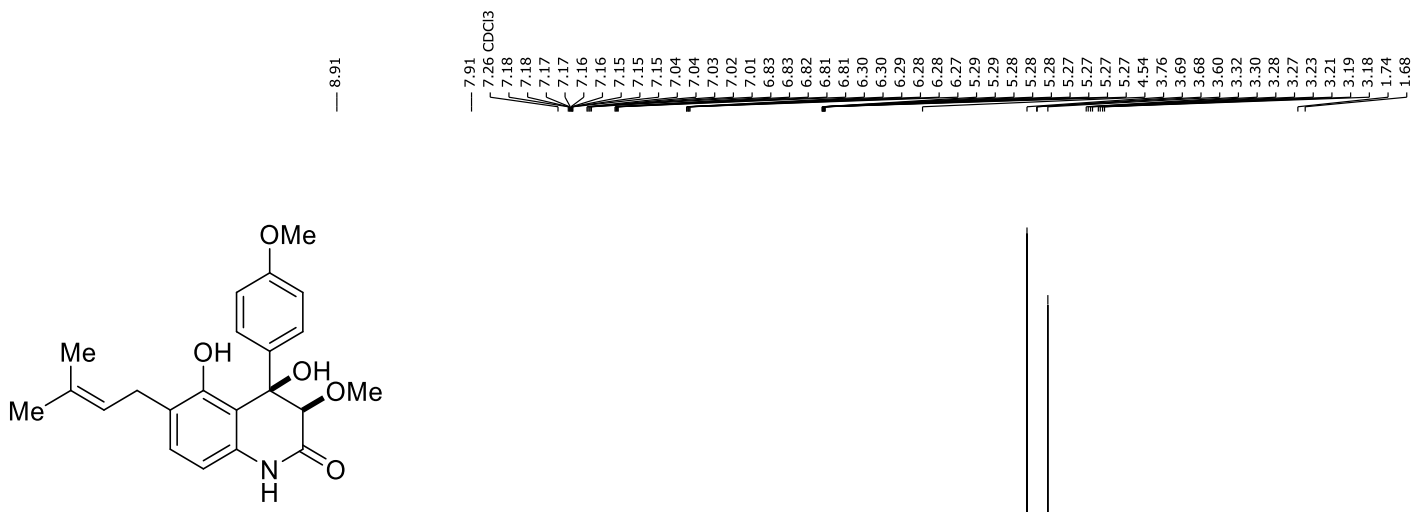

13b

${ }^{1} \mathrm{H}$ NMR $\left(500 \mathrm{MHz}, \mathrm{CDCl}_{3}\right)$
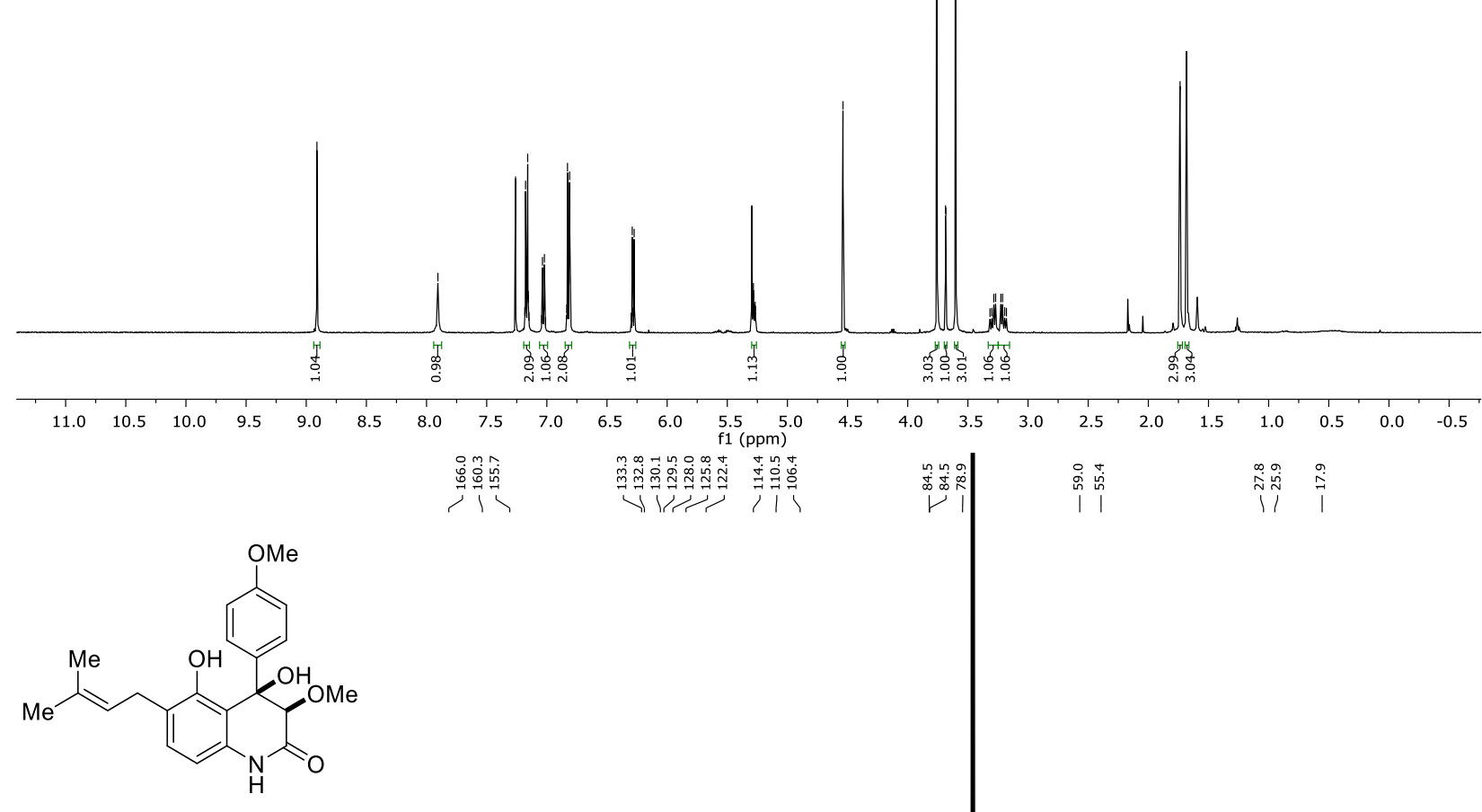

13b

${ }^{13} \mathrm{C}$ NMR $\left(126 \mathrm{MHz}, \mathrm{CDCl}_{3}\right)$

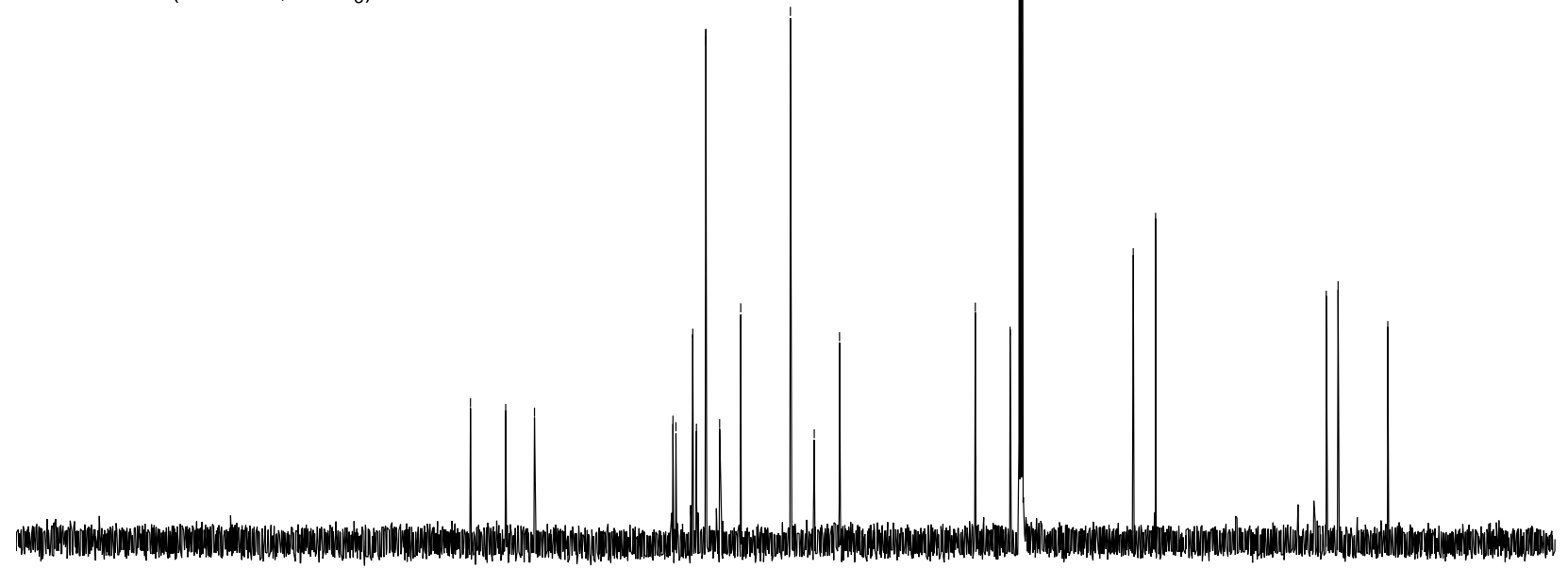

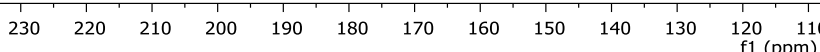

